\title{
Iodoimidazolinium-Catalyzed Reduction of Quinoline by Hantzsch Ester: Halogen Bond or Brønsted Acid Catalysis
}

Cher Tian Ser, Hui Yang and Ming Wah Wong*

Department of Chemistry, National University of Singapore, 3 Science Drive 3, Singapore 117543

\section{Supporting Information}

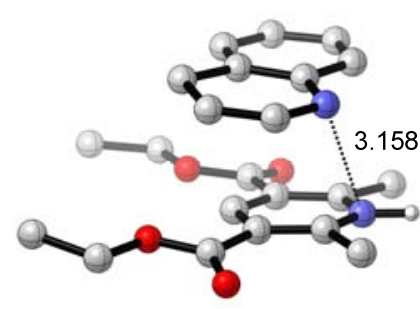

UN-INT1AE

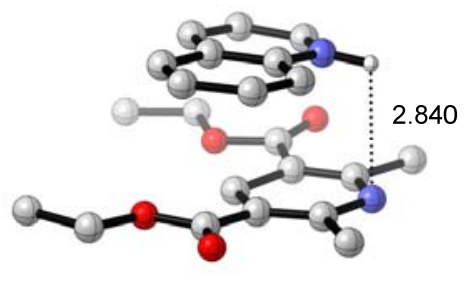

UN-INT1BD

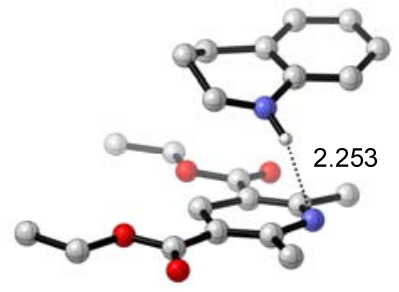

UN-INT2G

Figure S1. Optimized stacked geometries of various ion-pair intermediates. Interaction distances in $\AA$. 


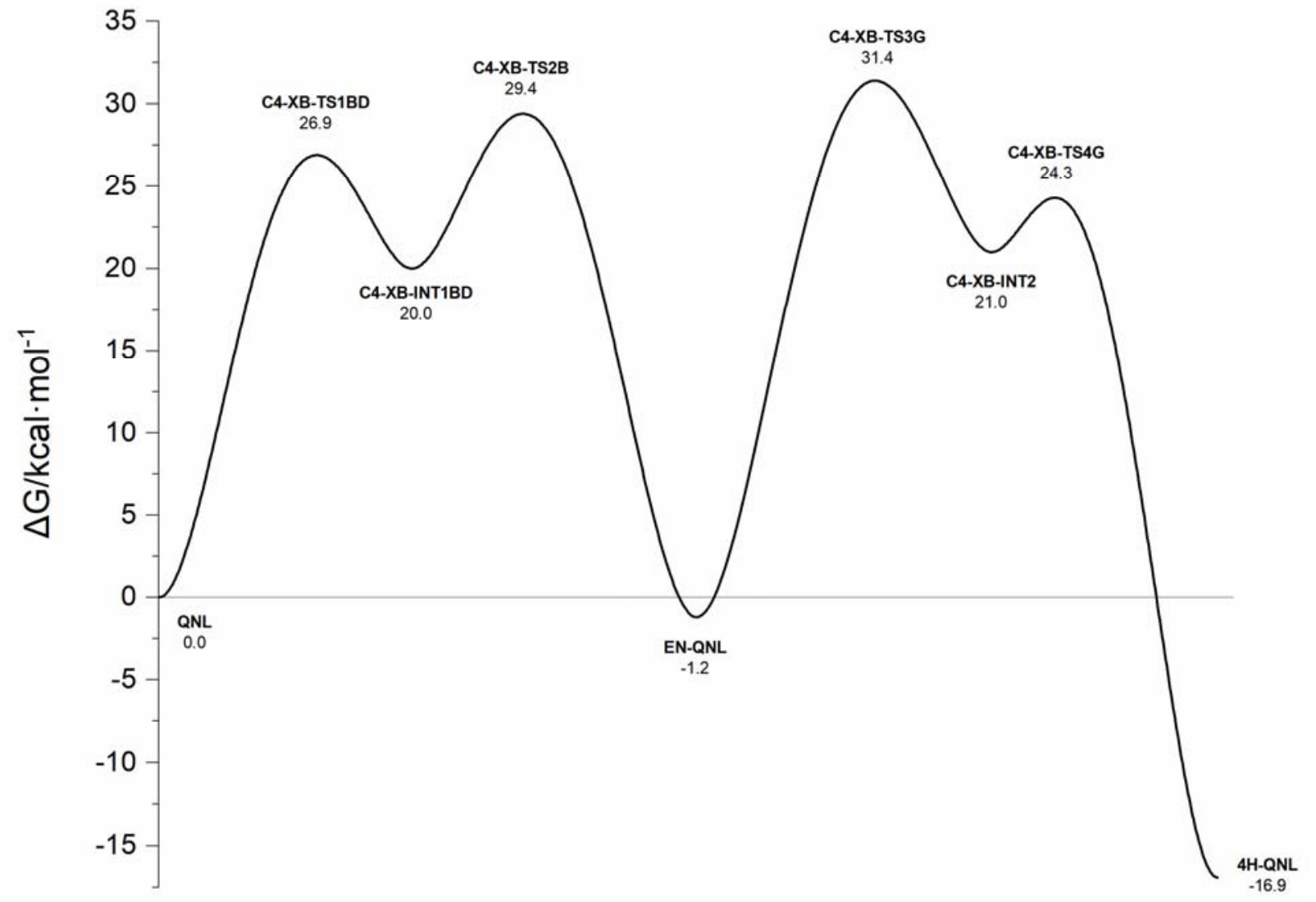

Figure S2. Schematic reaction profile of $\mathbf{C 4 - X B - c a t a l y z e d ~ r e a c t i o n . ~}$ 


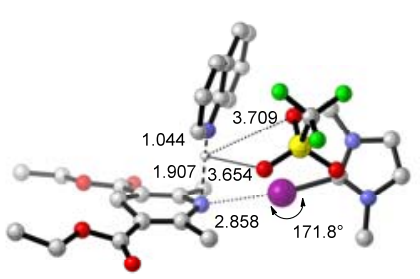

C4-XB-TS1BD

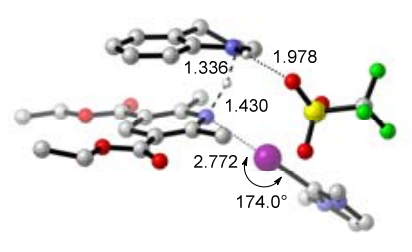

C4-XB-TS3G

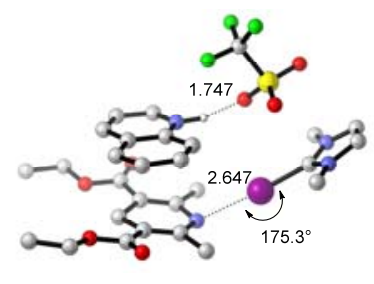

C4-XB-INT1BD

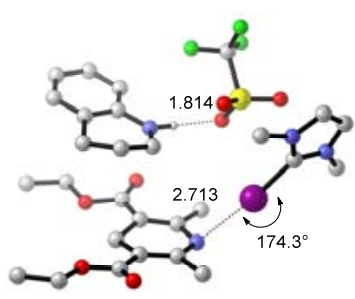

C4-XB-INT2G

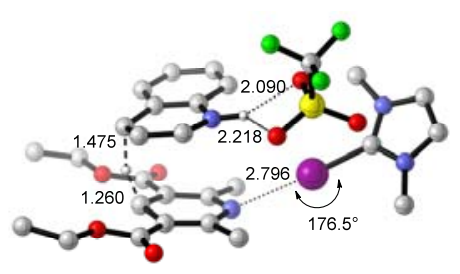

C4-XB-TS2B

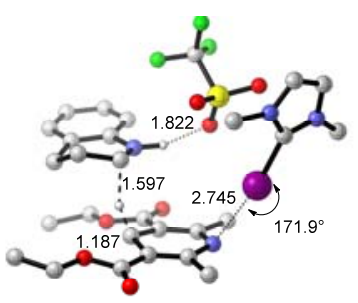

C4-XB-TS4G

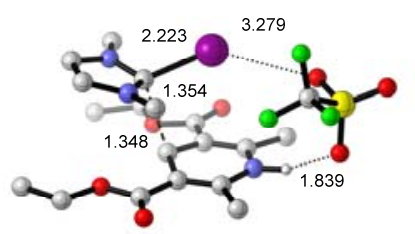

C4-BA-TS-GEN

Figure S3. Optimized geometries of XB- and BA-catalyzed reactions using $\mathbf{C 4}$ catalyst. 


\begin{tabular}{|c|c|c|c|c|}
\hline & 0.46944500 & 0.19391700 & 0.00544900 & \\
\hline $\mathrm{H}$ & 0.40022300 & 2.42682400 & 0.25384400 & \\
\hline $\mathrm{H}$ & -3.94457700 & 2.20067100 & $-0.0791410 c$ & \\
\hline $\mathrm{H}$ & -2.87321400 & 2.01840900 & -1.46711000 & \\
\hline H & -1.77168500 & 3.61030600 & 0.02370200 & \\
\hline & 2.90664700 & -0.00576200 & -0.06275500 & \\
\hline $\mathrm{H}$ & -2.06170100 & 2.59981500 & 1.43745000 & \\
\hline \multicolumn{3}{|c|}{ Zero-point correction $=$} & 0.1739 & 6 (Hartree/Particle) \\
\hline \multicolumn{3}{|c|}{ Thermal correction to Energy= } & & 3680 \\
\hline \multicolumn{3}{|c|}{ Thermal correction to Enthalpy= } & & 84624 \\
\hline \multicolumn{4}{|c|}{ Thermal correction to Gibbs Free Energy= } & 0.135565 \\
\hline \multicolumn{4}{|c|}{ Sum of electronic and zero-point Energies= } & -699.049041 \\
\hline \multicolumn{4}{|c|}{ Sum of electronic and thermal Energies $=$} & -699.039277 \\
\hline \multicolumn{4}{|c|}{ Sum of electronic and thermal Enthalpies= } & -699.038333 \\
\hline \multicolumn{4}{|c|}{ Sum of electronic and thermal Free Energies= } & -699.087392 \\
\hline
\end{tabular}

\section{H-QNL}

\begin{tabular}{|c|c|c|c|c|}
\hline & -1.13354300 & -1.37845400 & $-0.1642620 c$ & \\
\hline & -2.35846100 & -0.70695000 & 0.24680400 & \\
\hline $\mathrm{C}$ & -2.40103400 & 0.67433800 & -0.39571000 & \\
\hline $\mathrm{C}$ & -1.21366600 & 1.50491700 & 0.09285100 & \\
\hline $\mathrm{C}$ & 0.08414300 & 0.72499500 & 0.02652100 & \\
\hline $\mathrm{C}$ & 1.31525500 & 1.38044300 & 0.06334100 & \\
\hline $\mathrm{C}$ & 2.52236300 & 0.68737800 & 0.02912500 & \\
\hline $\mathrm{C}$ & 2.49742600 & -0.70516100 & -0.05215600 & \\
\hline $\mathrm{C}$ & 1.28591800 & -1.38184300 & -0.09881400 & \\
\hline $\mathrm{C}$ & 0.06838200 & -0.68222000 & -0.06180600 & \\
\hline $\mathrm{H}$ & -1.06869200 & -2.34700300 & 0.12806100 & \\
\hline $\mathrm{H}$ & -3.20550000 & -1.31649900 & -0.07931600 & \\
\hline $\mathrm{H}$ & -2.42018400 & -0.60142600 & 1.34221400 & \\
\hline $\mathrm{H}$ & -2.35674700 & 0.55552100 & -1.48445300 & \\
\hline $\mathrm{H}$ & -3.34313700 & 1.17328600 & -0.15045300 & \\
\hline $\mathrm{H}$ & -1.12034900 & 2.42558600 & -0.49360700 & \\
\hline $\mathrm{H}$ & -1.39705000 & 1.81604100 & 1.13008100 & \\
\hline $\mathrm{H}$ & 1.31798800 & 2.46721000 & 0.12464200 & \\
\hline $\mathrm{H}$ & 3.46542000 & 1.22365700 & 0.06195100 & \\
\hline $\mathrm{H}$ & 3.42538900 & -1.26942700 & -0.08012500 & \\
\hline $\mathrm{H}$ & 1.26388000 & -2.46708900 & -0.16818800 & \\
\hline \multicolumn{3}{|c|}{ Zero-point correction $=$} & 0.1843 & 0 (Hartree/Particle) \\
\hline \multicolumn{3}{|c|}{ Thermal correction to Energy= } & 0.1 & 2091 \\
\hline \multicolumn{3}{|c|}{ Thermal correction to Enthalpy= } & & 93035 \\
\hline \multicolumn{4}{|c|}{ Thermal correction to Gibbs Free Energy= } & 0.152022 \\
\hline \multirow{2}{*}{\multicolumn{4}{|c|}{$\begin{array}{l}\text { Sum of electronic and zero-point Energies= } \\
\text { Sum of electronic and thermal Energies }=\end{array}$}} & -403.987648 \\
\hline & & & & -403.979867 \\
\hline \multicolumn{4}{|c|}{ Sum of electronic and thermal Enthalpies= } & -403.978923 \\
\hline \multicolumn{5}{|c|}{ Sum of electronic and thermal Free Energies= } \\
\hline
\end{tabular}




\begin{tabular}{|c|c|c|c|c|}
\hline \multicolumn{5}{|c|}{ AM-QNL } \\
\hline \multicolumn{5}{|c|}{$\begin{array}{ll}\mathrm{N} & 1.18423900\end{array}$} \\
\hline $\mathrm{C}$ & 2.41973700 & -0.77126800 & 0.16267500 & \\
\hline $\mathrm{C}$ & 2.41632200 & 0.71659400 & -0.07381600 & \\
\hline $\mathrm{C}$ & 1.26828900 & 1.39609300 & -0.15551900 & \\
\hline $\mathrm{C}$ & -0.02171800 & 0.70858100 & -0.05922600 & \\
\hline $\mathrm{C}$ & -1.23457200 & 1.38534600 & 0.06954200 & \\
\hline $\mathrm{C}$ & -2.44336900 & 0.69518800 & 0.13078700 & \\
\hline $\mathrm{C}$ & -2.43370200 & -0.69709500 & 0.05008600 & \\
\hline $\mathrm{C}$ & -1.23563800 & -1.39417200 & -0.08213000 & \\
\hline $\mathrm{C}$ & -0.01962700 & -0.70304200 & -0.13564800 & \\
\hline $\mathrm{H}$ & 3.37578900 & 1.22277500 & -0.12625400 & \\
\hline $\mathrm{H}$ & 1.26246500 & 2.47536000 & -0.28961800 & \\
\hline $\mathrm{H}$ & -1.22087400 & 2.47180700 & 0.12134800 & \\
\hline $\mathrm{H}$ & -3.37893200 & 1.23496100 & 0.23517700 & \\
\hline $\mathrm{H}$ & -3.36710300 & -1.25117500 & 0.09389600 & \\
\hline $\mathrm{H}$ & -1.23223800 & -2.47960900 & -0.14646900 & \\
\hline $\mathrm{H}$ & 3.25896600 & -1.23909100 & -0.36054000 & \\
\hline $\mathrm{H}$ & 2.56358800 & -0.97264100 & 1.23981600 & \\
\hline $\mathrm{H}$ & 1.13066200 & -2.36400300 & -0.22323700 & \\
\hline \multicolumn{5}{|c|}{ Zero-point correction $=$} \\
\hline \multicolumn{3}{|c|}{ Thermal correction to Energy= } & 0.16 & 7772 \\
\hline \multicolumn{3}{|c|}{ Thermal correction to Enthalpy= } & 0.1 & 8716 \\
\hline \multicolumn{4}{|c|}{ Thermal correction to Gibbs Free Energy= } & 0.128346 \\
\hline \multicolumn{4}{|c|}{ Sum of electronic and zero-point Energies= } & -402.790792 \\
\hline \multicolumn{4}{|c|}{ Sum of electronic and thermal Energies $=$} & -402.783340 \\
\hline \multicolumn{4}{|c|}{ Sum of electronic and thermal Enthalpies $=$} & -402.782396 \\
\hline \multicolumn{4}{|c|}{ Sum of electronic and thermal Free Energies $=$} & -402.822766 \\
\hline \multicolumn{5}{|c|}{ BA-CAT } \\
\hline $\mathrm{C}$ & -3.94127000 & 3.49233100 & 0.01707300 & \\
\hline $\mathrm{C}$ & -3.94235200 & -3.49174100 & 0.02702000 & \\
\hline $\mathrm{C}$ & 1.23446700 & 2.40597800 & -0.04858200 & \\
\hline $\mathrm{C}$ & 1.23423900 & -2.40693900 & -0.04427500 & \\
\hline $\mathrm{C}$ & -5.37898800 & 3.03695400 & -0.06068800 & \\
\hline $\mathrm{C}$ & -5.37998100 & -3.03580100 & -0.04910200 & \\
\hline $\mathrm{H}$ & 1.99232600 & -0.00045600 & -0.08997900 & \\
\hline 0 & -1.28640800 & 3.59880400 & 0.11910300 & \\
\hline 0 & -1.28734600 & -3.59917900 & 0.12544000 & \\
\hline $\mathrm{C}$ & -1.72874900 & -0.00019500 & 0.05005700 & \\
\hline $\mathrm{C}$ & -1.80458600 & 2.50831400 & 0.05360500 & \\
\hline $\mathrm{C}$ & -1.80516000 & -2.50856800 & 0.05887800 & \\
\hline 0 & -3.11703400 & 2.30633000 & -0.00576300 & \\
\hline 0 & -3.11762100 & -2.30614800 & 0.00106500 & \\
\hline $\mathrm{C}$ & -1.04960200 & 1.21528100 & 0.02868700 & \\
\hline $\mathrm{C}$ & 0.34625900 & 1.20703600 & -0.01955800 & \\
\hline
\end{tabular}




\begin{tabular}{|c|c|c|c|c|}
\hline $\mathrm{C}$ & -1.04979500 & -1.21579700 & 0.03119700 & \\
\hline $\mathrm{C}$ & 0.34611700 & -1.20784800 & -0.01721000 & \\
\hline $\mathrm{N}$ & 0.95176300 & -0.00045200 & -0.04268400 & \\
\hline $\mathrm{H}$ & -2.81174500 & -0.00006100 & 0.08512000 & \\
\hline $\mathrm{H}$ & 1.08786100 & 2.99555600 & 0.85988600 & \\
\hline $\mathrm{H}$ & 2.28087600 & 2.10448900 & -0.12739600 & \\
\hline $\mathrm{H}$ & 0.96859800 & 3.04625400 & -0.89279700 & \\
\hline $\mathrm{H}$ & -3.66123400 & 4.12205300 & -0.83156100 & \\
\hline $\mathrm{H}$ & -3.73024600 & 4.04067900 & 0.93902700 & \\
\hline $\mathrm{H}$ & 0.96837500 & -3.04845200 & -0.88755400 & \\
\hline $\mathrm{H}$ & 2.28069500 & -2.10571700 & -0.12352100 & \\
\hline $\mathrm{H}$ & 1.08751900 & -2.99515400 & 0.86504600 & \\
\hline $\mathrm{H}$ & -5.56037000 & 2.48122700 & -0.98476900 & \\
\hline $\mathrm{H}$ & -5.63091200 & 2.39918100 & 0.79104400 & \\
\hline $\mathrm{H}$ & -6.03677400 & 3.91018800 & -0.04661500 & \\
\hline $\mathrm{H}$ & -3.73011600 & -4.03878500 & 0.94947200 & \\
\hline $\mathrm{H}$ & -3.66396900 & -4.12288300 & -0.82110000 & \\
\hline $\mathrm{H}$ & -5.63020100 & -2.39661600 & 0.80207400 & \\
\hline $\mathrm{H}$ & -5.56260100 & -2.48137900 & -0.97372300 & \\
\hline $\mathrm{H}$ & -6.03816600 & -3.90869100 & -0.03264100 & \\
\hline & 4.49850700 & 0.00043100 & -0.28320200 & \\
\hline \multicolumn{5}{|c|}{ Zero-point correction $=$} \\
\hline \multicolumn{3}{|c|}{ Thermal correction to Energy= } & 0.32 & 4791 \\
\hline \multicolumn{3}{|c|}{ Thermal correction to Enthalpy= } & 0.3 & 25735 \\
\hline \multicolumn{4}{|c|}{ Thermal correction to Gibbs Free Energy= } & 0.250911 \\
\hline \multicolumn{4}{|c|}{ Sum of electronic and zero-point Energies= } & -1156.919261 \\
\hline \multicolumn{4}{|c|}{ Sum of electronic and thermal Energies $=$} & -1156.898748 \\
\hline \multicolumn{4}{|c|}{ Sum of electronic and thermal Enthalpies= } & -1156.897804 \\
\hline \multicolumn{4}{|c|}{ Sum of electronic and thermal Free Energies= } & -1156.972627 \\
\hline
\end{tabular}

\begin{tabular}{lccc}
\multicolumn{4}{l}{ BA-TS1BD } \\
C & 4.24451900 & -3.64988700 & 0.94939400 \\
$\mathrm{C}$ & 4.37540600 & 2.33461300 & -2.70521400 \\
$\mathrm{C}$ & -0.85435400 & -2.34291000 & 0.78812900 \\
$\mathrm{C}$ & -0.74473800 & 1.81086700 & -1.63496600 \\
$\mathrm{C}$ & 5.69885700 & -3.29609700 & 0.74537100 \\
$\mathrm{C}$ & 5.70311700 & 1.67648000 & -2.99810200 \\
$\mathrm{H}$ & -1.82486200 & -0.13165000 & -0.27346700 \\
0 & 1.59568900 & -3.66013300 & 0.99466000 \\
0 & 1.87558500 & 2.85031000 & -1.98827600 \\
$\mathrm{C}$ & 2.13791300 & -0.49361000 & -0.68099900 \\
$\mathrm{C}$ & 2.14049800 & -2.68443300 & 0.52882400 \\
$\mathrm{C}$ & 2.28018600 & 1.72049800 & -1.83003700 \\
0 & 3.46373300 & -2.54784200 & 0.44558700 \\
0 & 3.50432900 & 1.31742100 & -2.17045100 \\
$\mathrm{C}$ & 1.42649700 & -1.49776500 & -0.03176900
\end{tabular}




\begin{tabular}{|c|c|c|c|}
\hline $\mathrm{C}$ & 0.03542300 & -1.36507600 & 0.08175400 \\
\hline $\mathrm{C}$ & 1.48784900 & 0.61569700 & -1.21133400 \\
\hline $\mathrm{C}$ & 0.09485700 & 0.69103500 & -1.10098400 \\
\hline $\mathrm{N}$ & -0.56319500 & -0.29513400 & -0.46703300 \\
\hline $\mathrm{H}$ & 3.21540200 & -0.57108000 & -0.76467900 \\
\hline $\mathrm{H}$ & -0.49848400 & -2.50554800 & 1.80823500 \\
\hline $\mathrm{H}$ & -1.88060600 & -1.97540200 & 0.82013400 \\
\hline $\mathrm{H}$ & -0.83092100 & -3.31245700 & 0.28428300 \\
\hline $\mathrm{H}$ & 3.96049900 & -4.55473600 & 0.40477300 \\
\hline $\mathrm{H}$ & 4.00014300 & -3.79328400 & 2.00544700 \\
\hline $\mathrm{H}$ & -0.34055900 & 2.19542800 & -2.57058600 \\
\hline $\mathrm{H}$ & -1.77166300 & 1.47523100 & -1.79185200 \\
\hline $\mathrm{H}$ & -0.74715200 & 2.63527100 & -0.91201300 \\
\hline $\mathrm{H}$ & 5.91582600 & -3.14536300 & -0.31582900 \\
\hline $\mathrm{H}$ & 5.95560300 & -2.38390900 & 1.29083400 \\
\hline $\mathrm{H}$ & 6.32726800 & -4.11064000 & 1.11584000 \\
\hline $\mathrm{H}$ & 4.46667300 & 3.13584300 & -1.96670300 \\
\hline $\mathrm{H}$ & 3.91325900 & 2.74833700 & -3.60571900 \\
\hline $\mathrm{H}$ & 6.13878300 & 1.26088800 & -2.08528800 \\
\hline $\mathrm{H}$ & 5.58467100 & 0.87275500 & -3.73014300 \\
\hline $\mathrm{H}$ & 6.39519600 & 2.41820000 & -3.40604400 \\
\hline $\mathrm{C}$ & -6.46827100 & -0.79343100 & -1.10549200 \\
\hline $\mathrm{C}$ & -6.16669100 & -1.66641400 & -2.12013700 \\
\hline $\mathrm{C}$ & -4.81706600 & -1.95817900 & -2.43963200 \\
\hline $\mathrm{C}$ & -3.78646400 & -1.37552500 & -1.74419400 \\
\hline $\mathrm{C}$ & -4.08268600 & -0.47240100 & -0.69541100 \\
\hline $\mathrm{C}$ & -5.42876000 & -0.17366000 & -0.36552500 \\
\hline $\mathrm{H}$ & -6.96227600 & -2.14058200 & -2.68524200 \\
\hline $\mathrm{H}$ & -4.59845500 & -2.65177000 & -3.24512900 \\
\hline $\mathrm{H}$ & -2.74902200 & -1.59355500 & -1.98111000 \\
\hline $\mathrm{H}$ & -7.49867900 & -0.56280100 & -0.85143300 \\
\hline $\mathrm{C}$ & -5.66966200 & 0.73559200 & 0.69579400 \\
\hline $\mathrm{C}$ & -4.61703900 & 1.29912600 & 1.37181900 \\
\hline $\mathrm{C}$ & -3.30494900 & 0.95633300 & 0.98862400 \\
\hline $\mathrm{N}$ & -3.06313800 & 0.11309400 & 0.00375000 \\
\hline $\mathrm{H}$ & -6.69602400 & 0.97446000 & 0.96057200 \\
\hline $\mathrm{H}$ & -4.76781500 & 1.99617200 & 2.18723800 \\
\hline $\mathrm{H}$ & -2.42969400 & 1.37033000 & 1.48999000 \\
\hline I & 0.30925400 & 1.91710000 & 2.42387200 \\
\hline \multicolumn{4}{|r|}{$0.438300($ Hartree/Particle $)$} \\
\hline \multicolumn{3}{|c|}{ Thermal correction to Energy= } & 0.468134 \\
\hline \multicolumn{3}{|c|}{ Thermal correction to Enthalpy= } & 0.469078 \\
\hline \multicolumn{4}{|c|}{ Thermal correction to Gibbs Free Energy $=\quad 0.370380$} \\
\hline \multicolumn{4}{|c|}{ Sum of electronic and zero-point Energies $=$} \\
\hline \multicolumn{4}{|c|}{ Sum of electronic and thermal Energies $=$} \\
\hline & $\mathrm{n}$ of electronic & and thermal En & -1558.531052 \\
\hline
\end{tabular}




\section{BA-TS2B}

$\begin{array}{cccc}\mathrm{C} & 3.83725200 & -3.08072800 & -0.90606600 \\ \mathrm{C} & 3.65335600 & 3.86468800 & 0.32049300 \\ \mathrm{C} & -1.16670400 & -1.88090000 & -1.95626200 \\ \mathrm{C} & -1.28982300 & 2.84099700 & -1.09221900 \\ \mathrm{C} & 5.14921400 & -2.73314700 & -0.24168300 \\ \mathrm{C} & 4.95131400 & 3.36762500 & 0.91349400 \\ \mathrm{H} & -1.98679400 & 0.44371400 & -1.42202700 \\ \mathrm{O} & 1.42084600 & -3.03471300 & -1.97680400 \\ \mathrm{O} & 1.23569600 & 4.06679200 & -0.74120600 \\ \mathrm{C} & 1.73079900 & 0.43948000 & -0.85391500 \\ \mathrm{C} & 1.81320500 & -2.01238700 & -1.45536600 \\ \mathrm{C} & 1.67911300 & 2.94805400 & -0.58496100 \\ 0 & 3.02805900 & -1.89286400 & -0.89952200 \\ \mathrm{O} & 2.88972700 & 2.70635000 & -0.05978700 \\ \mathrm{C} & 1.05125100 & -0.74962700 & -1.32786900 \\ \mathrm{C} & -0.30736100 & -0.72881700 & -1.53283700 \\ \mathrm{C} & 0.99047700 & 1.68291700 & -0.91576800 \\ \mathrm{C} & -0.36854000 & 1.66078300 & -1.11920500 \\ \mathrm{~N} & -0.96379700 & 0.45906300 & -1.36471200 \\ \mathrm{H} & 1.87076800 & 0.24809500 & 0.48722200 \\ \mathrm{H} & 2.80060200 & 0.49691500 & -1.04110500 \\ \mathrm{H} & -0.87397600 & -2.79514200 & -1.44217700 \\ \mathrm{H} & -2.21823900 & -1.66058100 & -1.75049800 \\ \mathrm{H} & -1.04746800 & -2.05395400 & -3.03100100 \\ \mathrm{H} & 3.97564800 & -3.40834600 & -1.94025500 \\ \mathrm{H} & 3.29994400 & -3.86940300 & -0.36924600 \\ \mathrm{H} & -1.16042900 & 3.43678500 & -2.00092800 \\ \mathrm{H} & -2.32935800 & 2.50809700 & -1.03110600 \\ \mathrm{H} & -1.06062500 & 3.49080300 & -0.24689900 \\ \mathrm{H} & 5.66161100 & -1.93800700 & -0.79058800 \\ \mathrm{H} & 4.98910100 & -2.39974400 & 0.78802300 \\ \mathrm{H} & 5.79614900 & -3.61447400 & -0.22340400 \\ \mathrm{H} & 3.07094000 & 4.44751800 & 1.04010600 \\ \mathrm{H} & 3.81653500 & 4.48379600 & -0.56638300 \\ \mathrm{H} & 4.76094600 & 2.74462600 & 1.79242000 \\ \mathrm{H} & 5.51196500 & 2.77997500 & 0.18114900 \\ \mathrm{H} & 5.56548300 & 4.21924100 & 1.21859500 \\ \mathrm{~N} & -1.22156300 & 0.20438200 & 1.67715200 \\ & -0.51862600 & 1.32061100 & 1.98846700 \\ \mathrm{C} & 1.56210400 & 0.09414000 & 1.78854000 \\ & & \end{array}$




\begin{tabular}{|c|c|c|c|}
\hline & & & \\
\hline & 79877600 & & \\
\hline & -1.41297200 & & \\
\hline & -0.62873600 & & \\
\hline & -1.11011800 & 2.21704600 & \\
\hline & & & \\
\hline & & & \\
\hline & & & \\
\hline & & & \\
\hline & & & \\
\hline & & & \\
\hline & -2.2 & & \\
\hline & -4.3907 & 0.23 & \\
\hline \multicolumn{3}{|c|}{ Zero-point correction $=$} & \\
\hline \multicolumn{3}{|c|}{ Thermal correction to Energy= } & \\
\hline \multicolumn{4}{|c|}{ Thermal correction to Enthalpy= } \\
\hline \multicolumn{4}{|c|}{ Thermal correction to Gibbs Free Energy= } \\
\hline \multicolumn{4}{|c|}{ Sum of electronic and zero-point Energies $=$} \\
\hline \multicolumn{4}{|c|}{ Sum of electronic and thermal Energies= } \\
\hline \multirow{2}{*}{\multicolumn{4}{|c|}{$\begin{array}{l}\text { Sum of electronic and thermal Enthalpies= } \\
\text { Sum of electronic and thermal Free Energies= }\end{array}$}} \\
\hline & & & \\
\hline \multicolumn{4}{|c|}{ BA-TS3G } \\
\hline & -2.67277 & & \\
\hline & & & \\
\hline & & & \\
\hline & & & \\
\hline & & & \\
\hline & & & \\
\hline & & & \\
\hline & & & \\
\hline & & & \\
\hline & & & \\
\hline & & & \\
\hline & & & \\
\hline & & & \\
\hline & & & \\
\hline & -0.2 & & \\
\hline & & & \\
\hline & & & \\
\hline & & & \\
\hline & & & \\
\hline & & & \\
\hline & 0.93087800 & & 0.6994050 \\
\hline & & & \\
\hline & & & \\
\hline
\end{tabular}




\begin{tabular}{|c|c|c|c|c|}
\hline \multirow{2}{*}{$\begin{array}{l}\mathrm{H} \\
\mathrm{H}\end{array}$} & & -1.10296200 & 3.45238000 & \\
\hline & -2.70345900 & 3.72461400 & 1.73577200 & \\
\hline $\mathrm{H}$ & $\begin{array}{l}-2.70345900 \\
-3.54889200\end{array}$ & 2.53389000 & 0.71816400 & \\
\hline $\mathrm{I}$ & 3.97155800 & -1.39274400 & 2.45284500 & \\
\hline $\mathrm{H}$ & 3.01368300 & -2.80781200 & 1.97007200 & \\
\hline $\mathrm{H}$ & \multirow{2}{*}{3.91923200} & -1.84192400 & 0.75743400 & \\
\hline $\mathrm{H}$ & & 4.72823300 & -0.34017500 & \\
\hline $\mathrm{H}$ & -2.55109700 & 3.54540800 & -1.33323600 & \\
\hline $\mathrm{H}$ & -3.43958400 & 4.77942600 & -0.41730400 & \\
\hline $\mathrm{H}$ & 4.83391800 & 2.20707000 & -1.23224100 & \\
\hline $\mathrm{H}$ & 5.01579300 & 3.09007900 & 0.29359400 & \\
\hline $\mathrm{H}$ & 3.14578800 & 3.89887500 & -2.01497700 & \\
\hline $\mathrm{H}$ & 3.32694800 & 4.78585800 & -0.48460100 & \\
\hline $\mathrm{H}$ & 4.68774800 & 4.68434400 & -1.61945900 & \\
\hline $\mathrm{N}$ & -0.70319500 & -2.24831900 & -0.83169900 & \\
\hline $\mathrm{C}$ & -0.63908900 & -3.26709900 & 0.01847600 & \\
\hline $\mathrm{C}$ & 0.58558100 & -3.79231800 & 0.43600400 & \\
\hline C & 1.72775300 & -3.66439400 & -0.56761200 & \\
\hline C & 1.68752000 & -2.29946700 & -1.22708700 & \\
\hline C & 2.82731400 & -1.65672500 & -1.70539900 & \\
\hline $\mathrm{C}$ & 2.75302000 & -0.38615400 & -2.27659000 & \\
\hline $\mathrm{C}$ & 1.52192200 & 0.25979200 & -2.37932000 & \\
\hline $\mathrm{C}$ & 0.36667000 & -0.36439600 & -1.91781200 & \\
\hline $\mathrm{C}$ & 0.45898300 & -1.63574600 & -1.35045300 & \\
\hline $\mathrm{H}$ & -1.58875900 & -3.57055300 & 0.45123600 & \\
\hline $\mathrm{H}$ & 0.53840800 & -4.70418500 & 1.02517200 & \\
\hline $\mathrm{H}$ & 1.63550600 & -4.44743800 & -1.33414200 & \\
\hline $\mathrm{H}$ & 3.78821800 & -2.15821000 & -1.61915600 & \\
\hline $\mathrm{H}$ & 3.65595000 & 0.09736900 & -2.63715800 & \\
\hline $\mathrm{H}$ & 1.45791300 & 1.25319600 & -2.81188300 & \\
\hline $\mathrm{H}$ & -0.60210800 & 0.12671400 & -1.97727200 & \\
\hline $\mathrm{H}$ & -1.60331600 & -1.76995400 & -0.97303800 & \\
\hline & -3.65614500 & -0.29410800 & -0.82044000 & \\
\hline \multicolumn{3}{|c|}{ Zero-point correction $=$} & 0.46181 & 9 (Hartree/Particle) \\
\hline \multicolumn{3}{|c|}{ Thermal correction to Energy= } & 0.49 & 0850 \\
\hline \multicolumn{3}{|c|}{ Thermal correction to Enthalpy= } & 0.4 & 91795 \\
\hline \multicolumn{4}{|c|}{ Thermal correction to Gibbs Free Energy= } & 0.400020 \\
\hline \multicolumn{4}{|c|}{ Sum of electronic and zero-point Energies= } & -1559.705314 \\
\hline \multicolumn{4}{|c|}{ Sum of electronic and thermal Energies= } & -1559.676282 \\
\hline \multicolumn{4}{|c|}{ Sum of electronic and thermal Enthalpies= } & -1559.675338 \\
\hline \multicolumn{4}{|c|}{ Sum of electronic and thermal Free Energies $=$} & -1559.767113 \\
\hline \multicolumn{5}{|c|}{ BA-TS4G } \\
\hline $\mathrm{C}$ & -3.39238200 & 0.38364600 & 2.06480200 & \\
\hline $\mathrm{C}$ & 1.06882300 & 4.91031800 & -1.07540500 & \\
\hline $\mathrm{C}$ & 1.25177100 & -1.95281000 & 2.71012800 & \\
\hline
\end{tabular}




\begin{tabular}{|c|c|c|c|}
\hline & & & \\
\hline & -4.22903200 & 60301000 & \\
\hline & & & \\
\hline & -0.60083000 & 2.11587700 & -2.37417000 \\
\hline & -1.43973900 & & \\
\hline & 3.15109500 & & \\
\hline & & & \\
\hline & -1.13254200 & -0.26422100 & \\
\hline & & & \\
\hline & -2.036 & 0.669 & \\
\hline & 0.9 & & \\
\hline & 0.2 & & \\
\hline & & & \\
\hline & & & \\
\hline & 2.8 & & \\
\hline & & & \\
\hline & -2.3 & & -0. \\
\hline & & & \\
\hline & 0.6 & -2.6 & \\
\hline & 2.2 & & \\
\hline & & & \\
\hline & & & \\
\hline & -3.7 & & \\
\hline & & & \\
\hline & & & \\
\hline & & & \\
\hline & -3.8 & & \\
\hline & -4.2 & & \\
\hline & -5.2 & & \\
\hline & & & \\
\hline & 1.5 & & \\
\hline & -0.7 & & \\
\hline & -0.9 & & \\
\hline & -0.2 & & \\
\hline & -0.7 & & \\
\hline & -0.2 & 0.2270 & -1. \\
\hline & -1.1 & & \\
\hline & -2.4 & 1.30348200 & 5900 \\
\hline & -3.0 & -0.04602800 & -1.2 \\
\hline & -4.4 & & -1.2 \\
\hline & 2500 & -1.52893600 & 6800 \\
\hline & -4.05 & & -0.4 \\
\hline & -2.67643100 & -2.33685700 & 09000 \\
\hline & -2.18 & -1.09586900 & \\
\hline & & & -1.72337300 \\
\hline & -1.20082200 & 0.70318800 & -3.25935700 \\
\hline
\end{tabular}




\begin{tabular}{|c|c|c|c|}
\hline & & & \\
\hline & -5.10574100 & & \\
\hline & -6.00050700 & & \\
\hline & & & \\
\hline & -1.97738800 & & \\
\hline & -0.17121300 & & \\
\hline & 2.17912000 & -2.82252700 & \\
\hline & -0.299 & & \\
\hline & & & \\
\hline \multicolumn{3}{|c|}{ Zero-point correction= } & \\
\hline \multicolumn{3}{|c|}{ Thermal correction to Energy= } & \\
\hline \multicolumn{4}{|c|}{ Thermal correction to Enthalpy= } \\
\hline \multicolumn{4}{|c|}{ Thermal correction to Gibbs Free Energy= } \\
\hline \multicolumn{4}{|c|}{ Sum of electronic and zero-point Energies $=$} \\
\hline \multicolumn{4}{|c|}{ Sum of electronic and thermal Energies= } \\
\hline \multirow{2}{*}{\multicolumn{4}{|c|}{$\begin{array}{l}\text { Sum of electronic and thermal Enthalpies }= \\
\text { Sum of electronic and thermal Free Energies= }\end{array}$}} \\
\hline & & & \\
\hline \multicolumn{4}{|c|}{ 3A-TS-GEN } \\
\hline & & & \\
\hline & & & \\
\hline & & & \\
\hline & & & \\
\hline & & & \\
\hline & & & \\
\hline & & & \\
\hline & & & \\
\hline & & & \\
\hline & -0.3 & & \\
\hline & & & \\
\hline & & & \\
\hline & & & \\
\hline & -1.9 & & \\
\hline & & & \\
\hline & -2.0 & & \\
\hline & & & \\
\hline & & & \\
\hline & & & \\
\hline & & & \\
\hline & & & $\pi 0$ \\
\hline & & & \\
\hline & & & \\
\hline & & & \\
\hline & 2.05587900 & -2.79293200 & 4.6323530 \\
\hline & & & \\
\hline & & & \\
\hline
\end{tabular}




\begin{tabular}{|c|c|c|c|}
\hline & -4.77857700 & & \\
\hline & -3.75034500 & & \\
\hline & 3.45544200 & -3.84390600 & \\
\hline & & & \\
\hline & 4.44714000 & -2.82991400 & \\
\hline & -1.01177800 & -5.02244500 & \\
\hline & -0.95615100 & -3.45775700 & \\
\hline & -3.4 & -5.1 & \\
\hline & -3.4 & -3.5 & \\
\hline & -2.62332 & -4.9 & \\
\hline & & & \\
\hline & 3.0 & 0.2 & \\
\hline & & & \\
\hline & 0.7 & 0.1 & -0.4 \\
\hline & 0.8 & -0.0 & \\
\hline & -0.1 & -0.1 & \\
\hline & & & \\
\hline & -0.98 & 1.3 & 0.22 \\
\hline & -1.12 & & \\
\hline & & & \\
\hline & -0.35073 & & \\
\hline & 5.32074 & & \\
\hline & 5.0 & & \\
\hline & & & \\
\hline & & & \\
\hline & 3.2 & & \\
\hline & 4.4 & & \\
\hline & 6.2 & & \\
\hline & 5.7 & & \\
\hline & 3.6 & & \\
\hline & 2.0 & & \\
\hline & 77700 & & \\
\hline & & & \\
\hline & & & \\
\hline & & -1.0 & -0 . \\
\hline & 3.15 & & -0. \\
\hline & & -3.4 & \\
\hline & 5.19806900 & -3.4 & -0.0 \\
\hline & & -2.1 & \\
\hline & & -0.0 & 0000 \\
\hline & 2.11893800 & -2.3 & 3300 \\
\hline & & & \\
\hline & 5.74586800 & -4.3 & 0.16224700 \\
\hline & 2.02620900 & & -2.43173400 \\
\hline & 2.32999800 & 1.48411100 & -3.03586100 \\
\hline & 2.67187400 & 1.45326000 & -4.38683200 \\
\hline
\end{tabular}




\begin{tabular}{|c|c|c|c|c|}
\hline C & 2.72093400 & 2.63370600 & -5.12916100 & \\
\hline $\mathrm{C}$ & 2.42104700 & 3.85036100 & -4.52310400 & \\
\hline $\mathrm{C}$ & 2.07229900 & 3.88692500 & -3.17157500 & \\
\hline $\mathrm{H}$ & 1.75728000 & 2.74140200 & -1.37734100 & \\
\hline $\mathrm{H}$ & 2.90107700 & 0.50128100 & -4.85938400 & \\
\hline $\mathrm{H}$ & 2.99078300 & 2.59943500 & -6.18021300 & \\
\hline $\mathrm{H}$ & 2.45655900 & 4.76949600 & -5.09972200 & \\
\hline $\mathrm{H}$ & 1.83789600 & 4.83369400 & -2.69458600 & \\
\hline $\mathrm{H}$ & 3.72890100 & 1.06815700 & -0.87962300 & \\
\hline $\mathrm{H}$ & 2.58048900 & -0.63832000 & -2.86649800 & \\
\hline $\mathrm{H}$ & 2.65428600 & -0.45295800 & 1.79825200 & \\
\hline $\mathrm{H}$ & 1.30427400 & 0.67457200 & 2.01965300 & \\
\hline 0 & -6.01276900 & 2.46021400 & 1.60834800 & \\
\hline 0 & -5.38288000 & 0.07346200 & 1.35316600 & \\
\hline $\mathrm{F}$ & -5.96804100 & 1.69582400 & -1.17578400 & \\
\hline $\mathrm{S}$ & -4.97837200 & 1.49493200 & 1.24728500 & \\
\hline $\mathrm{C}$ & -4.78650100 & 1.71438100 & -0.56361000 & \\
\hline $\mathrm{F}$ & -4.03756400 & 0.73145400 & -1.07942900 & \\
\hline $\mathrm{F}$ & -4.18573300 & 2.87408000 & -0.83760300 & \\
\hline & -3.62790500 & 1.76591200 & 1.75985300 & \\
\hline \multicolumn{3}{|c|}{ Zero-point correction $=$} & 0.74207 & 9 (Hartree/Particle) \\
\hline \multicolumn{3}{|c|}{ Thermal correction to Energy= } & 0.79 & 3474 \\
\hline \multicolumn{3}{|c|}{ Thermal correction to Enthalpy= } & 0.79 & 94418 \\
\hline \multicolumn{4}{|c|}{ Thermal correction to Gibbs Free Energy= } & 0.650308 \\
\hline \multicolumn{4}{|c|}{ Sum of electronic and zero-point Energies $=$} & -3117.034669 \\
\hline \multicolumn{4}{|c|}{ Sum of electronic and thermal Energies $=$} & -3116.983275 \\
\hline \multicolumn{4}{|c|}{ Sum of electronic and thermal Enthalpies= } & -3116.982330 \\
\hline \multicolumn{4}{|c|}{ Sum of electronic and thermal Free Energies= } & -3117.126440 \\
\hline \multicolumn{5}{|c|}{ C3-Decomposed } \\
\hline $\mathrm{C}$ & -0.81581600 & 1.61002300 & -0.15911000 & \\
\hline $\mathrm{C}$ & -1.21724600 & 0.11774100 & -0.00885200 & \\
\hline $\mathrm{N}$ & -0.19710800 & -0.53450900 & -0.85844800 & \\
\hline $\mathrm{C}$ & 0.35008400 & 0.35656300 & -1.65106500 & \\
\hline $\mathrm{N}$ & -0.05895700 & 1.58892100 & -1.42362200 & \\
\hline $\mathrm{C}$ & 0.64519500 & 2.77162100 & -1.88803300 & \\
\hline $\mathrm{C}$ & 0.01731100 & -1.97958800 & -0.90565200 & \\
\hline $\mathrm{H}$ & 1.08519800 & 0.10093100 & -2.40526000 & \\
\hline $\mathrm{H}$ & 1.21062100 & 2.51803500 & -2.78584200 & \\
\hline $\mathrm{H}$ & 1.33253700 & 3.11444300 & -1.10834800 & \\
\hline $\mathrm{H}$ & -0.07750100 & 3.55558200 & -2.12206200 & \\
\hline $\mathrm{C}$ & -3.02366700 & -4.17799100 & -0.27354600 & \\
\hline $\mathrm{C}$ & -3.70871500 & -4.06172100 & -1.48047600 & \\
\hline $\mathrm{C}$ & -3.18346800 & -3.27403100 & -2.50579100 & \\
\hline $\mathrm{C}$ & -1.97567000 & -2.60785100 & -2.32207100 & \\
\hline & -128254200 & -272259600 & -1.11350200 & \\
\hline
\end{tabular}


Sum of electronic and thermal Enthalpies=

Sum of electronic and thermal Free Energies=

\section{C3-RXT}

$\begin{array}{cccc}\mathrm{C} & -2.67739600 & -1.32500800 & -0.45451300 \\ \mathrm{C} & -2.46632900 & 0.20677700 & -0.37632500 \\ \mathrm{~N} & -1.00612900 & 0.32686500 & -0.58473000 \\ \mathrm{C} & -0.42607600 & -0.85431900 & -0.42218100 \\ \mathrm{~N} & -1.30277300 & -1.83196000 & -0.24992500 \\ \mathrm{C} & -1.02979000 & -3.26094800 & -0.30586500 \\ \mathrm{C} & -0.37713400 & 1.64911300 & -0.55770100 \\ \mathrm{I} & 1.64189700 & -1.16043800 & -0.45507900 \\ \mathrm{H} & -0.11791200 & -3.48984000 & 0.24594000 \\ \mathrm{H} & -0.93146400 & -3.59374400 & -1.34427700 \\ \mathrm{H} & -1.86498000 & -3.77943200 & 0.16675400 \\ \mathrm{C} & -1.72717900 & 4.06726000 & 2.05315200 \\ \mathrm{C} & -1.12211600 & 3.59168300 & 3.21368900 \\ \mathrm{C} & -0.26563200 & 2.49151300 & 3.15192700 \\ \mathrm{C} & -0.01699300 & 1.87045000 & 1.93164400 \\ \mathrm{C} & -0.62074200 & 2.34500300 & 0.76314300 \\ \mathrm{C} & -1.47927500 & 3.44241300 & 0.83102400 \\ \mathrm{H} & -2.39369100 & 4.92316100 & 2.09628500 \\ \mathrm{H} & -1.31400700 & 4.07711800 & 4.16564800 \\ \mathrm{H} & 0.21239600 & 2.12168800 & 4.05370500 \\ \mathrm{H} & 0.65804100 & 1.01740600 & 1.88584700 \\ \mathrm{H} & -1.95285700 & 3.80806700 & -0.07684000 \\ \mathrm{C} & -5.11187600 & 2.50930800 & -1.82055600 \\ \mathrm{C} & -4.32979800 & 1.79608800 & -0.91289400 \\ \mathrm{C} & -3.27956900 & 0.99892800 & -1.36778600 \\ \mathrm{C} & -3.00807700 & 0.92468100 & -2.73740800 \\ \mathrm{C} & -3.78308700 & 1.64433300 & -3.64148000 \\ \mathrm{C} & -4.83821500 & 2.43504800 & -3.18398900 \\ \mathrm{H} & -5.92965700 & 3.12565100 & -1.46045600 \\ \mathrm{H} & -4.53192600 & 1.85801100 & 0.15352700 \\ \mathrm{H} & -2.18387800 & 0.31062400 & -3.09404700 \\ \mathrm{H} & -3.56434500 & 1.58921600 & -4.70329600 \\ \mathrm{H} & -5.44247000 & 2.99478000 & -3.89115500 \\ \mathrm{C} & -3.35380200 & -1.84536200 & 1.91955900 \\ \mathrm{C} & -3.66495400 & -1.85028600 & 0.55624400 \\ \mathrm{C} & -4.91078100 & -2.31076600 & 0.13258100 \\ \mathrm{C} & -5.84559800 & -2.75920700 & 1.06498900 \\ \mathrm{C} & -5.53191200 & -2.75795800 & 2.42157000 \\ \mathrm{C} & -4.28338900 & -2.30242400 & 2.84800700 \\ \mathrm{H} & -2.38156100 & -1.48660300 & 2.25141900 \\ \mathrm{H} & -5.14903500 & -2.31555100 & -0.92793500 \\ \mathrm{H} & -6.81491900 & -3.11387800 & 0.72894700 \\ & & & \end{array}$

$-1960.172152$

$-1960.270387$ 


\begin{tabular}{|c|c|c|c|c|}
\hline $\mathrm{H}$ & -6.25666700 & -3.11309800 & 3.14758600 & \\
\hline $\mathrm{H}$ & -4.03535700 & -2.30289100 & 3.90479700 & \\
\hline $\mathrm{H}$ & -2.68431900 & 0.55719700 & 0.64061700 & \\
\hline $\mathrm{H}$ & -2.99023800 & -1.61202200 & -1.46710600 & \\
\hline $\mathrm{H}$ & -0.80463900 & 2.22921800 & -1.37985700 & \\
\hline $\mathrm{H}$ & 0.68931800 & 1.51954200 & -0.75437500 & \\
\hline $\mathrm{C}$ & 5.17473000 & 0.48830100 & 0.70971900 & \\
\hline $\mathrm{S}$ & 5.10177600 & -0.46773300 & -0.85085100 & \\
\hline $\mathrm{F}$ & 5.87642700 & 1.60841500 & 0.54598900 & \\
\hline $\mathrm{F}$ & 5.74143300 & -0.22785000 & 1.67934600 & \\
\hline $\mathrm{F}$ & 3.94181700 & 0.82400100 & 1.10985500 & \\
\hline 0 & 4.26886000 & -1.63243700 & -0.45346300 & \\
\hline 0 & 4.43582500 & 0.44787600 & -1.78052600 & \\
\hline 0 & 6.49747900 & -0.79199600 & -1.13298000 & \\
\hline \multicolumn{3}{|c|}{ Zero-point correction $=$} & 0.43001 & 0 (Hartree/Particle) \\
\hline \multicolumn{3}{|c|}{ Thermal correction to Energy= } & 0.46 & 61899 \\
\hline \multicolumn{3}{|c|}{ Thermal correction to Enthalpy= } & & 62843 \\
\hline \multicolumn{4}{|c|}{ Thermal correction to Gibbs Free Energy $=$} & 0.357828 \\
\hline \multicolumn{4}{|c|}{ Sum of electronic and zero-point Energies $=$} & -2255.213508 \\
\hline \multicolumn{4}{|c|}{ Sum of electronic and thermal Energies= } & -2255.181618 \\
\hline \multicolumn{4}{|c|}{ Sum of electronic and thermal Enthalpies $=$} & -2255.180674 \\
\hline \multicolumn{4}{|c|}{ Sum of electronic and thermal Free Energies= } & -2255.285690 \\
\hline \multicolumn{5}{|c|}{ C3-XB-INT1BD } \\
\hline $\mathrm{C}$ & -6.67914900 & 3.74444700 & 0.84939900 & \\
\hline $\mathrm{C}$ & -7.14212800 & -2.91630600 & -1.40552700 & \\
\hline $\mathrm{C}$ & -1.99747400 & 2.96913000 & -1.29321100 & \\
\hline $\mathrm{C}$ & -2.24411400 & -1.63537200 & -2.68006800 & \\
\hline $\mathrm{C}$ & -8.12681800 & 3.34907000 & 1.04370200 & \\
\hline $\mathrm{C}$ & -8.53622400 & -2.52819100 & -0.96362700 & \\
\hline 0 & -4.16018200 & 3.90929900 & 0.18930600 & \\
\hline 0 & -4.59986500 & -2.99743100 & -1.99370000 & \\
\hline $\mathrm{C}$ & -5.10936200 & 0.58639400 & -1.17107200 & \\
\hline $\mathrm{C}$ & -4.73489400 & 2.89679000 & -0.18748700 & \\
\hline $\mathrm{C}$ & -5.04037200 & -1.88541200 & -1.73124400 & \\
\hline 0 & -6.05521200 & 2.71489900 & 0.08287400 & \\
\hline 0 & -6.35049300 & -1.72765500 & -1.40542300 & \\
\hline $\mathrm{C}$ & -4.18710100 & 1.75546000 & -0.90945000 & \\
\hline $\mathrm{C}$ & -2.89166700 & 1.75435500 & -1.37269900 & \\
\hline $\mathrm{C}$ & -4.33255100 & -0.61271500 & -1.66955200 & \\
\hline $\mathrm{C}$ & -3.02210900 & -0.49400300 & -2.07067100 & \\
\hline $\mathrm{N}$ & -2.30447300 & 0.66881100 & -1.96838100 & \\
\hline $\mathrm{H}$ & -5.66030300 & 0.32668300 & -0.25789900 & \\
\hline $\mathrm{H}$ & -1.22607400 & 2.89837300 & -2.06364000 & \\
\hline $\mathrm{H}$ & -2.56173500 & 3.89086900 & -1.42540100 & \\
\hline $\mathrm{H}$ & -1.50344000 & 3.03676400 & -0.31539800 & \\
\hline
\end{tabular}




\begin{tabular}{|c|c|c|c|}
\hline & & & \\
\hline & -6.15685600 & 84970000 & \\
\hline & & & \\
\hline & -1.46284100 & -1.22939800 & \\
\hline & & -2.23961400 & \\
\hline & 600 & & \\
\hline & -8.20 & & \\
\hline & -8.64 & 4.11 & \\
\hline & -6.69 & & \\
\hline & -7.14 & -3.35 & \\
\hline & -8.51 & & \\
\hline & -8.96725 & -1.79 & \\
\hline & -9.1 & & \\
\hline & -2.2 & -1.4 & \\
\hline & -3.0 & -2.5 & \\
\hline & -4.4 & -2.4 & \\
\hline & -4.92 & -1.3 & \\
\hline & -4.12 & -0.2 & \\
\hline & -4.6 & & \\
\hline & -3.7 & & \\
\hline & $-2.3 \xi$ & & \\
\hline & $-1.8^{\prime}$ & & \\
\hline & -2.75 & -0.2 & \\
\hline & -2.56 & -3.3 & \\
\hline & -5.0 & & \\
\hline & -6.00 & -1.2 & \\
\hline & -5.66 & & \\
\hline & -4.12 & 2.9 & \\
\hline & -1.7 & & \\
\hline & -0.82 & 0.6 & \\
\hline & -1.2 & -1.5 & \\
\hline & -5.90 & & \\
\hline & 4.48 & -0.5 & \\
\hline & 4.34 & & \\
\hline & 2.8724 & & \\
\hline & 2.31401300 & 79200 & -1.0 \\
\hline & 3.20290400 & -0.3 & \\
\hline & 2.87861800 & -1.28810200 & -2.9 \\
\hline & 2.26394000 & 641700 & \\
\hline & 0.241 & 0.38 & -1.48 \\
\hline & 2.06358200 & -0.870 & 0900 \\
\hline & 2.59348 & -2.27 & -2.5 \\
\hline & 3.76078800 & -1.37985800 & 76700 \\
\hline & 4.02016600 & 4.75548500 & \\
\hline & 3.68442100 & 5.53590900 & 0.85444600 \\
\hline & 2.88213200 & 5.00351600 & -0.15594100 \\
\hline
\end{tabular}




\begin{tabular}{|c|c|c|c|}
\hline $\mathrm{C}$ & 2.41963100 & 3.69445200 & -0.06035500 \\
\hline $\mathrm{C}$ & 2.75251200 & 2.90604900 & 1.04603100 \\
\hline $\mathrm{C}$ & 3.55692800 & 3.44293900 & 2.05124600 \\
\hline $\mathrm{H}$ & 4.64452600 & 5.16499300 & 2.74604100 \\
\hline $\mathrm{H}$ & 4.04486800 & 6.55732000 & 0.77999300 \\
\hline $\mathrm{H}$ & 2.61475500 & 5.60999000 & -1.01583800 \\
\hline $\mathrm{H}$ & 1.79020000 & 3.28258300 & -0.84792900 \\
\hline $\mathrm{H}$ & 3.82140500 & 2.82920300 & 2.90897300 \\
\hline $\mathrm{C}$ & 6.63044200 & 0.45197800 & 3.07175500 \\
\hline $\mathrm{C}$ & 6.03376200 & 0.82190000 & 1.86686900 \\
\hline $\mathrm{C}$ & 4.97065100 & 0.07872200 & 1.35415700 \\
\hline $\mathrm{C}$ & 4.50075700 & -1.03559900 & 2.05566200 \\
\hline $\mathrm{C}$ & 5.09076300 & -1.39836100 & 3.26236100 \\
\hline $\mathrm{C}$ & 6.15791200 & -0.65611500 & 3.77097900 \\
\hline $\mathrm{H}$ & 7.46017700 & 1.03192700 & 3.46389500 \\
\hline $\mathrm{H}$ & 6.39097200 & 1.69240900 & 1.32207000 \\
\hline $\mathrm{H}$ & 3.67416000 & -1.61179100 & 1.64830200 \\
\hline $\mathrm{H}$ & 4.71797200 & -2.26111300 & 3.80599900 \\
\hline $\mathrm{H}$ & 6.61848500 & -0.94177800 & 4.71180300 \\
\hline $\mathrm{C}$ & 5.77068500 & 0.69421100 & -2.85409800 \\
\hline $\mathrm{C}$ & 5.70276300 & -0.36328000 & -1.94221300 \\
\hline $\mathrm{C}$ & 6.79733000 & -1.21441100 & -1.79148100 \\
\hline $\mathrm{C}$ & 7.95516700 & -1.00871400 & -2.54026400 \\
\hline $\mathrm{C}$ & 8.01824600 & 0.04327900 & -3.45074500 \\
\hline $\mathrm{C}$ & 6.92302300 & 0.89347000 & -3.60840500 \\
\hline $\mathrm{H}$ & 4.91623200 & 1.35630000 & -2.97552500 \\
\hline $\mathrm{H}$ & 6.74063800 & -2.03773600 & -1.08392400 \\
\hline $\mathrm{H}$ & 8.80346600 & -1.67458100 & -2.41569500 \\
\hline $\mathrm{H}$ & 8.91695200 & 0.20058000 & -4.03920000 \\
\hline $\mathrm{H}$ & 6.96729800 & 1.71226600 & -4.31998300 \\
\hline $\mathrm{H}$ & 4.76299800 & 1.43730700 & -0.28626200 \\
\hline $\mathrm{H}$ & 4.47896700 & -1.57868600 & -0.65875600 \\
\hline $\mathrm{H}$ & 2.50909500 & 1.04319700 & 2.10835700 \\
\hline $\mathrm{H}$ & 1.18190200 & 1.41694800 & 1.00596100 \\
\hline $\mathrm{C}$ & 1.36627100 & -3.78641600 & 2.08535700 \\
\hline $\mathrm{S}$ & 1.00187300 & -2.56337500 & 0.77186600 \\
\hline $\mathrm{F}$ & 2.40013000 & -3.37951200 & 2.82481100 \\
\hline $\mathrm{F}$ & 1.66583700 & -4.96747000 & 1.55183800 \\
\hline $\mathrm{F}$ & 0.31066400 & -3.93508900 & 2.88348400 \\
\hline 0 & -0.17928000 & -3.12817500 & 0.09413900 \\
\hline 0 & 0.69788700 & -1.33207900 & 1.52489900 \\
\hline 0 & 2.23533300 & -2.53540500 & -0.02126900 \\
\hline \multicolumn{4}{|r|}{$0.884626($ Hartree/Particle $)$} \\
\hline \multicolumn{3}{|c|}{ Thermal correction to Energy= } & 0.945358 \\
\hline \multicolumn{3}{|c|}{ Thermal correction to Enthalpy= } & 0.946303 \\
\hline \multicolumn{4}{|c|}{ Thermal correction to Gibbs Free Energy $=\quad 0.779466$} \\
\hline
\end{tabular}


Sum of electronic and zero-point Energies= Sum of electronic and thermal Energies= Sum of electronic and thermal Enthalpies= Sum of electronic and thermal Free Energies=

\section{C3-XB-INT2G}

C -6.23829100

C -5.94389200

C -1.43712100

C -1.28841100

C -7.68604400

C -7.35597100

$\begin{array}{ll}0 & -3.68468000\end{array}$

$\begin{array}{ll}0 & -3.46916200\end{array}$

C -4.33200700

C -4.17630600

C -4.00715600

$\begin{array}{ll}0 & -5.49146600\end{array}$

$\begin{array}{lll}0 & -5.29773100\end{array}$

C -3.52398900

C -2.23379500

C -3.44386300

C -2.16006000

$\mathrm{N}-1.55647300$

$\mathrm{H}-4.87309100$

$\mathrm{H} \quad-5.14220400$

H -1.06509800

$\mathrm{H}-0.58518700$

$\mathrm{H}-2.04667400$

H $\quad-6.12093800$

$\mathrm{H} \quad-5.83334900$

H -1.84208100

$\mathrm{H} \quad-0.43239700$

$\mathrm{H} \quad-0.92103500$

$\mathrm{H} \quad-8.07491200$

$\mathrm{H}-7.80028700$

$\mathrm{H} \quad-8.28831900$

H $\quad-5.38548500$

$\mathrm{H} \quad-5.93426400$

$\mathrm{H} \quad-7.35367800$

H -7.89421900

$\mathrm{H} \quad-7.89491600$

$\mathrm{N}-3.45172300$

C -3.67472100

C -4.93290800

C -6.09397700
0.94497700 $\begin{array}{ll}-3.59700300 & 2.77866300\end{array}$

$-0.99272100 \quad-3.04918700$

$\begin{array}{ll}-4.22259100 & 0.51567400\end{array}$

$\begin{array}{ll}0.80610900 & -2.24613700\end{array}$

$\begin{array}{ll}-3.05314900 & 2.77774400\end{array}$

$0.64502700-3.10845800$

$\begin{array}{ll}-4.24201100 & 2.23637000\end{array}$

$-2.02575000-0.61659200$

$-0.15781800-2.32820000$

$\begin{array}{ll}-3.50514700 & 1.42247800\end{array}$

$-0.07017200-1.98463700$

$\begin{array}{ll}-3.10334500 & 1.60350800\end{array}$

$-1.26649500-1.64203000$

$-1.63754100-1.94059800$

$\begin{array}{ll}-2.92645200 & 0.21131700\end{array}$

$-3.21422000-0.19475700$

$-2.62617000-1.27340400$

$\begin{array}{ll}-1.32469600 & 0.03292100\end{array}$

$-2.60096000-1.09647000$

$-0.00918000-2.74049100$

$-1.63129200-3.29558400$

$-0.83504500-3.93984800$

$\begin{array}{ll}0.81139700 & -3.74272200\end{array}$

$1.92896900-2.40483900$

$\begin{array}{ll}-5.13334000 & 0.74830800\end{array}$

$-4.46532000-0.11832900$

$\begin{array}{ll}-3.82980600 & 1.47065200\end{array}$

$-0.17885400-2.52107400$

$0.93585500-1.16563800$

$\begin{array}{ll}0.56969300 & -2.74772400\end{array}$

$\begin{array}{ll}-3.27297000 & 3.66332900\end{array}$

$\begin{array}{ll}-4.69141600 & 2.76394300\end{array}$

$\begin{array}{ll}-1.95858200 & 2.79908400\end{array}$

$\begin{array}{ll}-3.38026600 & 1.88351900\end{array}$

$\begin{array}{ll}-3.41346800 & 3.65871300\end{array}$

$\begin{array}{ll}1.19180900 & 0.35690700\end{array}$

$\begin{array}{ll}0.35555700 & 1.30823600\end{array}$

$\begin{array}{ll}0.41384800 & 2.09716000\end{array}$

$0.97752400 \quad 1.27430700$
$-3518.685946$

$-3518.625213$

$-3518.624269$

$-3518.791105$ 


\begin{tabular}{|c|c|c|c|}
\hline & & & \\
\hline & & & \\
\hline & & & \\
\hline & & & \\
\hline & & & \\
\hline & & & \\
\hline & & & \\
\hline & -6.763536 & & \\
\hline & -4.4 & & \\
\hline & -2.8 & 3.23 & \\
\hline & -2.536 & & \\
\hline & -2.884 & -0.3595 & \\
\hline & -4.7 & & \\
\hline & -5.1 & & \\
\hline & -6.9 & & \\
\hline & -6.4 & & \\
\hline & $4.11^{\prime}$ & & \\
\hline & 4.66 & & \\
\hline & 3.50 & & \\
\hline & 2.64 & & \\
\hline & 3.0 & & \\
\hline & & & \\
\hline & 3.560 & & \\
\hline & 0.8299 & -1.7 & \\
\hline & & & \\
\hline & 1.3 & & \\
\hline & 2.77 & & \\
\hline & 6.830 & & \\
\hline & & & \\
\hline & 5.73 & -3.3 & \\
\hline & & -2.5 & \\
\hline & & -1.1 & \\
\hline & 5.775 & & \\
\hline & 7.666 & -0.9 & \\
\hline & 7.63205000 & -3.4 & \\
\hline & 5.71541000 & -4.386 & -2 \\
\hline & 3.84282400 & -2.938 & \\
\hline & 5.790780 & & \\
\hline & 6.97045300 & 3.0957 & \\
\hline & 6.50363600 & & -0. \\
\hline & 5.15347300 & & 200 \\
\hline & & & \\
\hline & 4.73233000 & 3.98183200 & -0.94520200 \\
\hline & & & \\
\hline & & 3.23279400 & \\
\hline & 7.18834700 & 1.00167600 & -0.65635500 \\
\hline
\end{tabular}




\begin{tabular}{|c|c|c|c|c|}
\hline $\mathrm{H}$ & 3.21156200 & 2.58742400 & -0.31172000 & \\
\hline $\mathrm{H}$ & 4.03967600 & 4.81338100 & -1.03214200 & \\
\hline $\mathrm{H}$ & 6.44548000 & 5.13998300 & -1.55056200 & \\
\hline $\mathrm{C}$ & 5.61265300 & -1.44444500 & 2.57029500 & \\
\hline $\mathrm{C}$ & 5.15406500 & -0.12691200 & 2.47824900 & \\
\hline $\mathrm{C}$ & 5.69354800 & 0.85061000 & 3.31405300 & \\
\hline $\mathrm{C}$ & 6.68894400 & 0.51816400 & 4.23183900 & \\
\hline $\mathrm{C}$ & 7.14066900 & -0.79600700 & 4.32410300 & \\
\hline $\mathrm{C}$ & 6.59914900 & -1.77782500 & 3.49340800 & \\
\hline $\mathrm{H}$ & 5.18982000 & -2.20836400 & 1.92150800 & \\
\hline $\mathrm{H}$ & 5.33302000 & 1.87365600 & 3.24268700 & \\
\hline $\mathrm{H}$ & 7.10527500 & 1.28517200 & 4.87752200 & \\
\hline $\mathrm{H}$ & 7.91116100 & -1.05738800 & 5.04288100 & \\
\hline $\mathrm{H}$ & 6.94641000 & -2.80392400 & 3.56525700 & \\
\hline $\mathrm{H}$ & 5.47318200 & -0.43258000 & -0.10217600 & \\
\hline $\mathrm{H}$ & 3.67062800 & 1.22867900 & 1.71427700 & \\
\hline $\mathrm{H}$ & 3.69417800 & 0.76300100 & -2.59039300 & \\
\hline $\mathrm{H}$ & 2.59458500 & -0.62082600 & -2.57044800 & \\
\hline C & -0.48345400 & 3.39419000 & 1.32986400 & \\
\hline $\mathrm{S}$ & -0.11512900 & 1.68908200 & 0.77758200 & \\
\hline $\mathrm{F}$ & 0.08010600 & 3.64102900 & 2.50992500 & \\
\hline $\mathrm{F}$ & -1.80492900 & 3.55004400 & 1.44995500 & \\
\hline $\mathrm{F}$ & -0.03188800 & 4.28294200 & 0.44823900 & \\
\hline 0 & -0.83155200 & 1.61951300 & -0.51782100 & \\
\hline 0 & 1.34781800 & 1.65731300 & 0.68149400 & \\
\hline & -0.70933400 & 0.85565900 & 1.83049100 & \\
\hline \multicolumn{3}{|c|}{ Zero-point correction $=$} & 0.9076 & 28 (Hartree/Particle) \\
\hline \multicolumn{3}{|c|}{ Thermal correction to Energy= } & 0.9 & 68869 \\
\hline \multicolumn{3}{|c|}{ Thermal correction to Enthalpy $=$} & & 69813 \\
\hline \multicolumn{4}{|c|}{ Thermal correction to Gibbs Free Energy= } & 0.801168 \\
\hline \multicolumn{4}{|c|}{ Sum of electronic and zero-point Energies= } & -3519.845380 \\
\hline \multicolumn{4}{|c|}{ Sum of electronic and thermal Energies $=$} & -3519.784139 \\
\hline \multicolumn{4}{|c|}{ Sum of electronic and thermal Enthalpies= } & -3519.783194 \\
\hline \multicolumn{5}{|c|}{ Sum of electronic and thermal Free Energies= } \\
\hline
\end{tabular}

\section{C3-XB-RA-TS1AE}

$\begin{array}{lrrc}\text { C } & -8.23485400 & 1.65728300 & 0.08265900 \\ \mathrm{C} & -5.57812500 & -4.76921100 & -1.00760100 \\ \mathrm{C} & -3.21037000 & 2.69208900 & -1.02375300 \\ \mathrm{C} & -1.40504600 & -1.67507300 & -1.81972000 \\ \mathrm{C} & -9.27153600 & 0.68224600 & 0.59081500 \\ \mathrm{C} & -6.97276500 & -4.95612100 & -0.45610200 \\ 0 & -6.06386600 & 2.79977500 & -0.90217900 \\ 0 & -3.24254200 & -3.79427600 & -1.77124000 \\ \mathrm{C} & -5.04779200 & -0.70248800 & -1.00346200 \\ \mathrm{C} & -6.02639400 & 1.60055000 & -0.72717500\end{array}$




$\begin{array}{cccc}\mathrm{C} & -4.08811500 & -2.99324600 & -1.42692000 \\ 0 & -7.06487100 & 0.89521100 & -0.25930100 \\ \mathrm{O} & -5.31881700 & -3.35621600 & -1.03355500 \\ \mathrm{C} & -4.86223400 & 0.71877500 & -0.98819100 \\ \mathrm{C} & -3.59524200 & 1.24646200 & -1.09310600 \\ \mathrm{C} & -3.92589900 & -1.52618900 & -1.36254800 \\ \mathrm{C} & -2.67797300 & -0.95532700 & -1.49810000 \\ \mathrm{~N} & -2.55891200 & 0.38447400 & -1.31614000 \\ \mathrm{H} & -5.17298100 & -1.03195900 & 0.45674100 \\ \mathrm{H} & -2.16590500 & 2.77762100 & -0.72128200 \\ \mathrm{H} & -3.32668900 & 3.15895600 & -2.00833900 \\ \mathrm{H} & -3.84186300 & 3.22991600 & -0.31878500 \\ \mathrm{H} & -8.57814100 & 2.19508100 & -0.80566400 \\ \mathrm{H} & -7.95783500 & 2.39601300 & 0.84157200 \\ \mathrm{H} & -1.51746300 & -2.25236500 & -2.73926800 \\ \mathrm{H} & -0.58517400 & -0.96143000 & -1.93260100 \\ \mathrm{H} & -1.16604400 & -2.38585500 & -1.02254600 \\ \mathrm{H} & -9.52576300 & -0.04918400 & -0.18148100 \\ \mathrm{H} & -8.90429300 & 0.14733300 & 1.47168600 \\ \mathrm{H} & -10.17996400 & 1.22402400 & 0.86816700 \\ \mathrm{H} & -4.82288400 & -5.25397700 & -0.38194200 \\ \mathrm{H} & -5.48164300 & -5.16397600 & -2.02341500 \\ \mathrm{H} & -7.04457700 & -4.54743900 & 0.55612500 \\ \mathrm{H} & -7.71058300 & -4.45559100 & -1.08948100 \\ \mathrm{H} & -7.21382700 & -6.02201300 & -0.41809600 \\ \mathrm{~N} & -2.02812500 & -0.46288600 & 1.66869500 \\ \mathrm{C} & -2.43115100 & -1.74288800 & 1.56261900 \\ \mathrm{C} & -3.73600000 & -2.17340000 & 1.60312200 \\ \mathrm{C} & -4.80518000 & -1.19395800 & 1.64219800 \\ \mathrm{C} & -4.36420500 & 0.15832400 & 2.04191100 \\ \mathrm{C} & -5.28896600 & 1.15356000 & 2.39350400 \\ \mathrm{C} & -4.88532400 & 2.45388000 & 2.65434200 \\ \mathrm{C} & -3.52316500 & 2.77965100 & 2.55932100 \\ \mathrm{C} & -2.59224600 & 1.81474000 & 2.22095000 \\ \mathrm{C} & -2.98461300 & 0.48119400 & 1.96439600 \\ \mathrm{H} & -1.62792200 & -2.47295700 & 1.44907000 \\ \mathrm{H} & -3.95874000 & -3.23383700 & 1.54873600 \\ \mathrm{H} & -5.75541100 & -1.51637300 & 2.07752900 \\ \mathrm{H} & -6.34223000 & 0.88567800 & 2.44900800 \\ \mathrm{H} & -5.61374200 & 3.21236200 & 2.92318600 \\ \mathrm{H} & -1.53766000 & 2.06949500 & 2.15386800 \\ \mathrm{H} & -6.04498100 & -1.08506600 & -1.19484200 \\ \mathrm{H} & -1426500 & -0.84142100 & -0.05898100\end{array}$




\begin{tabular}{|c|c|c|c|}
\hline & & & \\
\hline & & & \\
\hline & & & \\
\hline & 2.65829900 & & 76000 \\
\hline & 0.41395500 & -0.09820500 & \\
\hline & 6850300 & 2.14479400 & 0700 \\
\hline & & & \\
\hline & 3.67551000 & 2.615 & \\
\hline & & & \\
\hline & 900 & -5.39 & \\
\hline & & & \\
\hline & 3.01 & -3.4 & \\
\hline & & & \\
\hline & & -4.18977 & \\
\hline & & -5.94773 & \\
\hline & & & \\
\hline & $3.3^{\prime}$ & -4.5 & \\
\hline & & -2.7 & \\
\hline & & -4.0 & \\
\hline & & -1.6 & \\
\hline & 627 & -1.50879 & \\
\hline & & -0.71221 & \\
\hline & & $-0 .($ & \\
\hline & 5.20 & -0.2 & \\
\hline & & & \\
\hline & & -2.2 & \\
\hline & & -2.0 & \\
\hline & & & \\
\hline & 4.76 & & \\
\hline & & & \\
\hline & & & \\
\hline & & & \\
\hline & & & \\
\hline & & & \\
\hline & & & \\
\hline & 7.03 & 1.0409 & 7900 \\
\hline & & & \\
\hline & 6.76 & & 2200 \\
\hline & & & \\
\hline & & & \\
\hline & & & 9000 \\
\hline & & -1.1903 & \\
\hline & & & -0.42600300 \\
\hline & & -2.19715900 & \\
\hline & & -2.12735500 & -0.46797300 \\
\hline & 0.51111800 & 3.79887200 & -0.92536900 \\
\hline
\end{tabular}




\begin{tabular}{lrrr}
$\mathrm{S}$ & 1.03039800 & 2.46585500 & -2.06904700 \\
$\mathrm{~F}$ & -0.48075700 & 4.51719200 & -1.45145900 \\
$\mathrm{~F}$ & 1.53122000 & 4.61764200 & -0.66548300 \\
$\mathrm{~F}$ & 0.08279600 & 3.28903800 & 0.23534700 \\
0 & 2.14071500 & 1.83040400 & -1.34443500 \\
0 & -0.18728000 & 1.63846800 & -2.17791500 \\
0 & 1.39786700 & 3.17728500 & -3.29087200 \\
$\mathrm{H}$ & -1.62745600 & 0.79920700 & -1.43812200 \\
Zero-point correction= & \multicolumn{3}{c}{0.880311 (Hartree/Particle) } \\
Thermal correction to Energy= & \multicolumn{3}{c}{0.940519} \\
Thermal correction to Enthalpy= & 0.941463 \\
Thermal correction to Gibbs Free Energy $=$ & 0.774822 \\
Sum of electronic and zero-point Energies $=$ & -3518.668914 \\
Sum of electronic and thermal Energies $=$ & -3518.608705 \\
Sum of electronic and thermal Enthalpies $=$ & -3518.607761 \\
Sum of electronic and thermal Free Energies $=$ & -3518.774403
\end{tabular}

\begin{tabular}{|c|c|c|c|}
\hline \multicolumn{4}{|c|}{ C3-XB-RB-TS2B } \\
\hline & -6.97178500 & 3.47318100 & 0.6753080 \\
\hline & -7.21841700 & & \\
\hline & & & \\
\hline & -2.33647800 & -1.84000800 & -2.67292100 \\
\hline & -8.2 & & \\
\hline & & -2.882 & \\
\hline & -4.60 & & \\
\hline & -4.80 & -3.1 & \\
\hline & -5.14648100 & & \\
\hline & -5.0 & & \\
\hline & -5.19 & -2.11 & \\
\hline & -6.27 & & \\
\hline & -6.43 & -2.0 & \\
\hline & & & \\
\hline & -3.0 & & \\
\hline & -4.4 & & \\
\hline & -3.13 & -0.7 & -2 \\
\hline & & & \\
\hline & -5.2 & -0.0 & \\
\hline & -1.3 & & -1. \\
\hline & -2.7 & & \\
\hline & -2.02 & & \\
\hline & & & \\
\hline & -6.3 & & \\
\hline & -2.9 & -2.4 & \\
\hline & -1.46917100 & -1.43550300 & $-3.1982880 C$ \\
\hline & -1.97976200 & -2.51545900 & \\
\hline & -8.87658500 & 2.50391000 & 0.3851770 \\
\hline
\end{tabular}




\begin{tabular}{|c|c|c|c|}
\hline & -8.07849200 & & \\
\hline & & & \\
\hline & -6.65567300 & -3.99429200 & \\
\hline & -7.39937000 & & \\
\hline & -8.30307700 & & \\
\hline & -9.05 & & \\
\hline & -9.14 & & \\
\hline & -2.23 & -1.0 & \\
\hline & & & \\
\hline & -4.45 & -1.8 & \\
\hline & -4.9 & & \\
\hline & -4.0 & & \\
\hline & -4.3 & & \\
\hline & -3.4 & & \\
\hline & -2.0 & & \\
\hline & -1.6 & & \\
\hline & -2.6 & & \\
\hline & -2.6 & & \\
\hline & -5.1 & -2.7 & \\
\hline & -5.9 & -0.5 & \\
\hline & -5.4 & & \\
\hline & & & \\
\hline & -1.3 & & \\
\hline & -0.6 & & \\
\hline & -1.2 & & \\
\hline & -6.2 & & \\
\hline & $4.4^{\prime}$ & -0.7 & \\
\hline & 4.38 & & \\
\hline & 2.92 & & \\
\hline & 2.33 & & \\
\hline & $3.1^{\prime}$ & -0.5 & \\
\hline & 2.80 & & \\
\hline & 2.35 & 600 & \\
\hline & 0.27 & 0.24 & -1.4 \\
\hline & 2.03 & -1.1 & \\
\hline & 2.44 & -2.48 & -2.2 \\
\hline & 3.68 & -1.79 & \\
\hline & 4.25978400 & & \\
\hline & 3.90054900 & 5.554 & \\
\hline & 3.03 & & -0.6 \\
\hline & 2.53061400 & 2000 & -0.3 \\
\hline & 2.88 & & \\
\hline & 3.75573000 & 3.56903400 & 1.686 \\
\hline & & & \\
\hline & 4.29228000 & 6.55263500 & 0.14893600 \\
\hline & 2.74739400 & 5.52375600 & -1.50096800 \\
\hline
\end{tabular}




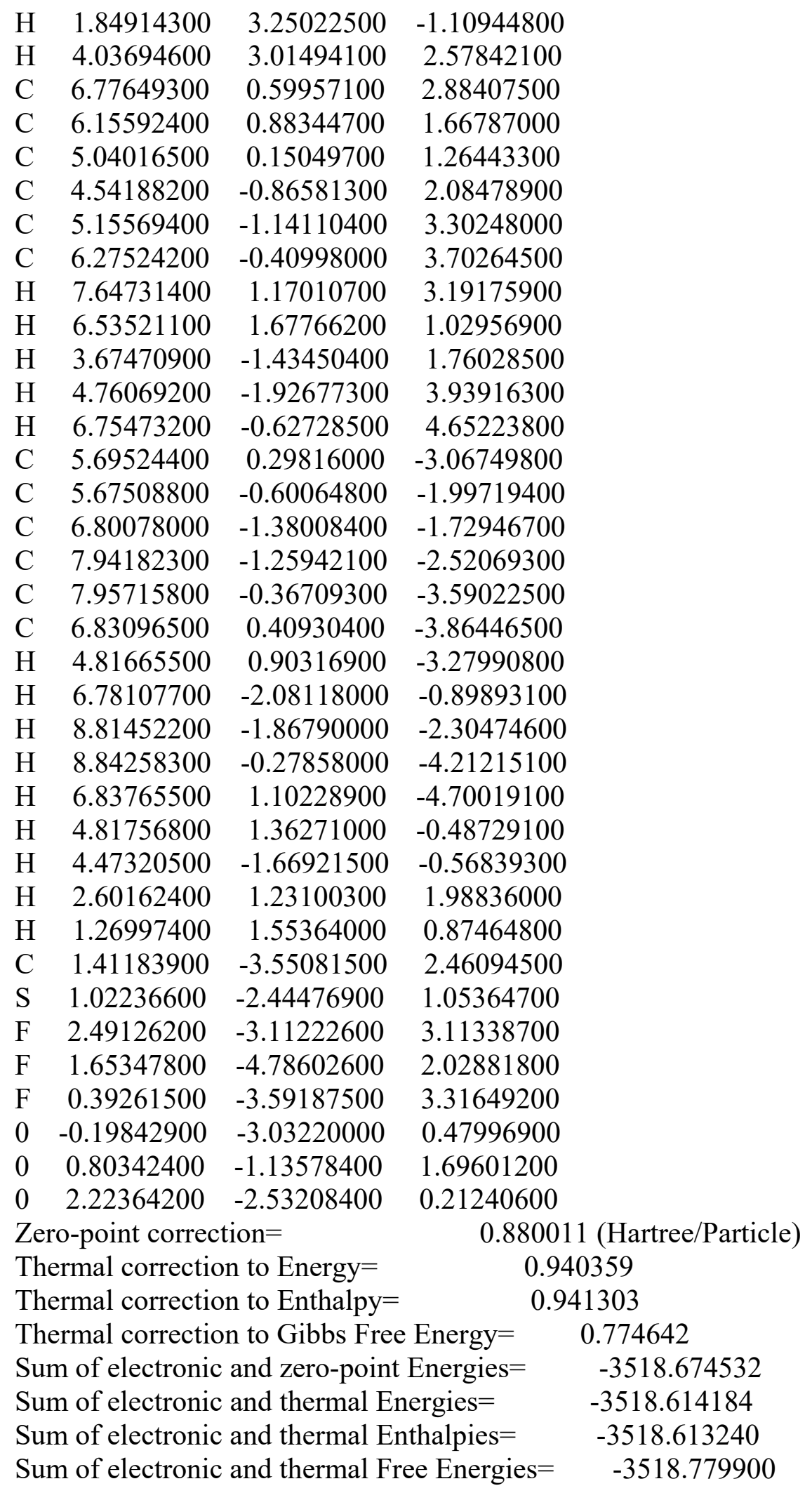

\section{C3-XB-TS1BD}




\begin{tabular}{cccc}
$\mathrm{H}$ & -0.55492000 & 6.18928500 & -1.85475700 \\
$\mathrm{C}$ & -0.23488900 & 5.29578700 & -1.32767100 \\
$\mathrm{C}$ & 0.58320800 & 2.95151300 & 0.01954300 \\
$\mathrm{C}$ & -0.80613900 & 4.04923100 & -1.69255500 \\
$\mathrm{C}$ & 0.70657600 & 5.35344300 & -0.33197500 \\
$\mathrm{C}$ & 1.11999800 & 4.17429200 & 0.33802800 \\
$\mathrm{C}$ & -0.38675400 & 2.88816900 & -1.00046600 \\
$\mathrm{H}$ & 1.14874500 & 6.30485500 & -0.05588500 \\
$\mathrm{H}$ & 1.88179400 & 4.23708200 & 1.10837400 \\
$\mathrm{H}$ & 0.92005700 & 2.03749700 & 0.49749200 \\
$\mathrm{~N}$ & -0.93793500 & 1.68524100 & -1.35505700 \\
$\mathrm{C}$ & -1.83210700 & 1.54732100 & -2.32353800 \\
$\mathrm{H}$ & -2.19993500 & 0.54550600 & -2.53136400 \\
$\mathrm{C}$ & -2.27182800 & 2.66866100 & -3.04021300 \\
$\mathrm{C}$ & -1.76696700 & 3.90756400 & -2.72214700 \\
$\mathrm{H}$ & -2.09384800 & 4.79283200 & -3.26027700 \\
$\mathrm{H}$ & -3.00162700 & 2.53266600 & -3.82815200 \\
$\mathrm{C}$ & -1.91341400 & -0.15348800 & 1.47056400 \\
$\mathrm{C}$ & -3.25229900 & -1.22601700 & -0.71146700 \\
$\mathrm{~N}$ & -1.19396700 & -0.72788700 & 0.44781300 \\
$\mathrm{C}$ & -3.27998700 & -0.22850900 & 1.56101700 \\
$\mathrm{C}$ & -4.08571200 & -0.99432000 & 0.53172000 \\
$\mathrm{C}$ & -1.87995900 & -1.21407400 & -0.63566000 \\
$\mathrm{H}$ & -0.64430000 & 0.85393700 & -0.81251600 \\
$\mathrm{H}$ & -4.45993100 & -1.94638900 & 0.94535400 \\
$\mathrm{H}$ & -4.98847800 & -0.43091200 & 0.27585400 \\
$\mathrm{C}$ & -3.99697000 & 0.36708300 & 2.68417300 \\
$\mathrm{C}$ & -3.95967500 & -1.44565300 & -1.96901500 \\
$\mathrm{C}$ & -1.06570300 & 0.59896600 & 2.46868000 \\
$\mathrm{H}$ & -1.07050200 & 0.10517700 & 3.44637300 \\
$\mathrm{H}$ & -1.44912000 & 1.60842200 & 2.63057600 \\
$\mathrm{H}$ & -0.03741100 & 0.65552500 & 2.10781700 \\
$\mathrm{C}$ & -0.97437500 & -1.72946900 & -1.73121100 \\
$\mathrm{H}$ & -1.51253800 & -1.94782000 & -2.64953600 \\
$\mathrm{H}$ & -0.49819600 & -2.65237000 & -1.37759000 \\
$\mathrm{H}$ & -0.16023700 & -1.02404300 & -1.94131900 \\
0 & -3.53617900 & 0.98895300 & 3.63044400 \\
0 & -3.53303200 & -1.28030500 & -3.10753600 \\
0 & -5.33207400 & 0.13740700 & 2.59243000 \\
0 & -5.24406300 & -1.80948800 & -1.76167100 \\
$\mathrm{C}$ & -6.12835600 & 0.66295700 & 3.65661200 \\
$\mathrm{H}$ & -5.99765900 & 1.74895700 & 3.70387500 \\
$\mathrm{H}$ & -5.78143400 & 0.24581500 & 4.60743400 \\
$\mathrm{H}$ & -1.95873300 & -2.93337000 \\
$\mathrm{H}$ & -2673500 & -2.74231000 & -3.56705100 \\
$\mathrm{H}$ & -1.02390300 & -3.50235900 \\
\hline
\end{tabular}




\begin{tabular}{|c|c|c|c|}
\hline & & & \\
\hline & -7.44611700 & -3.24487700 & \\
\hline & -8.10719600 & -2.43466600 & \\
\hline & -7.85819200 & & \\
\hline & -7.56558800 & & \\
\hline & -7.67717400 & -0.8 & \\
\hline & -7.895 & & \\
\hline & -8.21521 & & \\
\hline & 4.779 & -0.09 & \\
\hline & 3.294 & & \\
\hline & 3.83 & -0.5 & \\
\hline & 3.44 & 3.10 & -2.0 \\
\hline & 3.20 & & \\
\hline & 5.27 & & -1.7 \\
\hline & 4.00 & & \\
\hline & 4.18 & & \\
\hline & 3.08 & 0.67 & -1.1 \\
\hline & & & \\
\hline & 1.73 & 0.6 & -1.7 \\
\hline & 3.99 & & \\
\hline & 1.286 & -1.63 & \\
\hline & & & \\
\hline & 3.84 & -0.5 & \\
\hline & 5.38 & & \\
\hline & & & \\
\hline & 3.33 & -3.6 & \\
\hline & 3.2 & -2.9 & \\
\hline & 2.32 & -4.0 & \\
\hline & 3.9 & -4.5 & \\
\hline & 5.6 & -2.2 & \\
\hline & & -2.6 & \\
\hline & 3.02 & -1.0 & \\
\hline & 2.05 & -0.2 & \\
\hline & 3.22 & -2.3 & \\
\hline & 1.30 & -0.7 & \\
\hline & 1.88 & 0.7 & \\
\hline & 2.46 & -2.8 & \\
\hline & 3.96 & -3.0 & \\
\hline & 1.50 & -2.08 & \\
\hline $\mathrm{H}$ & 0.55 & -0.1 & \\
\hline UI & 2.62 & -3.9 & \\
\hline & 0.91 & -2.47 & \\
\hline & 6.63122700 & -0.97501500 & \\
\hline & & -1.22990600 & \\
\hline & 6.41064700 & 0.21423500 & 0.87990300 \\
\hline & 8.91665700 & -0.30443700 & 1.99357800 \\
\hline
\end{tabular}




\begin{tabular}{|c|c|c|c|c|}
\hline $\mathrm{H}$ & 8.05118500 & -2.15485600 & 2.68612300 & \\
\hline $\mathrm{C}$ & 7.44051600 & 1.14088300 & 0.74522900 & \\
\hline $\mathrm{H}$ & 5.42991700 & 0.41261300 & 0.45550900 & \\
\hline $\mathrm{C}$ & 8.69513900 & 0.88229800 & 1.29833000 & \\
\hline $\mathrm{H}$ & 9.89011500 & -0.50903100 & 2.42856200 & \\
\hline $\mathrm{H}$ & 7.26171300 & 2.06543500 & 0.20470500 & \\
\hline $\mathrm{H}$ & 9.49696000 & 1.60634600 & 1.18980700 & \\
\hline $\mathrm{C}$ & 6.18582800 & -4.13047700 & 0.39284900 & \\
\hline $\mathrm{C}$ & 7.39651300 & -4.18650900 & -0.29840800 & \\
\hline $\mathrm{C}$ & 5.75807800 & -5.24039100 & 1.12729700 & \\
\hline $\mathrm{C}$ & 8.17793300 & -5.34010800 & -0.25269800 & \\
\hline $\mathrm{H}$ & 7.72548400 & -3.32391900 & $-0.8723380 \mathrm{c}$ & \\
\hline $\mathrm{C}$ & 6.53351400 & -6.39554900 & 1.16361800 & \\
\hline $\mathrm{H}$ & 4.81209400 & -5.19920300 & 1.66280600 & \\
\hline $\mathrm{C}$ & 7.74651800 & -6.44602300 & 0.47594600 & \\
\hline $\mathrm{H}$ & 9.11914700 & -5.37541700 & $-0.7924780 \mathrm{c}$ & \\
\hline $\mathrm{H}$ & 6.19174200 & -7.25739600 & 1.72852500 & \\
\hline $\mathrm{H}$ & 8.35082700 & -7.34745600 & 0.50571700 & \\
\hline \multicolumn{3}{|c|}{ Zero-point correction $=$} & 0.8839 & 83 (Hartree/Particle) \\
\hline \multicolumn{3}{|c|}{ Thermal correction to Energy $=$} & & 44435 \\
\hline \multicolumn{3}{|c|}{ Thermal correction to Enthalpy= } & & 45379 \\
\hline \multicolumn{4}{|c|}{ Thermal correction to Gibbs Free Energy $=$} & 0.777817 \\
\hline \multirow{2}{*}{\multicolumn{4}{|c|}{$\begin{array}{l}\text { Sum of electronic and zero-point Energies= } \\
\text { Sum of electronic and thermal Eneroies }=\end{array}$}} & -3518.668989 \\
\hline & \multicolumn{3}{|c|}{ Sum of electronic and thermal Energies= } & -3518.608537 \\
\hline \multicolumn{4}{|c|}{ Sum of electronic and thermal Enthalpies= } & -3518.607593 \\
\hline \multicolumn{4}{|c|}{ Sum of electronic and thermal Free Energies= } & -3518.775155 \\
\hline
\end{tabular}

\section{C3-XB-TS2B}

$\begin{array}{lccc}\mathrm{C} & -6.97178500 & 3.47318100 & 0.67530800 \\ \mathrm{C} & -7.21841700 & -3.19918900 & -1.26946600 \\ \mathrm{C} & -2.25159900 & 2.81394500 & -1.45603000 \\ \mathrm{C} & -2.33647800 & -1.84000800 & -2.67292100 \\ \mathrm{C} & -8.26988100 & 2.90413800 & 1.20253400 \\ \mathrm{C} & -8.50683900 & -2.88274300 & -0.54350600 \\ 0 & -4.60437000 & 3.84648500 & -0.39071600 \\ 0 & -4.80120300 & -3.16715400 & -2.28388000 \\ \mathrm{C} & -5.14648100 & 0.27626300 & -1.05477400 \\ \mathrm{C} & -5.05015800 & 2.71611800 & -0.46126400 \\ \mathrm{C} & -5.19276700 & -2.11452400 & -1.81140100 \\ 0 & -6.27006300 & 2.40399100 & 0.03169800 \\ 0 & -6.43194900 & -2.00276500 & -1.27731800 \\ \mathrm{C} & -4.39696400 & 1.52816500 & -1.03164200 \\ \mathrm{C} & -3.07603700 & 1.55182900 & -1.45045300 \\ \mathrm{C} & -4.46465100 & -0.84287500 & -1.70155700 \\ \mathrm{C} & -3.13214700 & -0.71170500 & -2.07255000 \\ \mathrm{~N} & -2.44576800 & 0.44566600 & -1.92269400\end{array}$




\begin{tabular}{|c|c|c|c|}
\hline & & & \\
\hline & 31708600 & & \\
\hline & -2.79300700 & & \\
\hline & -2.02851700 & & \\
\hline & & & \\
\hline & & & \\
\hline & & & \\
\hline & 17100 & -1.4 & \\
\hline & & & \\
\hline & -8.87 & & \\
\hline & $-8.0^{\prime}$ & & \\
\hline & -8.84 & & \\
\hline & -6.6 & & \\
\hline & -7.3 & -3.5 & \\
\hline & -8.3 & & \\
\hline & -9.0 & & \\
\hline & $-9.1<$ & -3.7 & \\
\hline & -2.2 & & \\
\hline & -3.1 & -2.0 & \\
\hline & -4.4 & -1.8 & \\
\hline & -4.9 & -0.6 & \\
\hline & & & \\
\hline & -4.3 & & \\
\hline & & & \\
\hline & -2.0 & & \\
\hline & -1.6 & & \\
\hline & -2.63 & & \\
\hline & -2.66 & -2.9 & \\
\hline & -5.1 & -2.7 & \\
\hline & -5.9 & -0.5 & \\
\hline & -5.4 & & \\
\hline & & & \\
\hline & -1.3 & & \\
\hline & -0.62 & & \\
\hline & -1.24 & -1.2 & \\
\hline & -6.21 & & -1.27 \\
\hline & 4.47 & -0.7 & \\
\hline & $4.38^{\prime}$ & 0.44 & -0.0603 \\
\hline & 2.92222300 & & \\
\hline & 2.33 & & \\
\hline & 3.17 & -0.5 & -1.7 \\
\hline & 2.80 & -1.5 & -2.7846 \\
\hline & 2.35493300 & 1.58792600 & 0.98474700 \\
\hline & 0.2780 & 0.24376900 & -1.44701900 \\
\hline & 2.03510800 & -1.16639900 & -3.44296100 \\
\hline & 2.44172700 & -2.48045100 & -2.29277300 \\
\hline
\end{tabular}




$\begin{array}{lrrr}\mathrm{H} & 3.68897100 & -1.79866900 & -3.38136700 \\ \mathrm{C} & 4.25978400 & 4.85121400 & 1.46717800 \\ \mathrm{C} & 3.90054900 & 5.55466500 & 0.32024000 \\ \mathrm{C} & 3.03378300 & 4.97625900 & -0.60826000 \\ \mathrm{C} & 2.53061400 & 3.69772000 & -0.38740200 \\ \mathrm{C} & 2.88749300 & 2.98655200 & 0.76266100 \\ \mathrm{C} & 3.75573000 & 3.56903400 & 1.68622100 \\ \mathrm{H} & 4.93343200 & 5.29751100 & 2.19228500 \\ \mathrm{H} & 4.29228000 & 6.55263500 & 0.14893600 \\ \mathrm{H} & 2.74739400 & 5.52375600 & -1.50096800 \\ \mathrm{H} & 1.84914300 & 3.25022500 & -1.10944800 \\ \mathrm{H} & 4.03694600 & 3.01494100 & 2.57842100 \\ \mathrm{C} & 6.77649300 & 0.59957100 & 2.88407500 \\ \mathrm{C} & 6.15592400 & 0.88344700 & 1.66787000 \\ \mathrm{C} & 5.04016500 & 0.15049700 & 1.26443300 \\ \mathrm{C} & 4.54188200 & -0.86581300 & 2.08478900 \\ \mathrm{C} & 5.15569400 & -1.14110400 & 3.30248000 \\ \mathrm{C} & 6.27524200 & -0.40998000 & 3.70264500 \\ \mathrm{H} & 7.64731400 & 1.17010700 & 3.19175900 \\ \mathrm{H} & 6.53521100 & 1.67766200 & 1.02956900 \\ \mathrm{H} & 3.67470900 & -1.43450400 & 1.76028500 \\ \mathrm{H} & 4.76069200 & -1.92677300 & 3.93916300 \\ \mathrm{H} & 6.75473200 & -0.62728500 & 4.65223800 \\ \mathrm{C} & 5.69524400 & 0.29816000 & -3.06749800 \\ \mathrm{C} & 0.80342400 & -1.13578400 & 1.69601200 \\ \mathrm{C} & 5.67508800 & -0.60064800 & -1.99719400 \\ \mathrm{C} & 6.80078000 & -1.38008400 & -1.72946700 \\ \mathrm{C} & 7.94182300 & -1.25942100 & -2.52069300 \\ \mathrm{C} & 7.95715800 & -0.36709300 & -3.59022500 \\ \mathrm{C} & 6.83096500 & 0.40930400 & -3.86446500 \\ \mathrm{H} & 4.81665500 & 0.90316900 & -3.27990800 \\ \mathrm{H} & 6.78107700 & -2.08118000 & -0.89893100 \\ \mathrm{H} & 8.81452200 & -1.86790000 & -2.30474600 \\ \mathrm{H} & 8.84258300 & -0.27858000 & -4.21215100 \\ \mathrm{H} & 6.83765500 & 1.10228900 & -4.70019100 \\ \mathrm{H} & 4.81756800 & 1.36271000 & -0.48729100 \\ \mathrm{H} & 4.47320500 & -1.66921500 & -0.56839300 \\ \mathrm{H} & 1.60162400 & 1.23100300 & 1.98836000 \\ \mathrm{~F} & 1.41183900 & -3.55081500 & 2.46094500 \\ \mathrm{H} & 1.65347800 & -4.78602600 & 2.02881800 \\ \mathrm{H} & -2.53208400 & 0.21240600\end{array}$


Zero-point correction $=$

Thermal correction to Energy=

Thermal correction to Enthalpy=

Thermal correction to Gibbs Free Energy=

Sum of electronic and zero-point Energies=

Sum of electronic and thermal Energies=

Sum of electronic and thermal Enthalpies=

Sum of electronic and thermal Free Energies=

\section{C3-XB-TS3G}

C 7.01036000

C $\quad 4.19587300 \quad-3.99561000$

C $\quad 1.89134000 \quad 3.48072300$

C $\quad 0.00515600 \quad-0.94152500$

C $\quad 8.18944800 \quad 1.62287800$

C $\quad 5.64561400 \quad-4.21606900$

$\mathrm{H} \quad 0.38641300 \quad 1.48820700$

$\begin{array}{lll}0 & 4.63767800 & 3.66952700\end{array}$

$\begin{array}{lll}0 & 1.80551700 & -3.02009500\end{array}$

$\begin{array}{lll}\text { C } & 3.80364500 & 0.05879600\end{array}$

C $\quad 4.65361800 \quad 2.45466500$

C $\quad 2.66932900 \quad-2.20467000$

$\begin{array}{lll}0 & 5.82360300 & 1.77592400\end{array}$

$\begin{array}{lll}0 & 3.94765900 & -2.58940000\end{array}$

C $\quad 3.51129600 \quad 1.54223500$

$\begin{array}{lll}\text { C } & 2.23629800 & 2.01870200\end{array}$

C $\quad 2.53002200 \quad-0.75019500$

C $\quad 1.30052600 \quad-0.17118300$

N $\quad 1.12352900 \quad 1.19816700$

$\mathrm{H} \quad 4.38695800 \quad-0.23852200$

$\mathrm{H} \quad 4.45699900 \quad-0.18046800$

$\mathrm{H} \quad 2.63394400 \quad 4.02029400$

$\mathrm{H} \quad 0.90740900 \quad 3.58379500$

$\mathrm{H} \quad 1.84790000 \quad 3.96337000$

$\mathrm{H} \quad 6.98691700 \quad 3.13006400$

$\mathrm{H} \quad 7.03133700 \quad 3.29304300$

H $\quad 8.15022600 \quad 0.90621600$

H $\quad 8.19418600 \quad 1.06786500$

$\mathrm{H} \quad 9.12297600 \quad 2.18787200$

$\mathrm{H} \quad 3.51878500 \quad-4.51710500$

$\mathrm{H} \quad 3.97804800 \quad-4.34680600$

$\mathrm{H} \quad 5.84123300 \quad-3.85900900$

H $\quad 6.30695700 \quad-3.68284800$

$\mathrm{H} \quad 5.88390200 \quad-5.28257600$

C 1.71748500

C $\quad 3.06001200$
1.01732100

0.99382300

0.62395600

0.96322200

0.95929000

0.62290200

$-0.44977300$

1.09786300

1.42030200

0.92458900

0.97489600

1.14317900

0.91391400

0.91914900

0.89087600

0.76851200

1.00878500

0.91153600

0.75097200

0.03967300

1.77428700

0.03772600

0.15746200

1.60784900

1.95656900

0.19690700

1.78464900

0.01675800

1.03406900

0.30980900

2.00776400

$-0.39274900$

1.31187700

0.66798900

$\begin{array}{ll}0.17832000 & -2.44347000\end{array}$

$-0.18592300-2.50089400$
0.880011 (Hartree/Particle)

0.940359

0.941303

0.774642

$-3518.674532$

$-3518.614184$

$-3518.613240$

$-3518.779900$ 


\begin{tabular}{|c|c|c|c|}
\hline & & & \\
\hline & & & \\
\hline & 1.13406800 & & \\
\hline & 0.76350500 & & \\
\hline & & & \\
\hline & -0.02077800 & & \\
\hline & -0.9666 & & \\
\hline & -0.5992 & 6600 & \\
\hline & & & \\
\hline & 2.7 & -3.5 & -2.2 \\
\hline & & & \\
\hline & & & -2.5 \\
\hline & -1.3 & & \\
\hline & -0.8 & & \\
\hline & -0.2 & & \\
\hline & & & \\
\hline & & & -3.5 \\
\hline & & & \\
\hline & -2.0 & & -1. \\
\hline & -0.4 & & \\
\hline & -5.0 & & \\
\hline & & & \\
\hline & -4.3 & & \\
\hline & -3.3 & & \\
\hline & -3.5 & & \\
\hline & -2.7 & & \\
\hline & -4.4 & & \\
\hline & -1.40 & & 1.7 \\
\hline & & & \\
\hline & -4.4 & & \\
\hline & -1.7 & & \\
\hline & -3.1 & & \\
\hline & -5.4 & & \\
\hline & -3.7 & -0.8 & \\
\hline & -5.5 & -2.7 & \\
\hline & -6.0 & -0.7 & -1.2269 \\
\hline & -6.1 & -1.0 & \\
\hline & -3.8 & 0.6 & -0.069851 \\
\hline & -3.27 & -1.4 & \\
\hline & -3.05 & -1.4 & -1.2530 \\
\hline & -3.5 & & \\
\hline & -5.450 & & \\
\hline & -5.64766100 & 3.65277400 & 3.49407600 \\
\hline & -2.627 & & \\
\hline & -3.32555000 & & \\
\hline & -1.79448500 & 5.44281000 & -1.60158200 \\
\hline
\end{tabular}




\begin{tabular}{|c|c|c|c|c|}
\hline \multirow{2}{*}{$\begin{array}{l}\mathrm{C} \\
\mathrm{H}\end{array}$} & -3.20251200 & 3.26765500 & \multicolumn{2}{|l|}{-2.61106900} \\
\hline & -3.96198900 & 3.00604900 & \multicolumn{2}{|l|}{-0.62489900} \\
\hline $\mathrm{C}$ & -1.66958400 & 5.09568000 & \multicolumn{2}{|l|}{-2.94368400} \\
\hline $\mathrm{H}$ & -1.24382200 & 6.29355800 & \multicolumn{2}{|l|}{-1.20649800} \\
\hline $\mathrm{C}$ & -2.37780800 & 4.00665300 & \multicolumn{2}{|l|}{-3.45440300} \\
\hline $\mathrm{H}$ & -3.75291400 & 2.41240200 & \multicolumn{2}{|l|}{-2.99271900} \\
\hline $\mathrm{H}$ & -1.01935400 & 5.67587900 & \multicolumn{2}{|l|}{-3.59140400} \\
\hline $\mathrm{H}$ & -2.28203800 & 3.73560200 & \multicolumn{2}{|l|}{-4.50131000} \\
\hline $\mathrm{C}$ & -5.40046200 & 5.88910500 & \multicolumn{2}{|l|}{2.19254300} \\
\hline $\mathrm{C}$ & -6.48201200 & 6.60211900 & \multicolumn{2}{|l|}{1.67465800} \\
\hline $\mathrm{C}$ & -4.69808500 & 6.39638100 & \multicolumn{2}{|l|}{3.28964200} \\
\hline $\mathrm{C}$ & -6.86476200 & 7.81165300 & 2.25266800 & \\
\hline $\mathrm{H}$ & -7.02451300 & 6.20696800 & 0.81955800 & \\
\hline $\mathrm{C}$ & -5.07436600 & 7.60928200 & 3.85857400 & \\
\hline $\mathrm{H}$ & -3.85197500 & 5.84450700 & 3.69269500 & \\
\hline $\mathrm{C}$ & -6.16022600 & 8.31726500 & 3.34254300 & \\
\hline $\mathrm{H}$ & -7.70853900 & 8.36019900 & 1.84588700 & \\
\hline $\mathrm{H}$ & -4.52061600 & 8.00250500 & 4.70545100 & \\
\hline $\mathrm{H}$ & -6.45336900 & 9.26258100 & 3.78860300 & \\
\hline $\mathrm{C}$ & -6.82127200 & 2.75308400 & 1.94032600 & \\
\hline $\mathrm{C}$ & -8.02190200 & 3.11285700 & 2.55365300 & \\
\hline $\mathrm{C}$ & -6.83264100 & 1.90271200 & 0.83116700 & \\
\hline $\mathrm{C}$ & -9.23192500 & 2.63114100 & 2.05752700 & \\
\hline $\mathrm{H}$ & -8.00759500 & 3.77127400 & 3.41865800 & \\
\hline $\mathrm{C}$ & -8.04218400 & 1.40821300 & 0.34987200 & \\
\hline $\mathrm{H}$ & -5.89342500 & 1.60776600 & 0.36441000 & \\
\hline $\mathrm{C}$ & -9.24247800 & 1.77623000 & 0.95739300 & \\
\hline $\mathrm{H}$ & -10.16327900 & 2.91604000 & 2.53703400 & \\
\hline $\mathrm{H}$ & -8.04450300 & 0.72872100 & -0.49647000 & \\
\hline $\mathrm{H}$ & -10.18382500 & 1.39109600 & 0.57762700 & \\
\hline \multicolumn{3}{|c|}{ Zero-point correction $=$} & 0.90320 & 0 (Hartree/Particle) \\
\hline \multicolumn{3}{|c|}{ Thermal correction to Energy= } & 0.96 & 63761 \\
\hline \multicolumn{3}{|c|}{ Thermal correction to Enthalpy= } & 0.96 & 64705 \\
\hline \multicolumn{4}{|c|}{ Thermal correction to Gibbs Free Energy= } & 0.798579 \\
\hline \multicolumn{4}{|c|}{ Sum of electronic and zero-point Energies $=$} & -3519.830475 \\
\hline \multicolumn{4}{|c|}{ Sum of electronic and thermal Energies $=$} & -3519.769913 \\
\hline \multicolumn{4}{|c|}{ Sum of electronic and thermal Enthalpies= } & -3519.768969 \\
\hline \multicolumn{4}{|c|}{ Sum of electronic and thermal Free Energies $=$} & -3519.935096 \\
\hline $\mathrm{C}$ & 6.43180300 & 0.99223900 & 2.51084700 & \\
\hline $\mathrm{C}$ & 6.06750300 & -3.88035100 & -2.58002600 & \\
\hline $\mathrm{C}$ & 1.55603000 & -0.78978900 & 3.00287200 & \\
\hline $\mathrm{C}$ & 1.43645200 & -4.36761300 & -0.19329900 & \\
\hline $\mathrm{C}$ & 7.84712300 & 0.85774700 & 1.99547000 & \\
\hline $\mathrm{C}$ & 7.36423400 & -3.13614200 & -2.80961400 & \\
\hline 0 & 3.91566800 & 0.67989500 & 3.12457700 & \\
\hline
\end{tabular}




\begin{tabular}{|c|c|c|c|}
\hline & & & \\
\hline & & & \\
\hline & & & \\
\hline & .16985000 & & \\
\hline & & & \\
\hline & & & \\
\hline & & & \\
\hline & 2.30940700 & & \\
\hline & & & \\
\hline & 6900 & -3.2 & \\
\hline & & & \\
\hline & 4.2 & -0.9 & \\
\hline & & & \\
\hline & 1.2 & & \\
\hline & & & \\
\hline & & -0.7 & \\
\hline & & & \\
\hline & & & \\
\hline & & -5 & \\
\hline & & & \\
\hline & & -4.2 & -1. \\
\hline & & & \\
\hline & & & \\
\hline & & & \\
\hline & & & \\
\hline & & -4.5 & \\
\hline & & -2. & -3 . \\
\hline & & -3 & \\
\hline & & -3. & \\
\hline & & & \\
\hline & & & \\
\hline & & & \\
\hline & & & \\
\hline & & & \\
\hline & & & \\
\hline & & 4.316 & 7900 \\
\hline & & & \\
\hline & 244600 & 3.244 & \\
\hline & 4.32542300 & 2.25211100 & \\
\hline & & & -0.5 \\
\hline & & & 57000 \\
\hline & & & \\
\hline & & 3.20076100 & 1.25623800 \\
\hline & & & \\
\hline & & -0.26050100 & -1.64767700 \\
\hline & 4.59105200 & 1.27883300 & -2.95374400 \\
\hline
\end{tabular}




\begin{tabular}{lccc}
$\mathrm{H}$ & 5.16422100 & -0.37585700 & -2.69259500 \\
$\mathrm{H}$ & 6.88365400 & 1.42648100 & -2.04056000 \\
$\mathrm{H}$ & 6.47144900 & 0.30928000 & -0.73371700 \\
$\mathrm{C}$ & -4.16836600 & 0.22831000 & -1.47031300 \\
$\mathrm{C}$ & -4.71873400 & 0.27155600 & -0.02666000 \\
$\mathrm{~N}$ & -3.54737400 & -0.19637600 & 0.74635600 \\
$\mathrm{C}$ & -2.68212600 & -0.79239800 & -0.05989500 \\
$\mathrm{~N}$ & -3.04546500 & -0.72072000 & -1.33356800 \\
$\mathrm{C}$ & -2.17453700 & -0.97957800 & -2.47185000 \\
$\mathrm{C}$ & -3.60738400 & -0.25917200 & 2.20381000 \\
$\mathrm{I}$ & -0.86495800 & -1.65156700 & 0.56724900 \\
$\mathrm{H}$ & -1.69322100 & -1.95187800 & -2.35674600 \\
$\mathrm{H}$ & -1.41556000 & -0.19507600 & -2.55703200 \\
$\mathrm{H}$ & -2.79674500 & -1.00267900 & -3.36772400 \\
$\mathrm{C}$ & -6.85863700 & -1.51697600 & 3.73800800 \\
$\mathrm{C}$ & -6.77361800 & -2.88429400 & 3.48872200 \\
$\mathrm{C}$ & -5.65853500 & -3.40332900 & 2.82898800 \\
$\mathrm{C}$ & -4.63353200 & -2.55488200 & 2.42186900 \\
$\mathrm{C}$ & -4.71236100 & -1.18136800 & 2.67182400 \\
$\mathrm{C}$ & -5.83122000 & -0.66740500 & 3.32719300 \\
$\mathrm{H}$ & -7.72456100 & -1.10840900 & 4.24985100 \\
$\mathrm{H}$ & -7.57286500 & -3.54641900 & 3.80735500 \\
$\mathrm{H}$ & -7.17155200 & 1.19566000 & -4.89507500 \\
$\mathrm{H}$ & -5.58701400 & -4.46931000 & 2.63593200 \\
$\mathrm{H}$ & -3.76237800 & -2.96215100 & 1.91130900 \\
$\mathrm{H}$ & -5.89674200 & 0.40136800 & 3.51609600 \\
$\mathrm{C}$ & -7.06162200 & 3.04120000 & 1.08752000 \\
$\mathrm{C}$ & -6.57805800 & 1.79132000 & 0.70205200 \\
$\mathrm{C}$ & -5.22483200 & 1.62266400 & 0.40938600 \\
$\mathrm{C}$ & -4.35028900 & 2.71010000 & 0.50984900 \\
$\mathrm{C}$ & -4.83424300 & 3.95426500 & 0.90325100 \\
$\mathrm{C}$ & -6.18977800 & 4.12223900 & 1.19038000 \\
$\mathrm{H}$ & -8.11660700 & 3.16671700 & 1.31070500 \\
$\mathrm{H}$ & -7.25196500 & 0.94127200 & 0.62925100 \\
$\mathrm{H}$ & -3.29413800 & 2.57502100 & 0.28300800 \\
$\mathrm{H}$ & -4.15251700 & 4.79523400 & 0.98548100 \\
$\mathrm{C}$ & -6.56351000 & 5.09483100 & 1.49573600 \\
$\mathrm{C}$ & -5.19678100 & -0.17878300 & -2.49349500 \\
$\mathrm{H}$ & -5.75256000 & 0.78815500 & -3.33095700 \\
$\mathrm{H}$ & -1.16051900 & -5.05576100 \\
\hline
\end{tabular}




\begin{tabular}{lrrrr}
$\mathrm{H}$ & -6.94349000 & -2.88767400 & -3.57510800 \\
$\mathrm{H}$ & -5.51532900 & -0.47593100 & 0.08949600 \\
$\mathrm{H}$ & -3.73493500 & 1.20280500 & -1.73387900 \\
$\mathrm{H}$ & -3.77609300 & 0.75673400 & 2.57137900 \\
$\mathrm{H}$ & -2.63061000 & -0.58867800 & 2.56490200 \\
$\mathrm{C}$ & 0.40865100 & 3.42095300 & -1.30118700 \\
$\mathrm{~S}$ & 0.05692600 & 1.70803900 & -0.76167000 \\
$\mathrm{~F}$ & -0.13554100 & 3.66050300 & -2.49291100 \\
$\mathrm{~F}$ & 1.72699700 & 3.60668200 & -1.39222500 \\
$\mathrm{~F}$ & -0.08244700 & 4.29780900 & -0.42755300 \\
0 & 0.75413500 & 1.63527200 & 0.54106700 \\
0 & -1.40921900 & 1.66216700 & -0.68675500 \\
0 & 0.66479200 & 0.88464300 & -1.81502400 \\
Zero-point correction= & \multicolumn{4}{c}{0.904963 (Hartree/Particle) } \\
Thermal correction to Energy= & \multicolumn{2}{c}{0.965546} \\
Thermal correction to Enthalpy= & 0.966490 \\
Thermal correction to Gibbs Free Energy= & 0.799249 \\
Sum of electronic and zero-point Energies $=$ & -3519.842699 \\
Sum of electronic and thermal Energies $=$ & -3519.782116 \\
Sum of electronic and thermal Enthalpies $=$ & -3519.781172 \\
Sum of electronic and thermal Free Energies $=$ & -3519.948413
\end{tabular}

$\begin{array}{lrcc}\text { C4-BA-TS-GEN } & & \\ \mathrm{C} & 3.64776300 & -3.74175100 & -0.61218500 \\ \mathrm{C} & 3.61653300 & 3.26467600 & -1.36905000 \\ \mathrm{C} & -1.38658700 & -2.75828400 & -1.76047600 \\ \mathrm{C} & -1.37483200 & 1.98585400 & -2.49171000 \\ \mathrm{C} & 5.09072900 & -3.29954900 & -0.53417600 \\ \mathrm{C} & 5.01678600 & 2.87984500 & -0.95211400 \\ \mathrm{H} & -2.15337100 & -0.38557800 & -2.09403500 \\ 0 & 1.03388000 & -3.88056800 & -0.94923400 \\ 0 & 1.08863200 & 3.27220900 & -2.13477000 \\ \mathrm{C} & 1.51369100 & -0.25570300 & -1.21802300 \\ \mathrm{C} & 1.53906900 & -2.78249200 & -1.05903000 \\ \mathrm{C} & 1.55795000 & 2.20292200 & -1.79704800 \\ 0 & 2.85979400 & -2.57575100 & -0.91536700 \\ 0 & 2.83297300 & 2.06050000 & -1.40100300 \\ \mathrm{C} & 0.82262700 & -1.52343800 & -1.35115900 \\ \mathrm{C} & -0.51788800 & -1.54331700 & -1.67189900 \\ \mathrm{C} & 0.83179700 & 0.91858700 & -1.74037400 \\ \mathrm{C} & -0.50883900 & 0.84897500 & -2.04073700 \\ \mathrm{~N} & -1.13238000 & -0.36300000 & -1.96101600 \\ \mathrm{H} & 1.52498100 & 0.00282900 & 0.10509100 \\ \mathrm{H} & 2.59542100 & -0.27628800 & -1.33095900 \\ \mathrm{H} & -1.46994200 & -3.22992200 & -0.77861300 \\ \mathrm{H} & -2.38542500 & -2.48251400 & -2.10182100\end{array}$




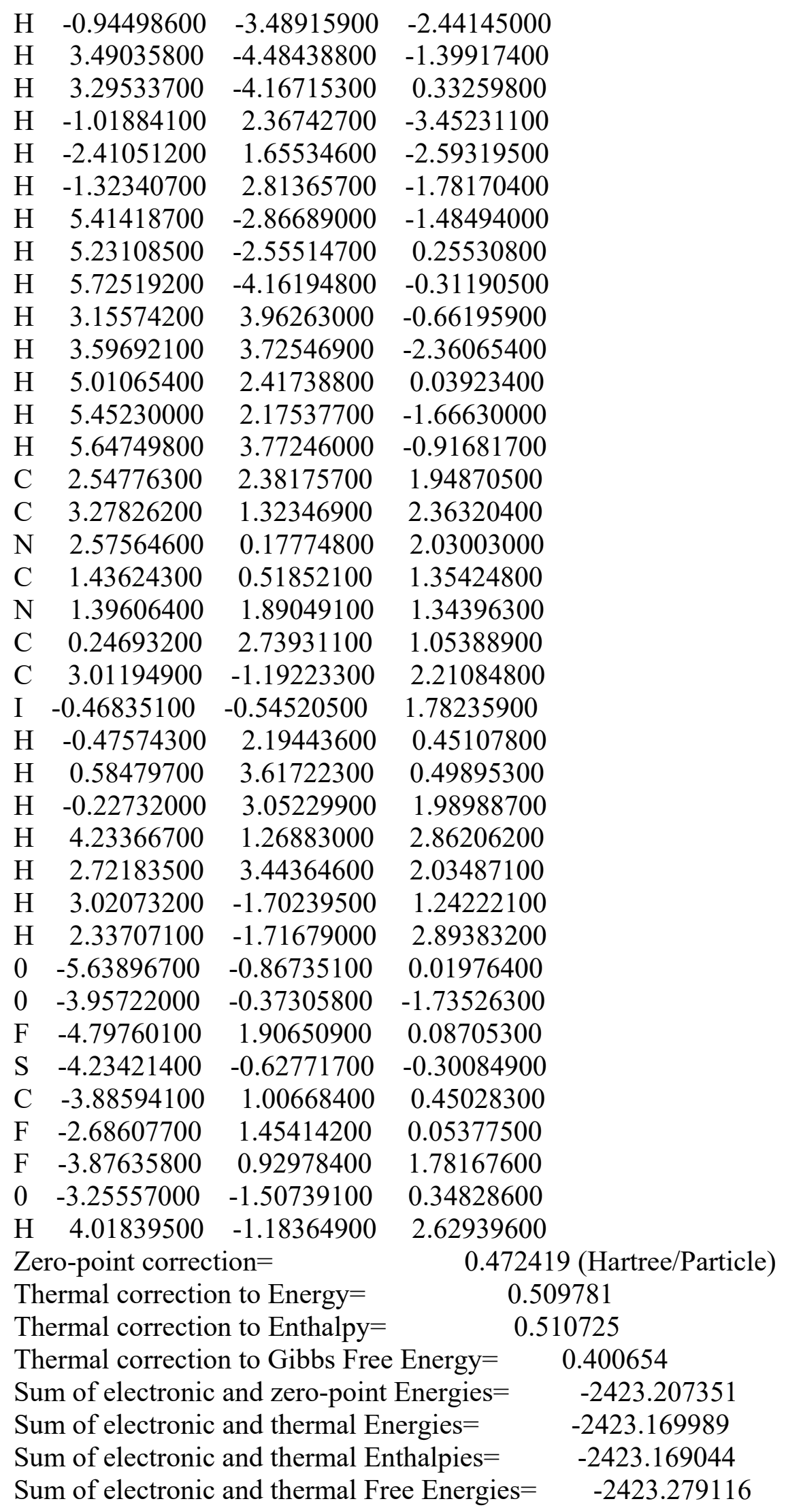




\begin{tabular}{lccc}
\multicolumn{4}{l}{ C4-RXT } \\
C & -2.64798000 & 0.11512700 & 0.01475700 \\
N & -3.72304100 & -0.68541200 & 0.01029600 \\
C & -4.85539600 & 0.07881700 & 0.18280700 \\
C & -4.45026400 & 1.37089900 & 0.29391000 \\
N & -3.07764100 & 1.37328200 & 0.18637400 \\
C & -3.70056700 & -2.13780100 & -0.15061200 \\
H & -3.27480600 & -2.39385100 & -1.12230700 \\
H & -4.72810400 & -2.49349300 & -0.09271200 \\
H & -3.10979600 & -2.58721100 & 0.64930300 \\
C & -2.22532200 & 2.55860900 & 0.25514900 \\
H & -2.87243400 & 3.42664500 & 0.37111500 \\
H & -1.64894100 & 2.65069000 & -0.66698600 \\
H & -1.55363900 & 2.47981500 & 1.11173300 \\
I & -0.66947500 & -0.47633700 & -0.19880600 \\
H & -5.83788100 & -0.36577400 & 0.20944700 \\
H & -5.00697600 & 2.28352300 & 0.43828100 \\
0 & 4.13631200 & -1.40887400 & 0.37740200 \\
F & 3.68755800 & 0.85578900 & -1.48527900 \\
F & 4.03975800 & 1.57568800 & 0.52269900 \\
S & 2.83431400 & -0.74725700 & 0.40372300 \\
C & 3.17672700 & 0.92608100 & -0.25702000 \\
0 & 1.85870700 & -1.27029400 & -0.58637200 \\
0 & 2.25609400 & -0.48086200 & 1.72306200 \\
F & 2.04898000 & 1.64513200 & -0.31822400 \\
\hline
\end{tabular}

$\begin{array}{lc}\text { Zero-point correction }= & 0.161521 \text { (Hartree/Particle) } \\ \text { Thermal correction to Energy }= & 0.178999 \\ \text { Thermal correction to Enthalpy= } & 0.179944 \\ \text { Thermal correction to Gibbs Free Energy= } & 0.111455 \\ \text { Sum of electronic and zero-point Energies }= & -1561.400328 \\ \text { Sum of electronic and thermal Energies }= & -1561.382849 \\ \text { Sum of electronic and thermal Enthalpies }= & -1561.381905 \\ \text { Sum of electronic and thermal Free Energies }= & -1561.450394\end{array}$

\begin{tabular}{lrrr}
\multicolumn{4}{c}{ C4-XB-INT1BD } \\
H & -3.71588800 & 0.16254600 & 3.01590700 \\
$\mathrm{C}$ & -2.68643100 & 0.02237000 & 2.69918700 \\
$\mathrm{C}$ & -0.00943300 & -0.31239100 & 1.85954500 \\
$\mathrm{C}$ & -2.13736900 & 0.92485200 & 1.75273000 \\
$\mathrm{C}$ & -1.92094000 & -1.00256100 & 3.19580800 \\
$\mathrm{C}$ & -0.57692200 & -1.16368400 & 2.77757900 \\
$\mathrm{C}$ & -0.80202800 & 0.72703200 & 1.32874500 \\
$\mathrm{H}$ & -2.34002600 & -1.69859500 & 3.91428000 \\
$\mathrm{H}$ & 0.01654100 & -1.97073800 & 3.19552800 \\
$\mathrm{H}$ & 1.03041200 & -0.40655000 & 1.55477700
\end{tabular}




\begin{tabular}{|c|c|c|c|}
\hline & 7833900 & & \\
\hline & & & \\
\hline & & & \\
\hline & & & \\
\hline & -2.86200900 & & \\
\hline & -3.88 & & \\
\hline & -2.81472300 & & \\
\hline & -1.66099700 & -2.1 & \\
\hline & -2.67 & & \\
\hline & -0.96 & -1.4 & \\
\hline & -2.8 & & \\
\hline & -3.5 & -0.5 & \\
\hline & -1.4 & & \\
\hline & 0.7 & & \\
\hline & -4.4 & -0.8 & \\
\hline & -3.9 & & \\
\hline & -3.52 & -2.4 & \\
\hline & -3.1 & & \\
\hline & -0.9 & -3.4 & \\
\hline & -1.7 & & \\
\hline & -0.4 & -3.2 & \\
\hline & -0.2 & -3.7 & \\
\hline & -0.5 & & \\
\hline & -1.1 & & \\
\hline & & & \\
\hline & 0.0 & & \\
\hline & -3.10 & $-3.3 c$ & \\
\hline & -2.52 & & \\
\hline & -4.73 & -1.8 & \\
\hline & -4.41 & & \\
\hline & -5.4 & -2.4 & \\
\hline & -4.8 & & \\
\hline & -5.5 & -3.5 & \\
\hline & -4.97 & & -2 \\
\hline & -5.02 & & \\
\hline & -4.32 & 3.84 & 7600 \\
\hline & -6.35 & & \\
\hline & -6.98 & & \\
\hline & -6.82655200 & 4.06864200 & \\
\hline & $-6.2 \varepsilon$ & 3.08 & -0 \\
\hline & -6.73 & 333000 & 3500 \\
\hline & -7.33 & & \\
\hline & -6.54801400 & -0.61992100 & 38700 \\
\hline & -7.30 & -2.08404900 & \\
\hline & & -3.62174000 & 1.43669900 \\
\hline & 3.03102800 & -2.55180500 & 1.55133000 \\
\hline
\end{tabular}




\begin{tabular}{|c|c|c|c|c|}
\hline $\mathrm{C}$ & 3.68530100 & -3.10140200 & 0.87009500 & \\
\hline $\mathrm{H}$ & 3.10578300 & -3.82362000 & 0.29298200 & \\
\hline $\mathrm{F}$ & 1.68188400 & 2.39597700 & 2.49183600 & \\
\hline 0 & 3.33048000 & 0.19428200 & 1.44799700 & \\
\hline $\mathrm{F}$ & 3.73009700 & 2.54707600 & 3.17643400 & \\
\hline $\mathrm{C}$ & 2.93699600 & 2.66537500 & 2.11532400 & \\
\hline $\mathrm{N}$ & 4.34123900 & -2.15979800 & -0.02823000 & \\
\hline $\mathrm{H}$ & 6.34307200 & -2.23251300 & 0.75172800 & \\
\hline $\mathrm{S}$ & 3.43986400 & 1.50426600 & 0.79399800 & \\
\hline $\mathrm{C}$ & 5.66089200 & -1.77526700 & 0.05234900 & \\
\hline 0 & 2.40328400 & 1.75200800 & -0.24162600 & \\
\hline $\mathrm{F}$ & 2.98372100 & 3.92439700 & 1.68659500 & \\
\hline I & 1.65852500 & -1.48546500 & -1.39873200 & \\
\hline $\mathrm{C}$ & 3.72002900 & -1.42627100 & -0.96142600 & \\
\hline 0 & 4.78120500 & 1.93309700 & 0.40556500 & \\
\hline $\mathrm{C}$ & 5.84262700 & -0.79120000 & -0.86489800 & \\
\hline $\mathrm{N}$ & 4.62872400 & -0.59037200 & -1.48419700 & \\
\hline $\mathrm{H}$ & 6.71404800 & -0.21375900 & -1.13006400 & \\
\hline $\mathrm{C}$ & 4.37010300 & 0.39658500 & -2.52920400 & \\
\hline $\mathrm{H}$ & 3.54864000 & 1.04394500 & -2.21721800 & \\
\hline $\mathrm{H}$ & 4.12470900 & -0.11116700 & -3.46422400 & \\
\hline $\mathrm{H}$ & 5.27487300 & 0.98988000 & -2.65644200 & \\
\hline \multicolumn{3}{|c|}{ Zero-point correction $=$} & 0.61536 & 88 (Hartree/Particle) \\
\hline \multicolumn{3}{|c|}{ Thermal correction to Energy= } & 0.66 & 1844 \\
\hline \multicolumn{3}{|c|}{ Thermal correction to Enthalpy= } & 0.66 & 62788 \\
\hline \multicolumn{4}{|c|}{ Thermal correction to Gibbs Free Energy $=$} & 0.529834 \\
\hline \multicolumn{4}{|c|}{ Sum of electronic and zero-point Energies $=$} & -2824.867591 \\
\hline \multicolumn{4}{|c|}{ Sum of electronic and thermal Energies $=$} & -2824.821115 \\
\hline \multicolumn{4}{|c|}{ Sum of electronic and thermal Enthalpies= } & -2824.820171 \\
\hline \multicolumn{4}{|c|}{ Sum of electronic and thermal Free Energies= } & -2824.953125 \\
\hline
\end{tabular}

$\begin{array}{lccc}\text { C4-XB-INT2G } & & \\ \mathrm{C} & 4.38383000 & 2.38324100 & 1.77163500 \\ \mathrm{C} & 5.38152100 & -3.75405000 & -1.80550900 \\ \mathrm{C} & 0.09498900 & -0.33483300 & 2.84785700 \\ \mathrm{C} & 0.70097900 & -4.40136500 & 0.37541200 \\ \mathrm{C} & 5.72499900 & 2.53554800 & 1.08917000 \\ \mathrm{C} & 6.69170000 & -3.00873300 & -1.93516100 \\ 0 & 1.94401600 & 1.66071100 & 2.38211400 \\ 0 & 2.96389400 & -4.55767700 & -1.21628900 \\ \mathrm{C} & 3.24333100 & -1.43615300 & 0.78113100 \\ \mathrm{C} & 2.63595200 & 0.79016100 & 1.86796200 \\ \mathrm{C} & 3.32957600 & -3.53425300 & -0.65688100 \\ 0 & 3.92413000 & 1.05184200 & 1.51611400 \\ 0 & 4.56717600 & -3.02432600 & -0.88464800 \\ \mathrm{C} & 2.24470700 & -0.56316200 & 1.50980300\end{array}$




\begin{tabular}{|c|c|c|c|}
\hline & & & \\
\hline & & & \\
\hline & 1.34638200 & -3.08647200 & \\
\hline & & & \\
\hline & 3.69236900 & -0.89461400 & \\
\hline & & -1.67660700 & \\
\hline & -0.46495200 & & \\
\hline & & & \\
\hline & & & \\
\hline & & 2.5 & \\
\hline & & & \\
\hline & 1.4 & -5.2 & \\
\hline & & & \\
\hline & & -4.3 & \\
\hline & & & \\
\hline & 5.62 & 2.3 & \\
\hline & & & \\
\hline & & -3.8 & \\
\hline & & -4.7 & \\
\hline & & & \\
\hline & & -2.9 & \\
\hline & & -3.5 & \\
\hline & & & \\
\hline & & & \\
\hline & & & \\
\hline & & & \\
\hline & & & \\
\hline & 3.47 & & \\
\hline & & & \\
\hline & & & \\
\hline & 1.1 & 4.2 & \\
\hline & & & \\
\hline & 4.36 & & \\
\hline & & & \\
\hline & & & \\
\hline & & & \\
\hline & & & \\
\hline & & 0.00 & \\
\hline & & & \\
\hline & & -0.593 & -1.71424700 \\
\hline & & & \\
\hline & & & \\
\hline & -5.40746200 & -1.404 & \\
\hline & & -1.33197000 & -0.50171700 \\
\hline & -4.98022800 & -1.57304100 & 0.41543100 \\
\hline & -3.82465100 & -1.77403300 & -0.23027800 \\
\hline
\end{tabular}




\begin{tabular}{|c|c|c|c|c|}
\hline N & -4.07219300 & -1.68127300 & -1.54345600 & \\
\hline $\mathrm{C}$ & -3.08163600 & -1.84094400 & -2.60329900 & \\
\hline $\mathrm{C}$ & -5.12320300 & -1.46163300 & 1.86210700 & \\
\hline I & -1.94499000 & -2.08612700 & 0.64981600 & \\
\hline $\mathrm{H}$ & -2.67163400 & -2.85239400 & -2.57726100 & \\
\hline $\mathrm{H}$ & -2.29344300 & -1.10028300 & -2.46396400 & \\
\hline $\mathrm{H}$ & -3.58322700 & -1.67397400 & -3.55589400 & \\
\hline $\mathrm{H}$ & -6.99087100 & -1.13164600 & -0.19563900 & \\
\hline $\mathrm{H}$ & -5.82611800 & -1.29175200 & -2.72130200 & \\
\hline $\mathrm{H}$ & -6.18600600 & -1.39606200 & 2.09197700 & \\
\hline $\mathrm{H}$ & -4.61282500 & -0.55695000 & 2.19964800 & \\
\hline $\mathrm{C}$ & -3.04165000 & 3.31251400 & -0.73464200 & \\
\hline $\mathrm{S}$ & -2.82751100 & 1.60718500 & -0.10744500 & \\
\hline $\mathrm{F}$ & -3.82003800 & 3.32513300 & -1.81492900 & \\
\hline $\mathrm{F}$ & -1.85116100 & 3.82142400 & -1.06549200 & \\
\hline $\mathrm{F}$ & -3.59011000 & 4.09416900 & 0.19236000 & \\
\hline 0 & -1.88381100 & 1.80909100 & 1.01725200 & \\
\hline 0 & -4.17220400 & 1.18968900 & 0.29028600 & \\
\hline 0 & -2.24558200 & 0.90426300 & -1.25938600 & \\
\hline $\mathrm{H}$ & -4.69849200 & -2.34294600 & 2.34492300 & \\
\hline \multicolumn{3}{|c|}{ Zero-point correction $=$} & 0.63893 & 30 (Hartree/Particle) \\
\hline \multicolumn{3}{|c|}{ Thermal correction to Energy= } & 0.68 & 35787 \\
\hline \multicolumn{3}{|c|}{ Thermal correction to Enthalpy= } & 0.68 & 86731 \\
\hline \multicolumn{4}{|c|}{ Thermal correction to Gibbs Free Energy $=$} & 0.553292 \\
\hline \multicolumn{4}{|c|}{ Sum of electronic and zero-point Energies $=$} & -2826.031603 \\
\hline \multicolumn{4}{|c|}{ Sum of electronic and thermal Energies $=$} & -2825.984746 \\
\hline \multicolumn{4}{|c|}{ Sum of electronic and thermal Enthalpies $=$} & -2825.983802 \\
\hline \multicolumn{4}{|c|}{ Sum of electronic and thermal Free Energies= } & -2826.117241 \\
\hline \multicolumn{5}{|c|}{ C4-XB-RA-TS1AE } \\
\hline $\mathrm{C}$ & -5.99459900 & 2.80492700 & -0.02677100 & \\
\hline $\mathrm{C}$ & -4.91504700 & -4.07792000 & -1.00379500 & \\
\hline $\mathrm{C}$ & -0.86397100 & 2.61812800 & -1.08913200 & \\
\hline $\mathrm{C}$ & -0.12324500 & -2.06280600 & -1.80540200 & \\
\hline $\mathrm{C}$ & -7.23069500 & 2.10640400 & 0.49079100 & \\
\hline $\mathrm{C}$ & -6.31888500 & -3.92109900 & -0.46645500 & \\
\hline 0 & -3.60573800 & 3.39282100 & -0.99762900 & \\
\hline 0 & -2.40602500 & -3.69445000 & -1.74225600 & \\
\hline $\mathrm{C}$ & -3.44527700 & -0.25025000 & -1.05698200 & \\
\hline $\mathrm{C}$ & -3.85431000 & 2.22077400 & -0.81182000 & \\
\hline $\mathrm{C}$ & -3.04520800 & -2.70973700 & -1.43021700 & \\
\hline 0 & -5.03324200 & 1.78551300 & -0.34741600 & \\
\hline 0 & -4.33367100 & -2.76539800 & -1.05804500 & \\
\hline $\mathrm{C}$ & -2.92956900 & 1.08623700 & -1.05253900 & \\
\hline $\mathrm{C}$ & -1.57262500 & 1.30001500 & -1.14589100 & \\
\hline & & -132080100 & -138637000 & \\
\hline
\end{tabular}




\begin{tabular}{|c|c|c|c|}
\hline & & -1.06078600 & \\
\hline & & & \\
\hline & -3.66507500 & & \\
\hline & 0.18061100 & 2.46248800 & \\
\hline & -0.89958300 & & \\
\hline & 741700 & & \\
\hline & -6.21 & & \\
\hline & 604700 & 3.473 & \\
\hline & -0.36 & & \\
\hline & 0.83 & -1.5 & \\
\hline & -0.0 & & \\
\hline & -7.6 & 1.43 & \\
\hline & -6.9 & & \\
\hline & -7.9 & & \\
\hline & -4.2 & -4.7 & \\
\hline & -4.9 & -4.51 & \\
\hline & -6.3 & -3.47 & \\
\hline & -6.9 & -3.2 & \\
\hline & -6.8 & -4.90 & \\
\hline & -0.4 & & \\
\hline & -1.1 & -1.87 & \\
\hline & -2.5 & & \\
\hline & -3.3 & -0.7 & \\
\hline & -2.5 & & \\
\hline & -3.2 & & \\
\hline & -2.5 & & \\
\hline & -1.1 & & \\
\hline & -0.4 & & \\
\hline & -1.1 & & \\
\hline & -0.6 & -2.7 & \\
\hline & -3.0 & -2.9 & \\
\hline & -4.3 & -0.81 & \\
\hline & -4.3 & & \\
\hline & -3.0 & & \\
\hline & -0.5 & & \\
\hline & 0.6 & 1.58 & \\
\hline & -4.50 & -0.38 & \\
\hline & & -1.28 & \\
\hline & 5.86694600 & -2.36 & -0.0 \\
\hline & & -2.45 & \\
\hline & 4.05 & -1.455 & 500 \\
\hline & & -0.73 & \\
\hline & 5.08151500 & 0.51886900 & 1.95669700 \\
\hline & & & \\
\hline & 2.01162200 & -1.03507000 & 1.25422200 \\
\hline & 4.34302700 & 0.45407000 & 2.75629100 \\
\hline
\end{tabular}




\begin{tabular}{|c|c|c|c|c|}
\hline $\mathrm{H}$ & 4.82480100 & 1.32755800 & 1.26953600 & \\
\hline $\mathrm{H}$ & 6.06750000 & 0.69446800 & 2.38649300 & \\
\hline $\mathrm{H}$ & 6.43014200 & -3.08079100 & $-0.6314350 c$ & \\
\hline $\mathrm{H}$ & 7.23304800 & -0.85179800 & 0.83356300 & \\
\hline $\mathrm{H}$ & 4.30685900 & -4.19856700 & -1.03185600 & \\
\hline $\mathrm{H}$ & 3.06169100 & -2.96387500 & $-1.3589360 c$ & \\
\hline $\mathrm{C}$ & 3.06947300 & 2.67477400 & -0.80646700 & \\
\hline $\mathrm{S}$ & 3.25132400 & 1.28395700 & -1.98497300 & \\
\hline $\mathrm{F}$ & 2.35510900 & 3.66418100 & -1.34360600 & \\
\hline $\mathrm{F}$ & 4.26389700 & 3.15944200 & -0.46059300 & \\
\hline $\mathrm{F}$ & 2.44754800 & 2.27923800 & 0.31120800 & \\
\hline 0 & 4.11666700 & 0.34498700 & -1.26332500 & \\
\hline 0 & 1.85536300 & 0.83236600 & -2.16072700 & \\
\hline 0 & 3.83784100 & 1.91410900 & -3.16636400 & \\
\hline $\mathrm{H}$ & 0.23961200 & 0.39625700 & -1.45667000 & \\
\hline $\mathrm{H}$ & 3.00043700 & -3.92679300 & 0.14723200 & \\
\hline \multicolumn{3}{|c|}{ Zero-point correction $=$} & 0.6117 & 60 (Hartree/Particle) \\
\hline \multicolumn{3}{|c|}{ Thermal correction to Energy= } & & 57625 \\
\hline \multicolumn{3}{|c|}{ Thermal correction to Enthalpy= } & & 558570 \\
\hline \multicolumn{4}{|c|}{ Thermal correction to Gibbs Free Energy= } & 0.528156 \\
\hline \multicolumn{4}{|c|}{ Sum of electronic and zero-point Energies= } & -2824.850816 \\
\hline \multicolumn{4}{|c|}{ Sum of electronic and thermal Energies $=$} & -2824.804951 \\
\hline \multicolumn{4}{|c|}{ Sum of electronic and thermal Enthalpies $=$} & -2824.804006 \\
\hline \multicolumn{5}{|c|}{ Sum of electronic and thermal Free Energies $=$} \\
\hline
\end{tabular}

\begin{tabular}{lrrr}
\multicolumn{4}{c}{ C4-XB-TS1BD } \\
$\mathrm{C}$ & 6.90967300 & -2.64792800 & 0.73842200 \\
$\mathrm{C}$ & 5.45015400 & 4.13066800 & -0.21635600 \\
$\mathrm{C}$ & 2.11636600 & -2.84036000 & -1.24906500 \\
$\mathrm{C}$ & 1.05124700 & 1.76325600 & -1.94684900 \\
$\mathrm{C}$ & 8.03235100 & -1.90055100 & 1.42419700 \\
$\mathrm{C}$ & 6.65254400 & 4.11932900 & 0.70177700 \\
0 & 4.70199500 & -3.38014200 & -0.45134600 \\
0 & 3.33677100 & 3.55433900 & -1.62631300 \\
$\mathrm{C}$ & 4.15983000 & 0.28203100 & -0.06714900 \\
$\mathrm{C}$ & 4.77990800 & -2.19123600 & -0.17447400 \\
$\mathrm{C}$ & 3.77474400 & 2.63597600 & -0.95006400 \\
0 & 5.87758800 & -1.70037800 & 0.45795100 \\
0 & 4.86796500 & 2.82546000 & -0.16917500 \\
$\mathrm{C}$ & 3.80217200 & -1.14174200 & -0.43215700 \\
$\mathrm{C}$ & 2.56133700 & -1.43024300 & -0.94289200 \\
$\mathrm{C}$ & 3.28673400 & 1.25758300 & -0.83251900 \\
$\mathrm{C}$ & 2.06987000 & 0.84745600 & -1.31683400 \\
$\mathrm{~N}$ & 1.63182200 & -0.46234300 & -1.24776200 \\
$\mathrm{H}$ & 4.07804800 & 0.44961300 & 1.02178900 \\
$\mathrm{H}$ & 1.05476600 & -2.84925700 & -1.50221900
\end{tabular}




\begin{tabular}{|c|c|c|c|}
\hline & & & \\
\hline & & -3.52107600 & \\
\hline & 24307000 & -3.10783100 & \\
\hline & & & \\
\hline & 81518600 & & \\
\hline & & & \\
\hline & 39500900 & 2.79157900 & \\
\hline & 42065900 & & \\
\hline & & & \\
\hline & 6800 & -2.5 & \\
\hline & & & \\
\hline & 5.73079 & & \\
\hline & & & \\
\hline & & & \\
\hline & & & \\
\hline & 700 & & \\
\hline & & & \\
\hline & & & \\
\hline & & & \\
\hline & & & \\
\hline & & & \\
\hline & & & \\
\hline & 0 & -3.2 & \\
\hline & & -2.2 & \\
\hline & -0.22693 & & \\
\hline & & & \\
\hline & -0.571005 & & \\
\hline & -1.6166610 & & \\
\hline & -2.07 & & \\
\hline & -1.7976330 & -3.7 & \\
\hline & -0.487205 & -4.3 & \\
\hline & 0.52509000 & -2.5 & \\
\hline & 0.7899 & & \\
\hline & 5.212189 & & \\
\hline & -2.879308 & -0. & \\
\hline & -4.55214900 & & \\
\hline & -3.66 & & \\
\hline & -3.32308300 & & -3.4 \\
\hline & -4.02282400 & & \\
\hline & -1.12450400 & -1.006 & -1.77 \\
\hline & -6.15332300 & & \\
\hline & & -1.2 & -2.4 \\
\hline & -3.17223100 & -1.35810400 & -1.8 \\
\hline & & -1.96672100 & \\
\hline & -3.88449500 & -2.02791900 & -0.94517600 \\
\hline & -3.89934900 & 1.35577600 & -0.99091400 \\
\hline
\end{tabular}




\begin{tabular}{llll}
$\mathrm{H}$ & -2.61602500 & -3.44793800 & -0.06698500 \\
$\mathrm{C}$ & -3.33429000 & -2.68425400 & 0.23835000 \\
0 & -1.81938500 & 2.32484300 & -0.04220600 \\
$\mathrm{H}$ & -5.97496500 & -2.44538500 & -0.68195400 \\
$\mathrm{~S}$ & -3.03350500 & 1.52708100 & 0.18412800 \\
$\mathrm{H}$ & -4.16041600 & -3.15204100 & 0.77336300 \\
$\mathrm{H}$ & -2.85682900 & -1.92883600 & 0.86525900 \\
0 & -2.84204800 & 0.31719600 & 0.99897700 \\
$\mathrm{~F}$ & -4.29164900 & 3.76839600 & 0.70308200 \\
$\mathrm{C}$ & -4.03166700 & 2.59716900 & 1.28532900 \\
$\mathrm{~F}$ & -5.19375000 & 2.01251900 & 1.58392100 \\
$\mathrm{~F}$ & -3.38280200 & 2.83522800 & 2.42866000 \\
Zero-point correction= & 0.615160 (Hartree/Particle) \\
Thermal correction to Energy= & 0.661295 \\
Thermal correction to Enthalpy= & \multicolumn{2}{c}{0.662239} \\
Thermal correction to Gibbs Free Energy= & 0.528835 \\
Sum of electronic and zero-point Energies= & -2824.853787 \\
Sum of electronic and thermal Energies= & -2824.807653 \\
Sum of electronic and thermal Enthalpies= & -2824.806708 \\
Sum of electronic and thermal Free Energies= & -2824.940113
\end{tabular}

\begin{tabular}{lrrr}
\multicolumn{4}{l}{ C4-XB-TS2B } \\
$\mathrm{C}$ & 6.47327300 & 1.96312000 & -0.71680600 \\
$\mathrm{C}$ & 4.38931300 & -4.52946000 & 0.52891100 \\
$\mathrm{C}$ & 1.87028400 & 2.77033300 & 1.59255900 \\
$\mathrm{C}$ & 0.26814200 & -1.71013000 & 2.26769100 \\
$\mathrm{C}$ & 7.45092400 & 1.04199900 & -1.41155700 \\
$\mathrm{C}$ & 5.69374000 & -4.59049700 & -0.23403900 \\
0 & 4.44356200 & 3.01569400 & 0.57167500 \\
0 & 2.15904800 & -3.79303100 & 1.68528200 \\
$\mathrm{C}$ & 3.65539600 & -0.56323300 & 0.77894400 \\
$\mathrm{C}$ & 4.43964200 & 1.80292200 & 0.46647000 \\
$\mathrm{C}$ & 2.87139100 & -2.88758400 & 1.28823300 \\
0 & 5.44313600 & 1.14276600 & -0.15534600 \\
0 & 4.05953700 & -3.14597900 & 0.69179600 \\
$\mathrm{C}$ & 3.40857100 & 0.86581200 & 0.93485300 \\
$\mathrm{C}$ & 2.18557600 & 1.30723200 & 1.41546400 \\
$\mathrm{C}$ & 2.62749500 & -1.43803600 & 1.34300100 \\
$\mathrm{C}$ & 1.42984800 & -0.88552100 & 1.78211000 \\
$\mathrm{~N}$ & 1.20333500 & 0.45008700 & 1.79124700 \\
$\mathrm{H}$ & 3.63598600 & -0.79755000 & -0.45921100 \\
$\mathrm{H}$ & 0.90349100 & 2.88156300 & 2.08540200 \\
$\mathrm{H}$ & 2.63740600 & 3.26250400 & 2.19393900 \\
$\mathrm{H}$ & 1.85113900 & 3.28971800 & 0.62900300 \\
$\mathrm{H}$ & 6.95219300 & 2.53652400 & 0.08288400 \\
$\mathrm{H}$ & 6.01934700 & 2.67629500 & -1.41341700
\end{tabular}




\begin{tabular}{|c|c|c|c|}
\hline & & & \\
\hline & & & \\
\hline & -0.39996200 & & \\
\hline & 7.88682500 & & \\
\hline & 6.95366700 & & \\
\hline & 8.25922200 & & \\
\hline & & & \\
\hline & 4.47185000 & -4.9 & \\
\hline & & & \\
\hline & 6.488 & -4.0 & \\
\hline & & -5.6 & \\
\hline & 0.39 & -0.5 & \\
\hline & & & \\
\hline & 2.202 & -2.0 & \\
\hline & & -1.0 & \\
\hline & & & \\
\hline & 3.27 & & -2.4 \\
\hline & & & -2.5 \\
\hline & & 2.8 & -2.2 \\
\hline & & & \\
\hline & & & -1.7 \\
\hline & & & \\
\hline & & -3.1 & \\
\hline & & -1.1 & -2.2 \\
\hline & & & -2.6 \\
\hline & & & -2.8 \\
\hline & & & -2.3 \\
\hline & -0.4 & & -1.6 \\
\hline & -0.5 & -0.4 & -1.1 \\
\hline & & -0.8 & \\
\hline & & & \\
\hline & & & \\
\hline & -3.9 & & \\
\hline & -3.4 & & \\
\hline & -4.4 & & \\
\hline & -4.4 & & \\
\hline & -3.0 & & -0.8 \\
\hline & -1.45 & 1.25 & 1.46 \\
\hline & -3.7 & & \\
\hline & & & \\
\hline & -5.4 & 0.2 & 4600 \\
\hline & -5.8 & & -0.50 \\
\hline & -6.62165900 & 1.57077900 & 09000 \\
\hline & -2.73850400 & & \\
\hline & -2.24857300 & 3.73487900 & -0.39662900 \\
\hline & -3.86909600 & -1.88972200 & -2.45488200 \\
\hline
\end{tabular}




\begin{tabular}{llrc}
$\mathrm{S}$ & -3.11145900 & -1.00704600 & -1.04176900 \\
$\mathrm{~F}$ & -4.79051700 & -1.12542900 & -3.04067000 \\
$\mathrm{~F}$ & -4.45191300 & -3.01468600 & -2.04367800 \\
$\mathrm{~F}$ & -2.94186400 & -2.20072800 & -3.35988200 \\
0 & -2.09953300 & -1.95486600 & -0.54568900 \\
0 & -2.52243200 & 0.19179400 & -1.67274200 \\
0 & -4.24221400 & -0.75286200 & -0.14525300 \\
$\mathrm{H}$ & -3.71627200 & 3.97208100 & -1.37536700 \\
Zero-point correction= & \multicolumn{3}{c}{0.611503 (Hartree/Particle) } \\
Thermal correction to Energy= & 0.657298 \\
Thermal correction to Enthalpy= & 0.658242 \\
Thermal correction to Gibbs Free Energy $=$ & 0.527641 \\
Sum of electronic and zero-point Energies $=$ & -2824.856153 \\
Sum of electronic and thermal Energies $=$ & -2824.810358 \\
Sum of electronic and thermal Enthalpies $=$ & -2824.809414 \\
Sum of electronic and thermal Free Energies $=$ & -2824.940015
\end{tabular}

\begin{tabular}{|c|c|c|c|}
\hline \multicolumn{4}{|c|}{ C4-XB-TS3G } \\
\hline & 6.57607000 & 2.83924000 & -0.4899300 \\
\hline & 4.08353800 & & \\
\hline & 1.57554300 & & 92078300 \\
\hline & -0.14923700 & -0.96030600 & 0.87666000 \\
\hline & 7.77722800 & & \\
\hline & 5.58757100 & & \\
\hline & 0.35365600 & 0.14901500 & 3160700 \\
\hline & 4.19260300 & 3.34690900 & \\
\hline & 1.61 & -2.06 & \\
\hline & & & \\
\hline & & & \\
\hline & 2.48 & -1.4 & \\
\hline & & & \\
\hline & $3.7 \mathrm{~s}$ & -1.6 & \\
\hline & 3.19 & & -0.2 \\
\hline & 1.94985200 & & \\
\hline & 2.30511200 & & \\
\hline & & & \\
\hline & 0.89 & & -0. \\
\hline & 4.31 & -0.2 & \\
\hline & 3.97 & & 34500 \\
\hline & & & \\
\hline & & & \\
\hline & & & \\
\hline & 6.36633800 & 3.86991800 & -0.18709500 \\
\hline & 6.71876100 & 2.83344100 & -1.57519900 \\
\hline & & & \\
\hline & 7.96866500 & 1.24442900 & -0.0766070 \\
\hline
\end{tabular}




\begin{tabular}{|c|c|c|c|}
\hline & & & \\
\hline & & & \\
\hline & 3.67297700 & & \\
\hline & & & \\
\hline & & & \\
\hline & & & \\
\hline & & & \\
\hline & & & \\
\hline & & & \\
\hline & 3.25090 & -3.6 & \\
\hline & & & \\
\hline & & -2.7 & \\
\hline & & & \\
\hline & 0.19942 & -0.4 & \\
\hline & & & \\
\hline & & -2.6 & \\
\hline & 5.1 & -2.7 & \\
\hline & & -4.3 & \\
\hline & 1.20 & -4.3 & -0 . \\
\hline & 4.25 & & \\
\hline & -0.620 & & -1. \\
\hline & & & \\
\hline & -0.535 & & \\
\hline & 0.046 & -2.0 & \\
\hline & & -1.2 & \\
\hline & 2.2 & & -3.2 \\
\hline & -1.650 & -1.5 & -2. \\
\hline & -0.340 & & -3. \\
\hline & -4.8 & & \\
\hline & & & \\
\hline & -4.295 & & \\
\hline & -6.2567 & & \\
\hline & -3.2506 & & \\
\hline & -3.5868 & & 900 \\
\hline & -5.3478 & & \\
\hline & -2.72892 & 5.4 & 100 \\
\hline & -4.33406 & & \\
\hline & -1.43344 & 2166 & 00 \\
\hline & -5.34137100 & 0.68675800 & \\
\hline & -3.61486 & & \\
\hline & -2.52190600 & 5.24786100 & 1500 \\
\hline & -1.79629900 & & \\
\hline 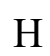 & -3.25687300 & 6.35421200 & 0.21595200 \\
\hline & -4.99388700 & -2.99898400 & \\
\hline & -3.30883400 & -2.33136300 & -0.09337100 \\
\hline & -5.17195200 & -4.11209500 & 0.37150500 \\
\hline
\end{tabular}




$\begin{array}{lrrr}\mathrm{F} & -5.21101900 & -3.28051800 & -1.62306700 \\ \mathrm{~F} & -5.90072700 & -2.10065800 & 0.05296000 \\ 0 & -3.31082300 & -1.12836100 & -0.94605400 \\ 0 & -3.25206600 & -2.07483100 & 1.34704800 \\ 0 & -2.44096300 & -3.41732800 & -0.58519700 \\ \mathrm{H} & -4.09043800 & 0.39635100 & 0.51767800 \\ \text { Zero-point correction }= & 0.634208 \text { (Hartree/Particle) } \\ \text { Thermal correction to Energy }= & 0.680680 \\ \text { Thermal correction to Enthalpy= } & 0.681624 \\ \text { Thermal correction to Gibbs Free Energy= } & 0.547813 \\ \text { Sum of electronic and zero-point Energies }= & -2826.011275 \\ \text { Sum of electronic and thermal Energies }= & -2825.964803 \\ \text { Sum of electronic and thermal Enthalpies }= & -2825.963859 \\ \text { Sum of electronic and thermal Free Energies }= & -2826.097670\end{array}$

\begin{tabular}{lrrr}
\multicolumn{4}{l}{ C4-XB-TS4G } \\
$\mathrm{C}$ & -4.64412600 & -1.33036800 & 2.05834800 \\
$\mathrm{C}$ & -3.93053500 & 4.13209400 & -2.32188000 \\
$\mathrm{C}$ & 0.12164700 & 0.46701900 & 3.26767200 \\
$\mathrm{C}$ & 0.34403600 & 4.49476600 & 0.67417700 \\
$\mathrm{C}$ & -5.99831800 & -1.13923300 & 1.41236000 \\
$\mathrm{C}$ & -5.11226100 & 3.34722100 & -2.84690800 \\
0 & -2.22521900 & -1.02822200 & 3.01392200 \\
0 & -1.82674700 & 4.99824200 & -1.01026000 \\
$\mathrm{C}$ & -2.33287700 & 1.67970500 & 0.51387300 \\
$\mathrm{C}$ & -2.56439600 & -0.21833200 & 2.16646100 \\
$\mathrm{C}$ & -2.21497800 & 3.88050600 & -0.71621900 \\
0 & -3.78354300 & -0.29345600 & 1.57368400 \\
0 & -3.27936400 & 3.31780600 & -1.34048600 \\
$\mathrm{C}$ & -1.79867800 & 0.92180800 & 1.66327300 \\
$\mathrm{C}$ & -0.56488900 & 1.27156400 & 2.19489800 \\
$\mathrm{C}$ & -1.64635800 & 2.96934800 & 0.27714500 \\
$\mathrm{C}$ & -0.44837000 & 3.23302300 & 0.90740500 \\
$\mathrm{~N}$ & 0.11752800 & 2.37797900 & 1.80655000 \\
$\mathrm{H}$ & -2.17895300 & 1.00147800 & -0.44858300 \\
$\mathrm{H}$ & -3.42250000 & 1.75603200 & 0.50103300 \\
$\mathrm{H}$ & 0.38453900 & -0.52733100 & 2.89238600 \\
$\mathrm{H}$ & 1.02810500 & 0.99006100 & 3.58144500 \\
$\mathrm{H}$ & -0.53160300 & 0.31816100 & 4.13050100 \\
$\mathrm{H}$ & -4.70924600 & -1.25747500 & 3.14832100 \\
$\mathrm{H}$ & -4.20731100 & -2.30329500 & 1.81278700 \\
$\mathrm{H}$ & -0.25255600 & 5.38013400 & 0.90728100 \\
$\mathrm{H}$ & 1.23306200 & 4.48151800 & 1.30859700 \\
$\mathrm{H}$ & 0.64397700 & 4.59202100 & -0.37408700 \\
$\mathrm{H}$ & -6.42257000 & -0.16794700 & 1.68405100 \\
$\mathrm{H}$ & -5.92534600 & -1.19555100 & 0.32223900
\end{tabular}




\begin{tabular}{|c|c|c|c|}
\hline & -6.68187700 & & \\
\hline & & & \\
\hline & -4.24457200 & & \\
\hline & & & \\
\hline & -5.80284200 & & \\
\hline & -5.65009400 & & \\
\hline & -1.35585600 & -1.17090000 & \\
\hline & -1.50923300 & & \\
\hline & -2.573 & -0.2 & \\
\hline & -3.83 & & \\
\hline & -3.50 & & \\
\hline & -4.37 & -3.2 & \\
\hline & -4.0 & & \\
\hline & -2.82 & -4.3 & \\
\hline & -1.9 & & \\
\hline & -2.27 & -2.2 & \\
\hline & & & \\
\hline & -4.73 & -5.1 & \\
\hline & -2.56 & -5.2 & \\
\hline & -0.97 & -3.3 & \\
\hline & $-0.4 \mathrm{C}$ & -1.2 & \\
\hline & -0.62 & & \\
\hline & -2.16 & -0.8 & \\
\hline & $-2.7^{\prime}$ & & \\
\hline & -4.5 & & \\
\hline & -4.3 & -0.1 & \\
\hline & & & \\
\hline & 6.11 & -0.2 & \\
\hline & 5.26 & & \\
\hline & 4.22 & & \\
\hline & 4.39 & & \\
\hline & 3.47 & & \\
\hline & & & \\
\hline & 2.53 & 1.59 & $0.7^{\prime}$ \\
\hline & 3.36 & & \\
\hline & 2.51 & & -2.4 \\
\hline & 3.90 & & -3.5 \\
\hline & 7.019 & -0.74013100 & -0.3 \\
\hline & 5.91 & 0.03 & -2.8 \\
\hline & & -0.48683400 & \\
\hline & 4.62 & -0.76083300 & \\
\hline & & -3.59 & \\
\hline & 2.050 & -1.92488600 & -0.3 \\
\hline & 2.54867200 & & \\
\hline & 0.46236500 & -3.64591600 & -1.45352100 \\
\hline & 1.83920400 & -4.51974200 & -0.03279200 \\
\hline
\end{tabular}




$\begin{array}{lrrr}0 & 1.07841600 & -1.82673200 & 0.80454700 \\ 0 & 3.45443900 & -1.98682000 & 0.10552600 \\ 0 & 1.74935500 & -1.04331700 & -1.44473300 \\ \mathrm{H} & 5.30856700 & 0.87201300 & 2.35317100 \\ \text { Zero-point correction }= & 0.635700 \text { (Hartree/Particle) } \\ \text { Thermal correction to Energy }= & 0.682125 \\ \text { Thermal correction to Enthalpy= } & 0.683069 \\ \text { Thermal correction to Gibbs Free Energy }= & 0.549606 \\ \text { Sum of electronic and zero-point Energies }= & -2826.027754 \\ \text { Sum of electronic and thermal Energies }= & -2825.981329 \\ \text { Sum of electronic and thermal Enthalpies }= & -2825.980385 \\ \text { Sum of electronic and thermal Free Energies }= & -2826.113848\end{array}$

\begin{tabular}{|c|c|c|c|c|}
\hline \multicolumn{5}{|c|}{ EN-QNL } \\
\hline $\mathrm{N}$ & 2.86780500 & -0.80037400 & \multicolumn{2}{|l|}{-0.11618300} \\
\hline $\mathrm{C}$ & 3.16610400 & -2.00100400 & \multicolumn{2}{|l|}{0.52444700} \\
\hline $\mathrm{C}$ & 4.42006800 & -2.40175800 & \multicolumn{2}{|l|}{0.75337400} \\
\hline $\mathrm{C}$ & 5.60068800 & -1.58558100 & \multicolumn{2}{|l|}{0.29146500} \\
\hline $\mathrm{C}$ & 5.19769000 & -0.15044500 & \multicolumn{2}{|l|}{0.00351600} \\
\hline $\mathrm{C}$ & 6.15319600 & 0.86197400 & \multicolumn{2}{|l|}{-0.07654500} \\
\hline $\mathrm{C}$ & 5.80734200 & 2.17487600 & \multicolumn{2}{|l|}{-0.38840100} \\
\hline $\mathrm{C}$ & 4.46849100 & 2.48906100 & \multicolumn{2}{|l|}{-0.61939200} \\
\hline $\mathrm{C}$ & 3.49425800 & 1.50276700 & \multicolumn{2}{|l|}{-0.52854200} \\
\hline $\mathrm{C}$ & 3.84989900 & 0.18437600 & \multicolumn{2}{|l|}{-0.21743800} \\
\hline $\mathrm{H}$ & 7.19511100 & 0.60591400 & \multicolumn{2}{|l|}{0.10447100} \\
\hline $\mathrm{H}$ & 6.57251200 & 2.94207400 & \multicolumn{2}{|l|}{-0.45148000} \\
\hline $\mathrm{H}$ & 4.17785200 & 3.50676300 & \multicolumn{2}{|l|}{-0.86301400} \\
\hline $\mathrm{H}$ & 2.44647800 & 1.74240600 & \multicolumn{2}{|l|}{-0.69323100} \\
\hline $\mathrm{H}$ & 1.91100300 & -0.47597100 & \multicolumn{2}{|l|}{-0.06136200} \\
\hline $\mathrm{H}$ & 2.29991800 & -2.58730600 & \multicolumn{2}{|l|}{0.81199500} \\
\hline $\mathrm{H}$ & 6.39833100 & -1.59680000 & \multicolumn{2}{|l|}{1.04462700} \\
\hline $\mathrm{H}$ & 6.04365200 & -2.03389700 & \multicolumn{2}{|l|}{-0.61183800} \\
\hline $\mathrm{H}$ & 4.59305200 & -3.35036100 & \multicolumn{2}{|l|}{1.24953700} \\
\hline \multicolumn{5}{|c|}{ Zero-point correction $=$} \\
\hline \multicolumn{5}{|c|}{ Thermal correction to Energy= } \\
\hline \multicolumn{5}{|c|}{ Thermal correction to Enthalpy $=\quad 0.168575$} \\
\hline \multicolumn{5}{|c|}{ Thermal correction to Gibbs Free Energy $=\quad 0.127837$} \\
\hline \multicolumn{5}{|c|}{ Sum of electronic and zero-point Energies $=$} \\
\hline \multicolumn{5}{|c|}{ Sum of electronic and thermal Energies $=$} \\
\hline \multicolumn{5}{|c|}{ Sum of electronic and thermal Enthalpies= } \\
\hline \multicolumn{5}{|c|}{ Sum of electronic and thermal Free Energies= } \\
\hline \multicolumn{5}{|c|}{ HEH } \\
\hline $\mathrm{C}$ & 3.64469400 & -2.60630000 & 0.63696700 & \\
\hline $\mathrm{C}$ & -3.36441900 & -2.56184500 & 2.18435200 & \\
\hline & & 2.29713 & & \\
\hline
\end{tabular}




\begin{tabular}{|c|c|c|c|c|}
\hline $\mathrm{C}$ & -2.47825100 & 2.32701300 & 0.56579300 & \\
\hline $\mathrm{C}$ & 3.32317700 & -4.00446900 & 1.11435200 & \\
\hline $\mathrm{C}$ & -2.88861500 & -3.96495600 & 2.48606000 & \\
\hline $\mathrm{H}$ & -0.16867500 & 3.01736000 & -0.14919300 & \\
\hline 0 & 3.57403200 & -0.07935300 & -0.08175800 & \\
\hline 0 & -3.57242500 & -0.03405600 & 1.49568200 & \\
\hline $\mathrm{C}$ & 0.03605100 & -0.69682900 & 0.88506100 & \\
\hline $\mathrm{C}$ & 2.51926200 & -0.56210300 & 0.29321800 & \\
\hline $\mathrm{C}$ & -2.46371000 & -0.53048700 & 1.39324900 & \\
\hline 0 & 2.42809100 & -1.84907000 & 0.68768000 & \\
\hline 0 & -2.23048500 & -1.81947700 & 1.71615600 & \\
\hline $\mathrm{C}$ & 1.21911300 & 0.11051100 & 0.38695900 & \\
\hline $\mathrm{C}$ & 1.10947700 & 1.41577900 & 0.03193200 & \\
\hline $\mathrm{C}$ & -1.23687100 & 0.12612600 & 0.92917300 & \\
\hline $\mathrm{C}$ & -1.27109500 & 1.43091500 & 0.55753200 & \\
\hline $\mathrm{N}$ & -0.11500500 & 2.04427400 & 0.12190800 & \\
\hline $\mathrm{H}$ & -0.10979700 & -1.58051600 & 0.24989900 & \\
\hline $\mathrm{H}$ & 0.25317700 & -1.10645900 & 1.88037700 & \\
\hline $\mathrm{H}$ & 2.65763100 & 1.88461500 & -1.38177900 & \\
\hline $\mathrm{H}$ & 1.84200100 & 3.30164200 & -0.67977500 & \\
\hline $\mathrm{H}$ & 3.02335500 & 2.36363700 & 0.26383600 & \\
\hline $\mathrm{H}$ & 4.39277200 & -2.12137700 & 1.27162900 & \\
\hline $\mathrm{H}$ & 4.02339100 & -2.60369700 & -0.38974500 & \\
\hline $\mathrm{H}$ & -2.89817300 & 2.40087500 & 1.57092600 & \\
\hline $\mathrm{H}$ & -2.21210600 & 3.32756800 & 0.21577200 & \\
\hline $\mathrm{H}$ & -3.26449000 & 1.92257900 & -0.07477800 & \\
\hline $\mathrm{H}$ & 2.94246400 & -3.98511300 & 2.13947700 & \\
\hline $\mathrm{H}$ & 2.57026000 & -4.46896100 & 0.47125500 & \\
\hline $\mathrm{H}$ & 4.22681900 & -4.62011300 & 1.09128600 & \\
\hline $\mathrm{H}$ & -4.13998200 & -2.55212700 & 1.41236700 & \\
\hline $\mathrm{H}$ & -3.77003100 & -2.06949700 & 3.07352600 & \\
\hline $\mathrm{H}$ & -2.48248500 & -4.43719700 & 1.58703300 & \\
\hline $\mathrm{H}$ & -2.11116900 & -3.95259800 & 3.25523500 & \\
\hline $\mathrm{H}$ & -3.72519400 & -4.56940300 & 2.84785800 & \\
\hline \multicolumn{3}{|c|}{ Zero-point correction $=$} & 0.31423 & 6 (Hartree/Particle) \\
\hline \multicolumn{3}{|c|}{ Thermal correction to Energy= } & 0.33 & 3819 \\
\hline \multicolumn{3}{|c|}{ Thermal correction to Enthalpy= } & 0.3 & 34763 \\
\hline \multicolumn{4}{|c|}{ Thermal correction to Gibbs Free Energy= } & 0.264950 \\
\hline \multicolumn{4}{|c|}{ Sum of electronic and zero-point Energies $=$} & -861.839491 \\
\hline \multicolumn{4}{|c|}{ Sum of electronic and thermal Energies $=$} & -861.819908 \\
\hline \multicolumn{4}{|c|}{ Sum of electronic and thermal Enthalpies $=$} & -861.818964 \\
\hline \multicolumn{4}{|c|}{ Sum of electronic and thermal Free Energies= } & -861.888777 \\
\hline \multicolumn{5}{|c|}{ HEH-RADCAT } \\
\hline & 3.55034300 & 2.11870400 & 0.00007500 & \\
\hline $\mathrm{C}$ & -3.55026300 & 2.11881600 & 0.00001300 & \\
\hline
\end{tabular}




\begin{tabular}{|c|c|c|c|c|}
\hline $\mathrm{C}$ & 2.40899400 & -3.03777900 & 0.00005600 & \\
\hline $\mathrm{C}$ & -2.40907600 & -3.03769700 & 0.00000500 & \\
\hline $\mathrm{C}$ & 3.10197600 & 3.56038700 & 0.00023100 & \\
\hline $\mathrm{C}$ & -3.10185500 & 3.56048700 & 0.00009400 & \\
\hline $\mathrm{H}$ & -0.00005500 & -3.74192600 & 0.00007100 & \\
\hline 0 & 3.64106500 & -0.54573800 & -0.00018100 & \\
\hline 0 & -3.64106400 & -0.54562000 & -0.00002300 & \\
\hline $\mathrm{C}$ & 0.00001000 & 0.04662200 & -0.00009200 & \\
\hline $\mathrm{C}$ & 2.55263300 & -0.01473100 & -0.00010500 & \\
\hline $\mathrm{C}$ & -2.55261500 & -0.01464700 & -0.00007100 & \\
\hline 0 & 2.35803600 & 1.30083000 & 0.00004200 & \\
\hline 0 & -2.35797900 & 1.30090800 & -0.00005600 & \\
\hline $\mathrm{C}$ & 1.25199000 & -0.74413400 & -0.00006800 & \\
\hline $\mathrm{C}$ & 1.22697600 & -2.11942400 & -0.00000100 & \\
\hline $\mathrm{C}$ & -1.25199600 & -0.74409100 & -0.00006700 & \\
\hline $\mathrm{C}$ & -1.22702900 & -2.11938000 & -0.00002000 & \\
\hline $\mathrm{N}$ & -0.00003600 & -2.72110200 & 0.00001500 & \\
\hline $\mathrm{H}$ & 0.00002500 & 0.74679100 & 0.85546000 & \\
\hline $\mathrm{H}$ & 0.00002000 & 0.74674600 & -0.85567900 & \\
\hline $\mathrm{H}$ & 3.02964800 & -2.85128800 & 0.87876600 & \\
\hline $\mathrm{H}$ & 2.08606800 & -4.08069600 & 0.00025400 & \\
\hline $\mathrm{H}$ & 3.02949800 & -2.85158200 & -0.87882500 & \\
\hline $\mathrm{H}$ & 4.13828700 & 1.86785200 & -0.88685000 & \\
\hline $\mathrm{H}$ & 4.13835700 & 1.86768200 & 0.88690600 & \\
\hline $\mathrm{H}$ & -3.02965800 & -2.85134700 & -0.87878800 & \\
\hline $\mathrm{H}$ & -2.08618200 & -4.08062300 & 0.00001800 & \\
\hline $\mathrm{H}$ & -3.02964200 & -2.85132000 & 0.87880300 & \\
\hline $\mathrm{H}$ & 2.50607200 & 3.78203000 & -0.88930100 & \\
\hline $\mathrm{H}$ & 2.50611900 & 3.78185000 & 0.88984100 & \\
\hline $\mathrm{H}$ & 3.97999700 & 4.21186800 & 0.00027500 & \\
\hline $\mathrm{H}$ & -4.13823500 & 1.86784200 & 0.88688400 & \\
\hline $\mathrm{H}$ & -4.13826200 & 1.86794900 & -0.88687100 & \\
\hline $\mathrm{H}$ & -2.50595600 & 3.78196900 & 0.88967000 & \\
\hline $\mathrm{H}$ & -2.50598100 & 3.78207800 & -0.88947200 & \\
\hline $\mathrm{H}$ & -3.97985800 & 4.21199100 & 0.00014600 & \\
\hline \multicolumn{3}{|c|}{ Zero-point correction $=$} & 0.31208 & 31 (Hartree/Particle) \\
\hline \multicolumn{3}{|c|}{ Thermal correction to Energy= } & 0.33 & 0919 \\
\hline \multicolumn{3}{|c|}{ Thermal correction to Enthalpy= } & 0.3 & 31863 \\
\hline \multicolumn{4}{|c|}{ Thermal correction to Gibbs Free Energy= } & 0.263766 \\
\hline \multicolumn{4}{|c|}{ Sum of electronic and zero-point Energies $=$} & -861.643638 \\
\hline \multicolumn{4}{|c|}{ Sum of electronic and thermal Energies $=$} & -861.624801 \\
\hline \multicolumn{4}{|c|}{ Sum of electronic and thermal Enthalpies= } & -861.623857 \\
\hline \multicolumn{5}{|c|}{ Sum of electronic and thermal Free Energies $=$} \\
\hline
\end{tabular}

\section{IM-QNL}

H $\quad 1.24036400 \quad 2.47959900 \quad-0.04207400$ 


\begin{tabular}{|c|c|c|c|}
\hline & & & \\
\hline & & & \\
\hline & & & \\
\hline & & & \\
\hline & 2.47343900 & -0.68577100 & \\
\hline & & & \\
\hline & & & \\
\hline & & & \\
\hline & & & \\
\hline & & & \\
\hline & & & \\
\hline & & & \\
\hline & & & \\
\hline & & & \\
\hline & & & \\
\hline & & & \\
\hline & & & \\
\hline & -1.252 & & \\
\hline \multicolumn{3}{|c|}{ Zero-point correction $=$} & \\
\hline \multicolumn{3}{|c|}{ Thermal correction to Energy= } & \\
\hline \multicolumn{4}{|c|}{ Thermal correction to Enthalpy $=$} \\
\hline \multicolumn{4}{|c|}{ Thermal correction to Gibbs Free Energy= } \\
\hline \multicolumn{4}{|c|}{ Sum of electronic and zero-point Energies $=$} \\
\hline \multicolumn{4}{|c|}{ um of electronic and thermal Energies $=$} \\
\hline \multirow{2}{*}{\multicolumn{4}{|c|}{$\begin{array}{l}\text { Sum of electronic and thermal Enthalpies= } \\
\text { Sum of electronic and thermal Free Energies= }\end{array}$}} \\
\hline & & & \\
\hline \multicolumn{4}{|c|}{ О-НЕН } \\
\hline & -3.4817 & & \\
\hline & & & \\
\hline & & & \\
\hline & & & \\
\hline & & & \\
\hline & & & \\
\hline & -3.5 & & \\
\hline & & & \\
\hline & & & \\
\hline & & & \\
\hline & & & \\
\hline & -2.29 & & \\
\hline & & & \\
\hline & & & \\
\hline & & & \\
\hline & 1.21563300 & 0.04389800 & -0.0194980 \\
\hline & & & \\
\hline & & & \\
\hline
\end{tabular}




\begin{tabular}{lrrr} 
H & -3.01603400 & -2.01594000 & -0.89225800 \\
$\mathrm{H}$ & -2.07979000 & -3.26688900 & -0.02539200 \\
$\mathrm{H}$ & -3.01030900 & -2.02470900 & 0.86021000 \\
$\mathrm{H}$ & -4.07050600 & 2.68682500 & 0.89782500 \\
$\mathrm{H}$ & -4.07682000 & 2.69629200 & -0.87368900 \\
$\mathrm{H}$ & 3.01855900 & -2.04521000 & 0.83937100 \\
$\mathrm{H}$ & 2.07346300 & -3.28101900 & -0.03966200 \\
$\mathrm{H}$ & 3.01213300 & -2.03645500 & -0.91310300 \\
$\mathrm{H}$ & -2.43803800 & 4.60069500 & 0.90538900 \\
$\mathrm{H}$ & -2.44431800 & 4.61013600 & -0.87246700 \\
$\mathrm{H}$ & -3.91588100 & 5.03555000 & 0.02395000 \\
$\mathrm{H}$ & 4.10501900 & 2.66821900 & -0.90120800 \\
$\mathrm{H}$ & 4.11115100 & 2.65918500 & 0.87031900 \\
$\mathrm{H}$ & 2.48574700 & 4.59320400 & -0.88898200 \\
$\mathrm{H}$ & 2.49173200 & 4.58404800 & 0.88888300 \\
$\mathrm{H}$ & 3.96635100 & 5.00871900 & -0.00285400 \\
$\mathrm{H}$ & 0.01426000 & 1.82170100 & -0.00597800 \\
Zero-point correction= & \multicolumn{4}{c}{0.290470} & $($ Hartree $/$ Particle $)$ \\
Thermal correction to Energy= & 0.309385 \\
Thermal correction to Enthalpy= & 0.310329 \\
Thermal correction to Gibbs Free Energy= & 0.240571 \\
Sum of electronic and zero-point Energies $=$ & -860.677980 \\
Sum of electronic and thermal Energies= & -860.659065 \\
Sum of electronic and thermal Enthalpies $=$ & -860.658121 \\
Sum of electronic and thermal Free Energies $=$ & -860.727879
\end{tabular}

$\begin{array}{lccc}\text { QNL } \\ \text { N } & 2.83066000 & -0.73292500 & 0.10975200 \\ \mathrm{C} & 3.16531300 & -1.95749200 & 0.44507700 \\ \mathrm{C} & 4.50087800 & -2.40058500 & 0.62294600 \\ \mathrm{C} & 5.51919600 & -1.50475700 & 0.43630800 \\ \mathrm{C} & 5.20847300 & -0.16906000 & 0.07313000 \\ \mathrm{C} & 6.20063200 & 0.82371400 & -0.14004700 \\ \mathrm{C} & 5.83961600 & 2.10024900 & -0.49023000 \\ \mathrm{C} & 4.47164400 & 2.44052700 & -0.64270600 \\ \mathrm{C} & 3.49203800 & 1.50067900 & -0.44276400 \\ \mathrm{C} & 3.83649400 & 0.17193100 & -0.07974000 \\ \mathrm{H} & 2.34722000 & -2.66079400 & 0.59077900 \\ \mathrm{H} & 4.69283100 & -3.43123100 & 0.90094400 \\ \mathrm{H} & 6.55963500 & -1.79485000 & 0.56036100 \\ \mathrm{H} & 7.24625800 & 0.55172700 & -0.02041600 \\ \mathrm{H} & 6.60046200 & 2.85741100 & -0.65254600 \\ \mathrm{H} & 4.20216700 & 3.45509000 & -0.92026000 \\ \mathrm{H} & 2.43905600 & 1.74094700 & -0.55453800 \\ \text { Zero-point correction= } & 0.137474 \text { (Hartree/Particle) } \\ \text { Thermal correction to Energy= } & 0.144096\end{array}$


Thermal correction to Enthalpy=

Thermal correction to Gibbs Free Energy=

Sum of electronic and zero-point Energies=

Sum of electronic and thermal Energies=

Sum of electronic and thermal Enthalpies=

Sum of electronic and thermal Free Energies=
0.145040

0.106364

$-401.633536$

$-401.626914$

$-401.625970$

$-401.664645$

\begin{tabular}{lrrr}
\multicolumn{4}{l}{ UN-INT1AE } \\
C & -1.08776200 & -1.66677200 & -1.33362800 \\
$\mathrm{C}$ & -1.16406100 & -0.28375500 & -1.10867800 \\
$\mathrm{C}$ & 0.01667800 & 0.43280900 & -0.95904000 \\
$\mathrm{H}$ & -0.03840900 & 1.49237500 & -0.73683900 \\
$\mathrm{C}$ & 1.27439900 & -0.15320700 & -1.14265400 \\
$\mathrm{C}$ & 1.33168200 & -1.51992100 & -1.40912600 \\
$\mathrm{~N}$ & 0.15781200 & -2.18129600 & -1.48217300 \\
$\mathrm{C}$ & 2.56637200 & -2.32411900 & -1.65320600 \\
$\mathrm{H}$ & 3.31315600 & -2.10750400 & -0.88977500 \\
$\mathrm{H}$ & 2.99966100 & -2.04965600 & -2.61970100 \\
$\mathrm{H}$ & 2.33497300 & -3.39164500 & -1.65306300
\end{tabular}




\begin{tabular}{|c|c|c|c|}
\hline $\mathrm{C}$ & -2.22359300 & -2.60015500 & -1.59681600 \\
\hline $\mathrm{H}$ & -3.06609900 & -2.37027900 & -0.94917700 \\
\hline $\mathrm{H}$ & -1.90748600 & -3.63311900 & -1.43308300 \\
\hline $\mathrm{H}$ & -2.55811000 & -2.48647200 & -2.63428700 \\
\hline $\mathrm{C}$ & 2.51854200 & 0.65009100 & -1.00849600 \\
\hline $\mathrm{C}$ & -2.48391600 & 0.40907600 & -1.05027900 \\
\hline 0 & -3.52842100 & -0.07108300 & -1.43292400 \\
\hline 0 & 3.62995100 & 0.26026400 & -1.29629100 \\
\hline 0 & 2.26266700 & 1.87994900 & -0.55520400 \\
\hline 0 & -2.36437300 & 1.66391700 & -0.60635000 \\
\hline $\mathrm{C}$ & 3.41016000 & 2.72580900 & -0.35621600 \\
\hline $\mathrm{H}$ & 4.07647600 & 2.24143700 & 0.36407500 \\
\hline $\mathrm{H}$ & 3.94482000 & 2.82202800 & -1.30540600 \\
\hline $\mathrm{C}$ & -3.59330900 & 2.40038800 & -0.48450900 \\
\hline $\mathrm{H}$ & -4.27681500 & 1.82962100 & 0.15125500 \\
\hline $\mathrm{H}$ & -4.04638500 & 2.49467200 & -1.47582400 \\
\hline $\mathrm{C}$ & -3.25250500 & 3.74499100 & 0.11419800 \\
\hline $\mathrm{H}$ & -2.81004700 & 3.62556300 & 1.10736000 \\
\hline $\mathrm{H}$ & -4.16224400 & 4.34412200 & 0.21022800 \\
\hline $\mathrm{H}$ & -2.54697000 & 4.28581600 & -0.52294400 \\
\hline $\mathrm{C}$ & 2.90319100 & 4.05693600 & 0.14737800 \\
\hline $\mathrm{H}$ & 2.36639300 & 3.93534500 & 1.09233500 \\
\hline $\mathrm{H}$ & 2.23065700 & 4.51661500 & -0.58223500 \\
\hline $\mathrm{H}$ & 3.74819300 & 4.73093200 & 0.31287600 \\
\hline $\mathrm{H}$ & -3.40394100 & 0.27553500 & 1.99918000 \\
\hline $\mathrm{C}$ & -2.43996900 & -0.21945500 & 1.90475600 \\
\hline $\mathrm{C}$ & -1.19845000 & 0.53727800 & 2.28855900 \\
\hline $\mathrm{N}$ & -1.27896600 & -2.29682100 & 1.32817900 \\
\hline $\mathrm{C}$ & 0.03354600 & -0.31723300 & 2.03959300 \\
\hline $\mathrm{C}$ & -2.39046500 & -1.51390800 & 1.50684300 \\
\hline $\mathrm{C}$ & -0.09700400 & -1.68257800 & 1.63321300 \\
\hline $\mathrm{C}$ & 1.30167400 & 0.23120700 & 2.20317800 \\
\hline $\mathrm{H}$ & -3.33366500 & -2.01352000 & 1.27196000 \\
\hline $\mathrm{H}$ & 0.99922800 & -3.48443000 & 1.23039300 \\
\hline $\mathrm{C}$ & 2.46773900 & -0.51363000 & 2.01338000 \\
\hline $\mathrm{H}$ & 1.37896900 & 1.28205700 & 2.48233500 \\
\hline $\mathrm{H}$ & 3.44394400 & -0.05699700 & 2.14982700 \\
\hline $\mathrm{C}$ & 2.35120000 & -1.86922800 & 1.69051800 \\
\hline $\mathrm{H}$ & 3.24319800 & -2.48133400 & 1.57723600 \\
\hline $\mathrm{C}$ & 1.10020900 & -2.43466200 & 1.49992800 \\
\hline $\mathrm{H}$ & 0.21237500 & -3.18671600 & -1.64451600 \\
\hline $\mathrm{H}$ & -1.12461800 & 1.48017800 & 1.72109200 \\
\hline $\mathrm{H}$ & -1.23392400 & 0.84804500 & 3.34742600 \\
\hline \multicolumn{4}{|c|}{ Zero-point correction $=$} \\
\hline \multicolumn{3}{|c|}{ Thermal correction to Energy= } & 0.479458 \\
\hline \multicolumn{4}{|c|}{ Thermal correction to Enthalpy= } \\
\hline
\end{tabular}


Thermal correction to Gibbs Free Energy=

Sum of electronic and zero-point Energies=

Sum of electronic and thermal Energies=

Sum of electronic and thermal Enthalpies=

Sum of electronic and thermal Free Energies=
0.394539

$-1263.434891$

$-1263.407455$

$-1263.406510$

$-1263.492373$ 


\begin{tabular}{|c|c|c|c|c|}
\hline & -2.97265200 & 1.26672700 & 0.38641500 & \\
\hline C & 2.78667300 & 2.58061300 & 1.29494800 & \\
\hline $\mathrm{H}$ & 3.53076200 & 2.25621300 & 0.55932700 & \\
\hline $\mathrm{H}$ & 3.25018200 & 2.51274200 & 2.28485500 & \\
\hline $\mathrm{C}$ & -4.16020100 & 1.90799500 & -0.08078300 & \\
\hline $\mathrm{H}$ & -4.98384400 & 1.69491100 & 0.60870100 & \\
\hline $\mathrm{H}$ & -4.43589600 & 1.49776000 & -1.05826100 & \\
\hline $\mathrm{C}$ & -3.86702200 & 3.39018900 & -0.16079100 & \\
\hline $\mathrm{H}$ & -3.59151400 & 3.78332000 & 0.82197600 & \\
\hline $\mathrm{H}$ & -4.75061900 & 3.92872700 & -0.51517800 & \\
\hline $\mathrm{H}$ & -3.04187600 & 3.58169400 & -0.85389800 & \\
\hline $\mathrm{C}$ & 2.28968300 & 3.98062300 & 1.00731200 & \\
\hline $\mathrm{H}$ & 1.54811600 & 4.28729500 & 1.75067000 & \\
\hline $\mathrm{H}$ & 1.82587200 & 4.03089800 & 0.01718100 & \\
\hline $\mathrm{H}$ & 3.12335300 & 4.68819200 & 1.03630500 & \\
\hline \multicolumn{3}{|c|}{ Zero-point correction $=$} & 0.4520 & 63 (Hartree/Particle) \\
\hline \multicolumn{3}{|c|}{ Thermal correction to Energy= } & & 79526 \\
\hline \multicolumn{3}{|c|}{ Thermal correction to Enthalpy= } & & 80471 \\
\hline \multicolumn{4}{|c|}{ Thermal correction to Gibbs Free Energy= } & 0.393705 \\
\hline \multicolumn{4}{|c|}{ Sum of electronic and zero-point Energies $=$} & -1263.448003 \\
\hline \multicolumn{4}{|c|}{ Sum of electronic and thermal Energies= } & -1263.420539 \\
\hline \multicolumn{4}{|c|}{ Sum of electronic and thermal Enthalpies= } & -1263.419595 \\
\hline \multicolumn{5}{|c|}{ Sum of electronic and thermal Free Energies $=$} \\
\hline
\end{tabular}

\begin{tabular}{lrrr}
\multicolumn{4}{l}{ UN-INT2G } \\
$\mathrm{H}$ & 4.37277600 & 1.36161000 & 1.63368200 \\
$\mathrm{C}$ & 3.94881400 & 0.39294900 & 1.38439600 \\
$\mathrm{C}$ & 2.86656500 & -2.10162800 & 0.72546500 \\
$\mathrm{C}$ & 2.58000600 & 0.19434200 & 1.53091200 \\
$\mathrm{C}$ & 4.77123300 & -0.63689800 & 0.92420800 \\
$\mathrm{C}$ & 4.23510200 & -1.88097800 & 0.59743000 \\
$\mathrm{C}$ & 2.07167000 & -1.06222500 & 1.19099800 \\
$\mathrm{H}$ & 5.83753900 & -0.46374500 & 0.81911700 \\
$\mathrm{H}$ & 4.87682500 & -2.67754000 & 0.23615700 \\
$\mathrm{H}$ & 2.41585600 & -3.05443500 & 0.46119800 \\
$\mathrm{~N}$ & 0.66712500 & -1.27104000 & 1.31888100 \\
$\mathrm{C}$ & -0.10329500 & -0.53124600 & 2.03611300 \\
$\mathrm{H}$ & -1.15650600 & -0.79739500 & 2.06350800 \\
$\mathrm{C}$ & 0.46350800 & 0.62444600 & 2.78197100 \\
$\mathrm{C}$ & 1.60905700 & 1.25635100 & 1.98454500 \\
$\mathrm{H}$ & 1.19155000 & 1.76362600 & 1.10319000 \\
$\mathrm{H}$ & 2.11739000 & 2.00902200 & 2.59123200 \\
$\mathrm{H}$ & -0.33370900 & 1.33923300 & 2.99250800 \\
$\mathrm{H}$ & 0.82361200 & 0.23699900 & 3.74740000 \\
$\mathrm{C}$ & -1.40017300 & -2.16241600 & -0.94663600 \\
$\mathrm{C}$ & 0.12093500 & 0.12009500 & -1.40589200
\end{tabular}




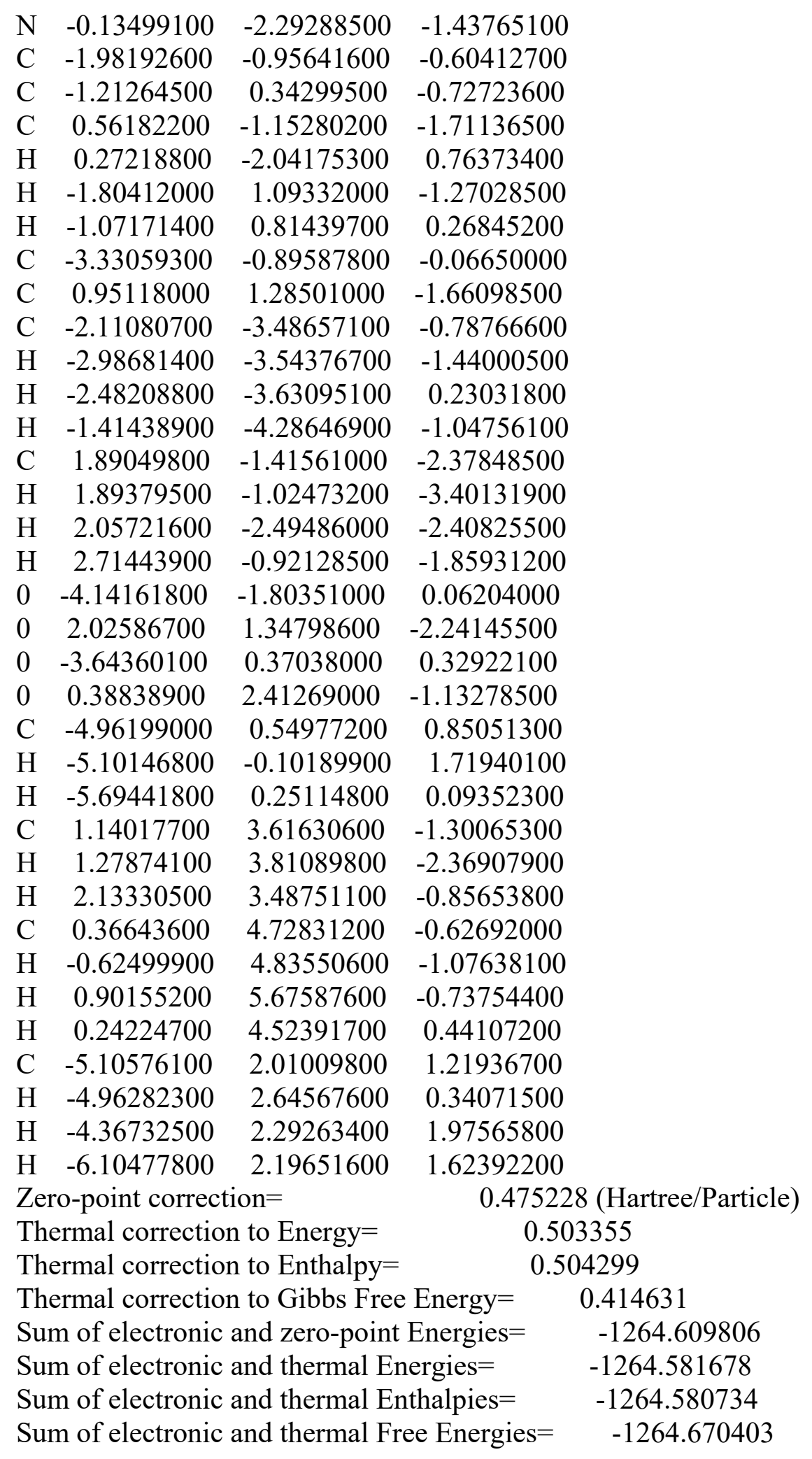

UN-TS1AE

$\begin{array}{llll}\text { C } & -2.20618600 & 3.59736400 & 0.41421000\end{array}$ 


\begin{tabular}{|c|c|c|c|}
\hline & & & \\
\hline & -2.39653500 & -1.32823000 & \\
\hline & 2.09159400 & & \\
\hline & -1.56055100 & & -0.48158500 \\
\hline & & & \\
\hline & -0.37249500 & -2.8 & \\
\hline & -2.75358 & & \\
\hline & 4.00927 & -0.77912 & \\
\hline & & & \\
\hline & -1.681 & & \\
\hline & & & \\
\hline & -1.2695 & 2.5 & \\
\hline & 3.1 & & \\
\hline & -0.6 & & \\
\hline & -1.0 & & \\
\hline & & & \\
\hline & 1.2 & & \\
\hline & -0.0 & & \\
\hline & & & \\
\hline & 0.9 & & \\
\hline & -2.47 & & \\
\hline & -2.57 & & \\
\hline & $-3.1^{\prime}$ & & \\
\hline & -2.4 & & \\
\hline & -3.1 & & \\
\hline & 2.5 & & \\
\hline & & -3.7 & \\
\hline & 2.92 & -2.8 & \\
\hline & -0.635 & & \\
\hline & -1.3 & & \\
\hline & -2.2 & & \\
\hline & & & \\
\hline & & & \\
\hline & & & 600 \\
\hline & & & \\
\hline & 5.304 & & 7400 \\
\hline & -0.309 & & \\
\hline & 0.908 & -2.4 & 9100 \\
\hline & 1.253 & & \\
\hline & 0.260 & & \\
\hline & -1.1244 & & 8600 \\
\hline & $-2.230^{\prime}$ & & 26900 \\
\hline & -3.5242 & -0.16093900 & 894300 \\
\hline & & & \\
\hline & -2.65289000 & -2.38337200 & -1.12935500 \\
\hline & -1.32337500 & -1.93697100 & -1.33208100 \\
\hline
\end{tabular}




\begin{tabular}{lrrr} 
H & 1.68830700 & -3.16758700 & -1.64422000 \\
$\mathrm{H}$ & 2.26869200 & -0.86093000 & -2.18051500 \\
$\mathrm{H}$ & 0.36140700 & 0.79656000 & -2.45132400 \\
$\mathrm{H}$ & -2.05425400 & 1.35277800 & -1.92260200 \\
$\mathrm{H}$ & -4.36755000 & 0.51964400 & -1.57833900 \\
$\mathrm{H}$ & -4.73630500 & -1.89550500 & -1.06169000 \\
$\mathrm{H}$ & -2.79856500 & -3.43846700 & -0.91050000 \\
Zero-point correction= & \multicolumn{3}{c}{0.448704 (Hartree/Particle) } \\
Thermal correction to Energy= & 0.475520 \\
Thermal correction to Enthalpy= & 0.476465 \\
Thermal correction to Gibbs Free Energy= & 0.390989 \\
Sum of electronic and zero-point Energies= & -1263.431109 \\
Sum of electronic and thermal Energies $=$ & -1263.404293 \\
Sum of electronic and thermal Enthalpies= & -1263.403349 \\
Sum of electronic and thermal Free Energies $=$ & -1263.488824
\end{tabular}

\begin{tabular}{lrcc}
\multicolumn{4}{l}{ UN-TS1BD } \\
H & 3.85744000 & -1.58099600 & -3.29470500 \\
$\mathrm{C}$ & 3.68804700 & -1.39748700 & -2.23812300 \\
$\mathrm{C}$ & 3.22082600 & -0.94001400 & 0.50825000 \\
$\mathrm{C}$ & 2.48494700 & -1.86698000 & -1.65106300 \\
$\mathrm{C}$ & 4.60872000 & -0.72648100 & -1.47481400 \\
$\mathrm{C}$ & 4.37299900 & -0.50024800 & -0.09557500 \\
$\mathrm{C}$ & 2.26713900 & -1.62309300 & -0.27345700 \\
$\mathrm{H}$ & 5.52794300 & -0.36481600 & -1.92315400 \\
$\mathrm{H}$ & 5.11650700 & 0.02787200 & 0.49251200 \\
$\mathrm{H}$ & 3.03318600 & -0.78442700 & 1.56588500 \\
$\mathrm{~N}$ & 1.09984800 & -2.06770700 & 0.28823500 \\
$\mathrm{C}$ & 0.17008200 & -2.72550200 & -0.38976300 \\
$\mathrm{H}$ & -0.71114900 & -3.02662700 & 0.16408100 \\
$\mathrm{C}$ & 0.34347400 & -2.99925900 & -1.75214100 \\
$\mathrm{C}$ & 1.49010000 & -2.56535500 & -2.37626800 \\
$\mathrm{H}$ & 1.64843800 & -2.75372700 & -3.43447400 \\
$\mathrm{H}$ & -0.42986000 & -3.53794200 & -2.28478700 \\
$\mathrm{C}$ & 0.49007500 & 0.50558100 & 2.16689000 \\
$\mathrm{C}$ & -1.82802800 & -0.34844300 & 0.89737200 \\
$\mathrm{~N}$ & -0.06818200 & -0.69665200 & 2.52521900 \\
$\mathrm{C}$ & 0.04715600 & 1.27055800 & 1.11429500 \\
$\mathrm{C}$ & -1.04887600 & 0.75241100 & 0.20435700 \\
$\mathrm{C}$ & -1.27524900 & -1.01847600 & 1.96660000 \\
$\mathrm{H}$ & 0.89274200 & -1.80176000 & 1.27845700 \\
$\mathrm{H}$ & -1.72144500 & 1.56989600 & -0.07425500 \\
$\mathrm{H}$ & -0.63362600 & 0.39974900 & -0.75874400 \\
$\mathrm{C}$ & 0.65096700 & 2.56006700 & 0.81004800 \\
$\mathrm{C}$ & -3.11763300 & -0.73755700 & 0.35021300 \\
$\mathrm{C}$ & 1.63335000 & 0.91041800 & 3.06700600
\end{tabular}




$\begin{array}{crcc}\mathrm{H} & 1.32307800 & 1.71775000 & 3.73924200 \\ \mathrm{H} & 2.49161700 & 1.28974400 & 2.50954700 \\ \mathrm{H} & 1.92085300 & 0.04799900 & 3.67394700 \\ \mathrm{C} & -1.93221200 & -2.20460300 & 2.63358600 \\ \mathrm{H} & -2.83536700 & -1.89639000 & 3.16977900 \\ \mathrm{H} & -1.22750200 & -2.63634800 & 3.34788900 \\ \mathrm{H} & -2.25563200 & -2.96300300 & 1.91613000 \\ 0 & 1.48894200 & 3.17342400 & 1.45678400 \\ 0 & -3.89393900 & -1.58419300 & 0.77366700 \\ 0 & 0.16903100 & 3.07031700 & -0.35517100 \\ 0 & -3.41241800 & -0.03336800 & -0.77651600 \\ \mathrm{C} & 0.68639100 & 4.34726500 & -0.73458400 \\ \mathrm{H} & 1.77274100 & 4.27729800 & -0.85296000 \\ \mathrm{H} & 0.48677100 & 5.07065500 & 0.06259500 \\ \mathrm{C} & -4.67023100 & -0.33322400 & -1.38467400 \\ \mathrm{H} & -5.47362700 & -0.15742900 & -0.66193100 \\ \mathrm{H} & -4.69792100 & -1.39344600 & -1.65743000 \\ \mathrm{C} & -4.80759300 & 0.55859900 & -2.59916800 \\ \mathrm{H} & -4.77764200 & 1.61280500 & -2.30896300 \\ \mathrm{H} & -5.76034900 & 0.36352600 & -3.09989200 \\ \mathrm{H} & -3.99732300 & 0.37146600 & -3.30991700 \\ \mathrm{C} & 0.00862400 & 4.74149200 & -2.02858400 \\ \mathrm{H} & -1.07483100 & 4.80591700 & -1.89241500 \\ \mathrm{H} & 0.21667300 & 4.00799200 & -2.81303700 \\ \mathrm{H} & 0.37524300 & 5.71731700 & -2.36010600 \\ \mathrm{Z} & -\mathrm{p} 0196017 & \end{array}$

Zero-point correction $=\quad 0.452041$ (Hartree/Particle)

Thermal correction to Energy= $\quad 0.478887$

Thermal correction to Enthalpy= $\quad 0.479831$

Thermal correction to Gibbs Free Energy $=0.393041$

Sum of electronic and zero-point Energies $=\quad-1263.442740$

Sum of electronic and thermal Energies $=\quad-1263.415895$

Sum of electronic and thermal Enthalpies $=\quad-1263.414951$

Sum of electronic and thermal Free Energies $=\quad-1263.501740$

$l l$
UN-TS1C
$\begin{array}{lrrr}\mathrm{C} & 2.58192600 & -3.76516800 & 0.02585700 \\ \mathrm{C} & 3.45086000 & 3.19282900 & 0.45085900 \\ \mathrm{C} & -1.95272400 & -1.96492300 & -1.87863000 \\ \mathrm{C} & -1.33448200 & 2.79988200 & -1.58440500 \\ \mathrm{C} & 3.83798000 & -3.52095800 & 0.82938900 \\ \mathrm{C} & 4.54777000 & 2.57243900 & 1.28392000 \\ 0 & 0.31659300 & -3.51006200 & -1.32269100 \\ 0 & 1.28279300 & 3.63175600 & -1.00902200 \\ \mathrm{C} & 1.18209900 & 0.00487700 & -0.85105500 \\ \mathrm{C} & 0.86565700 & -2.49026300 & -0.96215300 \\ \mathrm{C} & 1.51751900 & 2.48645000 & -0.69031500\end{array}$




\begin{tabular}{|c|c|c|c|}
\hline 0 & 2.02144400 & -2.47712400 & -0.28730800 \\
\hline 0 & 2.57581800 & 2.12509300 & 0.03877000 \\
\hline $\mathrm{C}$ & 0.36506700 & -1.11183900 & -1.18498800 \\
\hline $\mathrm{C}$ & -0.94348500 & -0.90617500 & -1.57142200 \\
\hline $\mathrm{C}$ & 0.68066400 & 1.30772100 & -1.04275500 \\
\hline $\mathrm{C}$ & -0.63941500 & 1.48897200 & -1.41158000 \\
\hline $\mathrm{N}$ & -1.38562300 & 0.37835600 & -1.65682500 \\
\hline $\mathrm{H}$ & 0.96829200 & -0.00071600 & 0.89671900 \\
\hline $\mathrm{H}$ & -2.92754900 & -1.51633100 & -2.08203700 \\
\hline $\mathrm{H}$ & -1.63401000 & -2.55455700 & -2.74122000 \\
\hline $\mathrm{H}$ & -2.04023900 & -2.65103600 & -1.03241600 \\
\hline $\mathrm{H}$ & 2.78772900 & -4.29483400 & -0.90892900 \\
\hline $\mathrm{H}$ & 1.84042000 & -4.34018200 & 0.58817200 \\
\hline $\mathrm{H}$ & -0.93454000 & 3.32506600 & -2.45603200 \\
\hline $\mathrm{H}$ & -2.40770600 & 2.64726400 & -1.71318200 \\
\hline $\mathrm{H}$ & -1.15991300 & 3.43282500 & -0.71266600 \\
\hline $\mathrm{H}$ & 4.55993600 & -2.93621800 & 0.25246000 \\
\hline $\mathrm{H}$ & 3.60713000 & -2.98158100 & 1.75251500 \\
\hline $\mathrm{H}$ & 4.29733600 & -4.47788100 & 1.09192000 \\
\hline $\mathrm{H}$ & 2.86557200 & 3.92097400 & 1.01902100 \\
\hline $\mathrm{H}$ & 3.84147900 & 3.68841200 & -0.44271000 \\
\hline $\mathrm{H}$ & 4.12866400 & 2.07331200 & 2.16206000 \\
\hline $\mathrm{H}$ & 5.11282300 & 1.84105200 & 0.69928400 \\
\hline $\mathrm{H}$ & 5.23533400 & 3.35229500 & 1.62262900 \\
\hline $\mathrm{N}$ & -0.72314500 & 1.03857700 & 1.61314000 \\
\hline $\mathrm{C}$ & 0.23669200 & 0.01378900 & 1.81466700 \\
\hline $\mathrm{C}$ & -0.24164200 & -1.39245100 & 1.92630900 \\
\hline $\mathrm{C}$ & -1.51596100 & -1.71394500 & 1.63138100 \\
\hline $\mathrm{C}$ & -2.45999000 & -0.67820700 & 1.27808900 \\
\hline $\mathrm{C}$ & -3.78908300 & -0.96170200 & 0.93642000 \\
\hline $\mathrm{C}$ & -4.69584300 & 0.04465700 & 0.63394900 \\
\hline $\mathrm{C}$ & -4.25562700 & 1.38047100 & 0.69840400 \\
\hline $\mathrm{C}$ & -2.95247800 & 1.68972600 & 1.03518400 \\
\hline $\mathrm{C}$ & -1.98201800 & 0.67816100 & 1.32372100 \\
\hline $\mathrm{H}$ & 0.94126000 & 0.27592900 & 2.61913100 \\
\hline $\mathrm{H}$ & 0.47818200 & -2.15552500 & 2.21622600 \\
\hline $\mathrm{H}$ & -1.86412200 & -2.74486000 & 1.68523000 \\
\hline $\mathrm{H}$ & -4.10235500 & -2.00513600 & 0.91419600 \\
\hline $\mathrm{H}$ & -5.72236800 & -0.18978500 & 0.37138100 \\
\hline $\mathrm{H}$ & -4.95363400 & 2.18684900 & 0.48393800 \\
\hline $\mathrm{H}$ & -2.62665400 & 2.72514300 & 1.09425400 \\
\hline $\mathrm{H}$ & 2.24085800 & -0.14190000 & -0.67955200 \\
\hline $\mathrm{H}$ & -2.36464800 & 0.51952400 & -1.89765000 \\
\hline \multicolumn{4}{|c|}{ Zero-point correction $=$} \\
\hline \multicolumn{3}{|c|}{ Thermal correction to Energy= } & 0.476631 \\
\hline \multicolumn{4}{|c|}{ Thermal correction to Enthalpy= } \\
\hline
\end{tabular}


Thermal correction to Gibbs Free Energy=

Sum of electronic and zero-point Energies $=$

Sum of electronic and thermal Energies=

Sum of electronic and thermal Enthalpies=

Sum of electronic and thermal Free Energies=
0.392114

$-1263.417352$

$-1263.390514$

$-1263.389570$

$-1263.475031$ 


\begin{tabular}{llll} 
H & -2.78732300 & 3.57202700 & -0.86037400 \\
$\mathrm{H}$ & -4.50942300 & 3.68823800 & -1.27964000 \\
$\mathrm{H}$ & -3.34622100 & 3.07223800 & -2.47086300 \\
$\mathrm{C}$ & 2.7361100 & 3.64684500 & -1.28048600 \\
$\mathrm{H}$ & 2.18158100 & 3.81687900 & -0.35305500 \\
$\mathrm{H}$ & 2.07144400 & 3.84719600 & -2.12565500 \\
$\mathrm{H}$ & 3.57171500 & 4.35127000 & -1.32091700 \\
$\mathrm{C}$ & 2.49428000 & -2.90902500 & -0.92493800 \\
$\mathrm{H}$ & 3.19909100 & -2.45524300 & -0.22437600 \\
$\mathrm{H}$ & 3.00435200 & -2.95866800 & -1.89200100 \\
$\mathrm{H}$ & 2.24748700 & -3.92436800 & -0.60634400 \\
$\mathrm{C}$ & -2.27394500 & -3.28625300 & -0.68090100 \\
$\mathrm{H}$ & -2.54599200 & -3.35277600 & 0.38093200 \\
$\mathrm{H}$ & -1.94429100 & -4.27257200 & -1.01288300 \\
$\mathrm{H}$ & -3.17212100 & -2.98554000 & -1.22109900 \\
Zero-point correction= & \multicolumn{4}{c}{0.447162} & (Hartree/Particle) \\
Thermal correction to Energy= & 0.473767 \\
Thermal correction to Enthalpy= & 0.474711 \\
Thermal correction to Gibbs Free Energy $=$ & 0.389942 \\
Sum of electronic and zero-point Energies $=$ & -1263.403143 \\
Sum of electronic and thermal Energies $=$ & -1263.376538 \\
Sum of electronic and thermal Enthalpies= & -1263.375594 \\
Sum of electronic and thermal Free Energies $=$ & -1263.460364
\end{tabular}

$\begin{array}{lrrr}\text { UN-TS2A } & & \\ \mathrm{C} & -0.39924400 & -1.72127900 & -1.14249600 \\ \mathrm{C} & -1.51223400 & -1.03290200 & -0.65139000 \\ \mathrm{C} & -1.42696900 & 0.34670600 & -0.48275600 \\ \mathrm{H} & -2.27750600 & 0.88593500 & -0.08309000 \\ \mathrm{C} & -0.28859500 & 1.05587700 & -0.86682600 \\ \mathrm{C} & 0.81452900 & 0.34808700 & -1.34804500 \\ \mathrm{~N} & 0.71111500 & -0.99997900 & -1.41916000 \\ \mathrm{C} & 2.08404800 & 0.95156800 & -1.85232900 \\ \mathrm{H} & 2.58269600 & 1.52199100 & -1.06402200 \\ \mathrm{H} & 1.85185400 & 1.64757100 & -2.66297100 \\ \mathrm{H} & 2.74969200 & 0.17010300 & -2.21958500 \\ \mathrm{C} & -0.34755800 & -3.17431300 & -1.49124100 \\ \mathrm{H} & -0.68994400 & -3.79115800 & -0.66106100 \\ \mathrm{H} & 0.66677500 & -3.45686400 & -1.77785700 \\ \mathrm{H} & -1.02013900 & -3.35524600 & -2.33597800 \\ \mathrm{C} & -0.24579500 & 2.54513000 & -0.74120300 \\ \mathrm{C} & -2.77436500 & -1.76181400 & -0.31667200 \\ 0 & -2.99369900 & -2.91777700 & -0.59827900 \\ 0 & 0.68419400 & 3.23791200 & -1.08553700 \\ 0 & -1.36451500 & 3.02207300 & -0.19677900 \\ 0 & -3.63536600 & -0.97781000 & 0.32918000\end{array}$




\begin{tabular}{|c|c|c|c|c|}
\hline \multirow{2}{*}{$\begin{array}{l}\mathrm{C} \\
\mathrm{H}\end{array}$} & -1.42056900 & 4.45337000 & \multicolumn{2}{|l|}{-0.02119400} \\
\hline & -0.58755200 & 4.75550700 & 0.61946500 & \\
\hline $\mathrm{H}$ & -1.29301500 & 4.92746300 & -0.99817900 & \\
\hline $\mathrm{C}$ & -4.89512400 & -1.58471500 & 0.68777900 & \\
\hline $\mathrm{H}$ & -4.69195900 & -2.45172800 & 1.32216100 & \\
\hline $\mathrm{H}$ & -5.38396800 & -1.93318400 & -0.22622900 & \\
\hline $\mathrm{C}$ & -5.70864400 & -0.53291700 & 1.40391200 & \\
\hline $\mathrm{H}$ & -5.19394300 & -0.19321400 & 2.30678000 & \\
\hline $\mathrm{H}$ & -6.67447400 & -0.95576000 & 1.69353600 & \\
\hline $\mathrm{H}$ & -5.88782100 & 0.32818000 & 0.75413100 & \\
\hline $\mathrm{C}$ & -2.76071000 & 4.77392300 & 0.59717000 & \\
\hline $\mathrm{H}$ & -2.86895700 & 4.27856400 & 1.56593100 & \\
\hline $\mathrm{H}$ & -3.57617800 & 4.45256500 & -0.05668900 & \\
\hline $\mathrm{H}$ & -2.84241700 & 5.85367300 & 0.74869700 & \\
\hline $\mathrm{H}$ & 0.46752800 & -3.00744500 & 2.21060700 & \\
\hline $\mathrm{C}$ & 1.13130200 & -2.37995600 & 1.62204500 & \\
\hline $\mathrm{C}$ & 1.23737000 & -0.91141700 & 1.93996800 & \\
\hline $\mathrm{N}$ & 2.77262300 & -2.26500200 & -0.18853100 & \\
\hline $\mathrm{C}$ & 2.52734600 & -0.35054200 & 1.36549100 & \\
\hline $\mathrm{C}$ & 1.85651300 & -2.91593200 & 0.61444200 & \\
\hline $\mathrm{C}$ & 3.21396200 & -1.06332300 & 0.34804700 & \\
\hline $\mathrm{C}$ & 3.04105400 & 0.86565200 & 1.82133800 & \\
\hline $\mathrm{H}$ & 1.71298400 & -3.97055100 & 0.36921800 & \\
\hline $\mathrm{H}$ & 4.94886800 & -1.07010500 & -0.91132800 & \\
\hline $\mathrm{C}$ & 4.21313300 & 1.41078700 & 1.30220700 & \\
\hline $\mathrm{H}$ & 2.49937800 & 1.39836100 & 2.60216800 & \\
\hline $\mathrm{H}$ & 4.58786800 & 2.36165500 & 1.66898500 & \\
\hline $\mathrm{C}$ & 4.90617400 & 0.70254600 & 0.31368600 & \\
\hline $\mathrm{H}$ & 5.83243300 & 1.10026700 & -0.09343300 & \\
\hline $\mathrm{C}$ & 4.41710700 & -0.50956700 & -0.14562700 & \\
\hline $\mathrm{H}$ & 1.62671800 & -1.55742000 & -1.40936900 & \\
\hline $\mathrm{H}$ & 0.36586500 & -0.35668000 & 1.54124100 & \\
\hline $\mathrm{H}$ & 1.20776000 & -0.73260900 & 3.02417700 & \\
\hline \multicolumn{3}{|c|}{ Zero-point correction $=$} & 0.4503( & 7 (Hartree/Particle) \\
\hline \multicolumn{3}{|c|}{ Thermal correction to Energy= } & 0.47 & 7058 \\
\hline \multicolumn{3}{|c|}{ Thermal correction to Enthalpy= } & 0.4 & 78002 \\
\hline \multicolumn{4}{|c|}{ Thermal correction to Gibbs Free Energy= } & 0.391792 \\
\hline \multicolumn{4}{|c|}{ Sum of electronic and zero-point Energies $=$} & -1263.429729 \\
\hline \multicolumn{4}{|c|}{ Sum of electronic and thermal Energies= } & -1263.402979 \\
\hline \multicolumn{4}{|c|}{ Sum of electronic and thermal Enthalpies= } & -1263.402034 \\
\hline \multicolumn{4}{|c|}{ Sum of electronic and thermal Free Energies $=$} & -1263.488245 \\
\hline \multicolumn{5}{|c|}{ UN-TS2B } \\
\hline $\mathrm{C}$ & 2.55352400 & 3.10481000 & -0.81579200 & \\
\hline $\mathrm{C}$ & -4.27113600 & 1.71582100 & 0.28402000 & \\
\hline $\mathrm{C}$ & 2.20659500 & -1.94936500 & -2.09032300 & \\
\hline
\end{tabular}




\begin{tabular}{|c|c|c|c|}
\hline & & 91776900 & \\
\hline & & & \\
\hline & & & \\
\hline & 2.95453300 & & \\
\hline & -4.03038000 & & \\
\hline & -0.56 & & \\
\hline & & & \\
\hline & -3.00160000 & -0.07 & \\
\hline & & & \\
\hline & -3.00 & & \\
\hline & & & \\
\hline & 0.8 & -1.3 & \\
\hline & -1.6 & & \\
\hline & -1.35 & -1.8 & \\
\hline & -0.1 & -2.2 & \\
\hline & -0.4 & & \\
\hline & 2.1 & -3.0 & \\
\hline & & -1.6 & \\
\hline & 3.0 & -1.6 & \\
\hline & 2.8 & & \\
\hline & 3.40 & & \\
\hline & -3.1 & & \\
\hline & -1.9 & -3.8 & \\
\hline & -2.9 & -2.9 & \\
\hline & & & \\
\hline & 1.6 & 4.0 & \\
\hline & 2.74 & & \\
\hline & -4.68 & & \\
\hline & -4.9 & & \\
\hline & & & \\
\hline & -3.6 & & \\
\hline & -4.98 & & \\
\hline & -0.03 & -2.2 & \\
\hline & -1.22 & -1.70 & \\
\hline & -1.3 & -0.3 & \\
\hline & -0.23 & 0.4 & \\
\hline & 1.07 & -0.1 & \\
\hline & 2.26 & 0.59 & \\
\hline & 3.48721800 & -0.030903 & \\
\hline & 3.53 & -1.42 & \\
\hline & 2.375 & -2.17 & \\
\hline & 1.14 & -1.53 & \\
\hline & -2.05799400 & -2.39718800 & 27900 \\
\hline & -2.31856400 & & \\
\hline & -0.27753800 & 1.53244100 & 2.12169900 \\
\hline & 2.21361000 & 1.67560800 & 1.87157400 \\
\hline
\end{tabular}




\begin{tabular}{|c|c|c|c|}
\hline & 4.40428500 & 4915900 & \\
\hline & 4.49136800 & & \\
\hline & 2.40314200 & .25161400 & \\
\hline & 0.01993200 & & \\
\hline & -0.82537300 & 1.49060700 & 18765200 \\
\hline \multicolumn{3}{|c|}{ Zero-point correction $=$} & \\
\hline \multicolumn{3}{|c|}{ Thermal correction to Energy= } & \\
\hline \multicolumn{4}{|c|}{ Thermal correction to Enthalpy $=$} \\
\hline \multicolumn{4}{|c|}{ Thermal correction to Gibbs Free Energy= } \\
\hline \multicolumn{4}{|c|}{ Sum of electronic and zero-point Energies $=$} \\
\hline \multicolumn{4}{|c|}{ Sum of electronic and thermal Energies= } \\
\hline \multirow{2}{*}{\multicolumn{4}{|c|}{$\begin{array}{l}\text { Sum of electronic and thermal Enthalpies }= \\
\text { Sum of electronic and thermal Free Energies= }\end{array}$}} \\
\hline & & & \\
\hline \multicolumn{4}{|c|}{ UN-TS2D } \\
\hline & -2.39338300 & 0.45003100 & 21081080 \\
\hline & & & \\
\hline & -0.0 & & \\
\hline c & & & \\
\hline & -2.3 & & \\
\hline & 3.17 & & \\
\hline & $-1.7 \varepsilon$ & & \\
\hline & & & \\
\hline & & & \\
\hline & -0.9 & & \\
\hline & & & \\
\hline & -1.14 & & 1.5 \\
\hline & & & \\
\hline & 0.3 & & \\
\hline & 0.78 & & \\
\hline & & -0.6 & 9330 \\
\hline C & & & \\
\hline & & & \\
\hline & & & \\
\hline & & & \\
\hline $\mathrm{I}$ & -0.32 & & \\
\hline & & & \\
\hline & -2.4 & & \\
\hline & -3.2 & & \\
\hline & & & -0. \\
\hline $\mathrm{H}$ & & & -1.2425810 \\
\hline H & & & \\
\hline & & & \\
\hline & & 2.49221800 & 1.507841 \\
\hline $\mathrm{H}$ & -3.34458700 & 2.23677500 & 2.9089430 \\
\hline & 4.39758000 & 2.87952100 & -0.7640870 \\
\hline
\end{tabular}




\begin{tabular}{lrrr}
$\mathrm{H}$ & 4.78039000 & 2.57964400 & 0.93597000 \\
$\mathrm{H}$ & 2.36656100 & 4.19956900 & -0.07689200 \\
$\mathrm{H}$ & 2.74685000 & 3.88745200 & 1.62985000 \\
$\mathrm{H}$ & 3.83716500 & 4.88250000 & 0.64369000 \\
$\mathrm{~N}$ & -1.07670700 & -0.35699000 & -1.58411800 \\
$\mathrm{C}$ & -0.08944900 & 0.57497000 & -1.66729200 \\
$\mathrm{C}$ & -0.47515700 & 1.96149900 & -1.61578000 \\
$\mathrm{C}$ & -1.75172900 & 2.30440200 & -1.32390000 \\
$\mathrm{C}$ & -2.75542800 & 1.29313000 & -1.10461900 \\
$\mathrm{C}$ & -4.08872300 & 1.59679800 & -0.77876700 \\
$\mathrm{C}$ & -5.01959400 & 0.58807000 & -0.60502100 \\
$\mathrm{C}$ & -4.63323100 & -0.75408900 & -0.75072800 \\
$\mathrm{C}$ & -3.32542800 & -1.08485900 & -1.06090000 \\
$\mathrm{C}$ & -2.38530000 & -0.06112300 & -1.24248400 \\
$\mathrm{H}$ & 0.79331200 & 0.26818800 & -2.22559000 \\
$\mathrm{H}$ & 0.29506900 & 2.70475200 & -1.78604800 \\
$\mathrm{H}$ & -2.04738500 & 3.34820600 & -1.26426700 \\
$\mathrm{H}$ & -4.37224900 & 2.64023500 & -0.66934000 \\
$\mathrm{H}$ & -6.04737700 & 0.83121000 & -0.35628300 \\
$\mathrm{H}$ & -5.36337900 & -1.54495100 & -0.61121800 \\
$\mathrm{H}$ & -3.01640200 & -2.12077500 & -1.16269600 \\
$\mathrm{H}$ & -0.84164600 & -1.32893900 & -1.76270900 \\
$\mathrm{H}$ & 1.19652300 & 0.51386600 & 1.27443100 \\
Zero-point correction= & \multicolumn{3}{c}{0.448515 (Hartree/Particle) } \\
Thermal correction to Energy= & \multicolumn{2}{c}{0.475280} \\
Thermal correction to Enthalpy= & 0.476224 \\
Thermal correction to Gibbs Free Energy= & 0.391526 \\
Sum of electronic and zero-point Energies $=$ & -1263.432754 \\
Sum of electronic and thermal Energies= & -1263.405989 \\
Sum of electronic and thermal Enthalpies $=$ & -1263.405044 \\
Sum of electronic and thermal Free Energies $=$ & -1263.489742 \\
\multicolumn{5}{|c|}{}
\end{tabular}

UN-TS2E

$\begin{array}{lrrc}\mathrm{C} & 3.98762200 & -2.50403800 & 0.39095600 \\ \mathrm{C} & 1.70736200 & 4.11054500 & -0.42463400 \\ \mathrm{C} & -0.88879800 & -3.21650300 & -1.46094700 \\ \mathrm{C} & -2.48723400 & 1.28554900 & -1.86683200 \\ \mathrm{C} & 4.98703900 & -1.63277600 & 1.11456300 \\ \mathrm{C} & 3.00130500 & 4.20138600 & 0.34965100 \\ \mathrm{H} & -2.52891300 & -1.18417800 & -1.11330200 \\ 0 & 1.85789900 & -3.44674100 & -0.87832400 \\ 0 & -0.52824000 & 3.27571600 & -1.57713900 \\ \mathrm{C} & 0.97753700 & 0.05534400 & -0.84452700 \\ \mathrm{C} & 1.86239900 & -2.27041900 & -0.59333500 \\ \mathrm{C} & 0.22486400 & 2.43181100 & -1.14360600 \\ 0 & 2.86331000 & -1.66761900 & 0.05003100\end{array}$




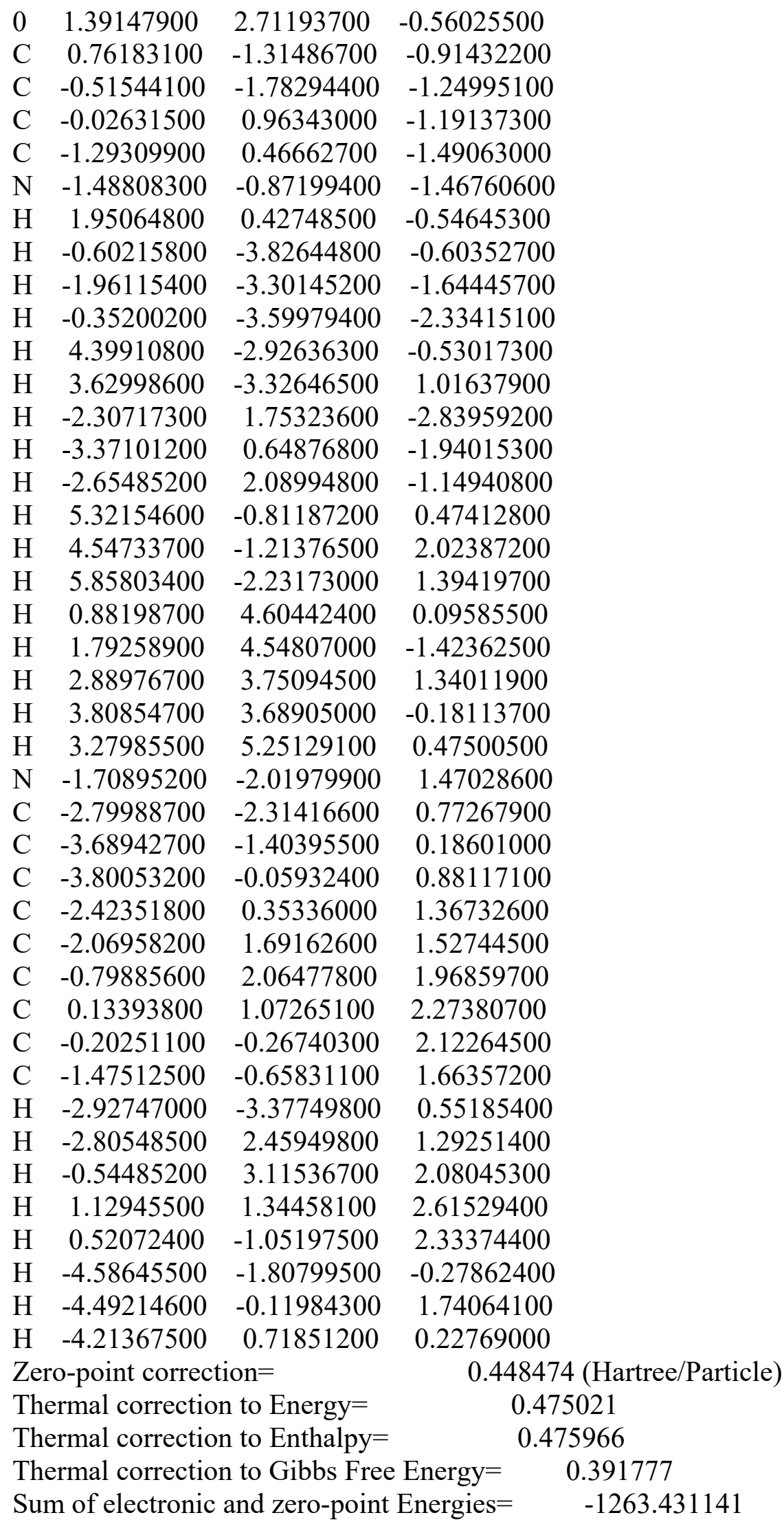




\begin{tabular}{|c|c|c|c|}
\hline \multicolumn{4}{|c|}{$\begin{array}{l}\text { Sum of electronic and thermal Energies }= \\
\text { Sum of electronic and thermal Enthalpies }= \\
\text { Sum of electronic and thermal Free Energies= }\end{array}$} \\
\hline \multicolumn{4}{|c|}{ UN-TS3G } \\
\hline & -0.37589400 & -0.96461200 & 2.48640900 \\
\hline & 0.48547900 & & 2.21545400 \\
\hline & 2.67635600 & & 1.47807500 \\
\hline & 0.46395700 & 1.02127300 & 2.422 \\
\hline & 1.59198800 & & \\
\hline & 2.68390200 & -0.1 & 1.29 \\
\hline & & & \\
\hline & -0.41178400 & & \\
\hline & & & \\
\hline & 3.5323 & 1.7 & 700 \\
\hline & & & \\
\hline & & & \\
\hline & & & \\
\hline & & & \\
\hline & 4.5 & -2.2 & -0.4 \\
\hline & & & \\
\hline & 4.62 & -0.2 & 0.5 \\
\hline & & & \\
\hline & 0.6539 & -2.8 & 1.2 \\
\hline & & & -1.6 \\
\hline & & & -1.22 \\
\hline & & -1.5 & 400 \\
\hline & & & -0.8 \\
\hline & -1.21293700 & & -0.69 \\
\hline & & & 0.3 \\
\hline & -1.9885 & 0.90 & -1.28 \\
\hline & -0.46691 & -1.9 & 9100 \\
\hline & -1.4436 & & 6700 \\
\hline & & & \\
\hline & & & -1.25 \\
\hline & -3.14812200 & -2.7 & -0.25 \\
\hline & 1.55967200 & & -1.712 \\
\hline & -3.54167900 & -0.57235600 & 0.13 \\
\hline & -0.40311500 & 2.92346600 & -0.65789800 \\
\hline & -4.85030200 & -0.94 & 0.57279200 \\
\hline & -4.76450100 & -1.64214100 & 1.41211900 \\
\hline & -5.36997300 & -1.46435000 & -0.23835800 \\
\hline & -0.10593800 & 4.31824500 & -0.57019700 \\
\hline & 0.86907000 & 4.45017100 & -0.08986200 \\
\hline & -0.03723900 & 4.73865500 & -1.57910100 \\
\hline & -5.56857300 & 0.32559100 & 0.97294300 \\
\hline
\end{tabular}




\begin{tabular}{|c|c|c|c|c|}
\hline 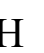 & -5.03551500 & 0.83270600 & 1.78241800 & \\
\hline $\mathrm{H}$ & -6.57937100 & 0.08916400 & 1.31766300 & \\
\hline $\mathrm{H}$ & -5.64284100 & 1.01070900 & 0.12350500 & \\
\hline $\mathrm{C}$ & -1.21538600 & 4.96278000 & 0.23126400 & \\
\hline $\mathrm{H}$ & -1.26620800 & 4.52790900 & 1.23421500 & \\
\hline $\mathrm{H}$ & -2.18209200 & 4.81666300 & -0.25911700 & \\
\hline $\mathrm{H}$ & -1.03306900 & 6.03703600 & 0.32671800 & \\
\hline $\mathrm{C}$ & 2.41511300 & 0.07028100 & -2.18138500 & \\
\hline $\mathrm{H}$ & 2.99809700 & 0.70114000 & -1.50609400 & \\
\hline $\mathrm{H}$ & 2.34197200 & 0.62124200 & -3.12370600 & \\
\hline $\mathrm{H}$ & 2.93972600 & -0.87071600 & -2.36904300 & \\
\hline $\mathrm{C}$ & -0.63314900 & -3.47421000 & -1.14185100 & \\
\hline $\mathrm{H}$ & -0.97505300 & -3.85907300 & $-0.1788080 c$ & \\
\hline $\mathrm{H}$ & 0.31826800 & -3.93773600 & -1.41522100 & \\
\hline $\mathrm{H}$ & -1.38967500 & -3.77175700 & $-1.8742750 c$ & \\
\hline \multicolumn{3}{|c|}{ Zero-point correction $=$} & 0.4707 & 4 (Hartree/Particle) \\
\hline \multicolumn{3}{|c|}{ Thermal correction to Energy= } & 0.4 & 8133 \\
\hline \multicolumn{3}{|c|}{ Thermal correction to Enthalpy= } & & 99077 \\
\hline \multicolumn{4}{|c|}{ Thermal correction to Gibbs Free Energy= } & 0.412272 \\
\hline \multicolumn{4}{|c|}{ Sum of electronic and zero-point Energies= } & -1264.605052 \\
\hline \multicolumn{4}{|c|}{ Sum of electronic and thermal Energies= } & -1264.577684 \\
\hline \multicolumn{4}{|c|}{ Sum of electronic and thermal Enthalpies $=$} & -1264.576740 \\
\hline \multicolumn{4}{|c|}{ Sum of electronic and thermal Free Energies= } & -1264.663544 \\
\hline
\end{tabular}

\begin{tabular}{llll}
\multicolumn{4}{l}{ UN-TS3H } \\
H & -1.58505100 & 1.75405300 & -0.03149600 \\
$\mathrm{C}$ & -1.40707300 & 0.68347200 & 0.05283600 \\
$\mathrm{C}$ & -0.94070400 & -2.04308200 & 0.26606500 \\
$\mathrm{C}$ & -0.08923600 & 0.23368800 & 0.10655000 \\
$\mathrm{C}$ & -2.48687100 & -0.19687500 & 0.10776200 \\
$\mathrm{C}$ & -2.24479000 & -1.56628600 & 0.22937200 \\
$\mathrm{C}$ & 0.13900500 & -1.15247200 & 0.19011600 \\
$\mathrm{H}$ & -3.50333200 & 0.18288200 & 0.06974700 \\
$\mathrm{H}$ & -3.07283600 & -2.26692800 & 0.28975900 \\
$\mathrm{H}$ & -0.74129100 & -3.10916600 & 0.34449600 \\
$\mathrm{~N}$ & 1.44524800 & -1.63314400 & 0.18648000 \\
$\mathrm{C}$ & 2.48905700 & -0.81292000 & -0.21633500 \\
$\mathrm{H}$ & 3.42942300 & -1.32538400 & -0.38927100 \\
$\mathrm{C}$ & 2.34381100 & 0.52922000 & -0.37528300 \\
$\mathrm{C}$ & 1.07770200 & 1.20279200 & 0.12060800 \\
$\mathrm{H}$ & 1.22928800 & 1.58305600 & 1.14187500 \\
$\mathrm{H}$ & 0.83622500 & 2.07474300 & -0.50063200 \\
$\mathrm{H}$ & 3.24446600 & 1.12932700 & -0.47337900 \\
$\mathrm{H}$ & 1.56118200 & -2.62905200 & 0.04025600 \\
$\mathrm{C}$ & 4.86625600 & -1.58679800 & -3.23730700 \\
$\mathrm{C}$ & 1.21102900 & 4.56885500 & -3.83200800
\end{tabular}




\begin{tabular}{|c|c|c|c|}
\hline $\mathrm{C}$ & -0.10542700 & -3.11587900 & -2.79176000 \\
\hline $\mathrm{C}$ & -2.55309200 & 1.00340100 & -3.20309400 \\
\hline $\mathrm{C}$ & 5.91236100 & -0.50268600 & -3.36920900 \\
\hline $\mathrm{C}$ & 2.66664500 & 4.97728300 & -3.80459300 \\
\hline $\mathrm{H}$ & -1.94910600 & -1.40217000 & -2.80652600 \\
\hline $\mathrm{O}$ & 2.64316500 & -2.95607500 & -2.95417900 \\
\hline $\mathrm{O}$ & -1.07010400 & 3.27030900 & -3.78231700 \\
\hline $\mathrm{C}$ & 1.32268200 & 0.48333800 & -3.31522000 \\
\hline $\mathrm{C}$ & 2.51746200 & -1.75509400 & -3.13995000 \\
\hline $\mathrm{C}$ & -0.06519900 & 2.59972100 & -3.61692300 \\
\hline $\mathrm{O}$ & 3.58631400 & -0.95190900 & -3.32218900 \\
\hline $\mathrm{O}$ & 1.16347100 & 3.15253000 & -3.61517900 \\
\hline $\mathrm{C}$ & 1.26337300 & -0.99599900 & -3.20696800 \\
\hline $\mathrm{C}$ & 0.07888100 & -1.64212900 & -3.01188800 \\
\hline $\mathrm{C}$ & -0.00150700 & 1.14701700 & -3.40004700 \\
\hline $\mathrm{C}$ & -1.15655300 & 0.45060300 & -3.20901500 \\
\hline $\mathrm{N}$ & -1.08765700 & -0.91238000 & -3.01104100 \\
\hline $\mathrm{H}$ & 2.03184000 & 0.80994800 & -1.92119200 \\
\hline $\mathrm{H}$ & 2.05691300 & 0.85593900 & -4.02678600 \\
\hline $\mathrm{H}$ & 0.43988700 & -3.43985300 & -1.90180000 \\
\hline $\mathrm{H}$ & -1.16547200 & -3.35618500 & -2.67167800 \\
\hline $\mathrm{H}$ & 0.29903500 & -3.68649700 & -3.63077200 \\
\hline $\mathrm{H}$ & 4.95046900 & -2.33401500 & -4.03306000 \\
\hline $\mathrm{H}$ & 4.94484000 & -2.11123600 & -2.27932200 \\
\hline $\mathrm{H}$ & -2.83834000 & 1.34261900 & -4.20271500 \\
\hline $\mathrm{H}$ & -3.26137700 & 0.23751500 & -2.87487700 \\
\hline $\mathrm{H}$ & -2.62864700 & 1.86718700 & -2.54107500 \\
\hline $\mathrm{H}$ & 5.81605100 & 0.01331300 & -4.32874500 \\
\hline $\mathrm{H}$ & 5.80607300 & 0.23315100 & -2.56654400 \\
\hline $\mathrm{H}$ & 6.91263000 & -0.94089800 & -3.30927900 \\
\hline $\mathrm{H}$ & 0.63364500 & 5.06918600 & -3.04837500 \\
\hline $\mathrm{H}$ & 0.74121000 & 4.79930100 & -4.79324200 \\
\hline $\mathrm{H}$ & 3.11845100 & 4.73100300 & -2.83943100 \\
\hline $\mathrm{H}$ & 3.22650000 & 4.46509600 & -4.59229700 \\
\hline $\mathrm{H}$ & 2.75183000 & 6.05603800 & -3.96324600 \\
\hline \multicolumn{3}{|c|}{ Zero-point correction $=$} & 0.469672 (Hartree/Particle) \\
\hline \multicolumn{3}{|c|}{ Thermal correction to Energy= } & 0.497581 \\
\hline \multicolumn{3}{|c|}{ Thermal correction to Enthalpy $=$} & 0.498525 \\
\hline \multicolumn{4}{|c|}{ Thermal correction to Gibbs Free Energy $=0.410^{\prime}$} \\
\hline \multicolumn{4}{|c|}{ Sum of electronic and zero-point Energies $=$} \\
\hline \multicolumn{4}{|c|}{ Sum of electronic and thermal Energies $=$} \\
\hline \multicolumn{4}{|c|}{ Sum of electronic and thermal Enthalpies $=$} \\
\hline \multicolumn{4}{|c|}{ Sum of electronic and thermal Free Energies $=$} \\
\hline
\end{tabular}

\section{UN-TS4G}

$\begin{array}{llll}\text { C } & 4.89818500 & 0.28803100 & 0.81888700\end{array}$ 


\begin{tabular}{|c|c|c|c|}
\hline & & & \\
\hline & & & \\
\hline & & & \\
\hline & 5.04101400 & & \\
\hline & -0.14174300 & & \\
\hline & 4.04 & -1.8 & \\
\hline & -2.13 & & \\
\hline & 1.0 & & \\
\hline & & & \\
\hline & -1.0 & & \\
\hline & & & \\
\hline & -0.3 & & -0.7 \\
\hline & & & \\
\hline & 1.2 & -2. & \\
\hline & -0.2 & & -1.2 \\
\hline & -0.7 & & \\
\hline & -0.0 & & -1.7 \\
\hline & & & \\
\hline & 1.6 & & -0.9 \\
\hline & 2.3 & & \\
\hline & & $-4 .($ & \\
\hline & & & \\
\hline & & & \\
\hline & 5.0 & -0 . & \\
\hline & -2.2 & & -3. \\
\hline & -2.3 & -2 . & -2 \\
\hline & -2.9 & & -1.5 \\
\hline & & & \\
\hline & 4.3 & & \\
\hline & 6.0 & & \\
\hline & -2.0 & & \\
\hline & & & \\
\hline & -0.0 & & \\
\hline & & & \\
\hline & -0.5 & & \\
\hline & -1.3 & & 100 \\
\hline & -0.1 & & \\
\hline & 0.1 & -0.5 & 2.54724200 \\
\hline & -0.5 & & \\
\hline & & & \\
\hline & -2.96 & & 1.24360900 \\
\hline & -4.2 & -1.9 & 0.88523700 \\
\hline & -4.58 & -0.60 & 0.71153700 \\
\hline & -3.60 & & \\
\hline & -2.32631800 & -0.02183200 & 1.25970000 \\
\hline & -2.71599300 & -3.36877200 & 1.36923800 \\
\hline
\end{tabular}




\begin{tabular}{lrrc} 
H & -5.02059900 & -2.70746900 & 0.73826300 \\
$\mathrm{H}$ & -5.58972400 & -0.30799100 & 0.42831900 \\
$\mathrm{H}$ & -3.83121200 & 1.42973500 & 0.75242200 \\
$\mathrm{H}$ & -1.55858500 & 1.92103300 & 1.16549600 \\
$\mathrm{H}$ & 0.44686900 & 1.62976500 & 2.20714000 \\
$\mathrm{H}$ & -0.17650200 & -0.58284900 & 3.59685500 \\
$\mathrm{H}$ & 1.24023900 & -0.72471900 & 2.55488100 \\
$\mathrm{H}$ & -0.49435000 & -2.63995600 & 2.32901200 \\
$\mathrm{H}$ & -0.00884000 & -1.87384200 & 0.81975200 \\
Zero-point correction= & 0.473673 (Hartree/Particle) \\
Thermal correction to Energy= & \multicolumn{2}{c}{0.501094} \\
Thermal correction to Enthalpy= & 0.502038 \\
Thermal correction to Gibbs Free Energy= & 0.415320 \\
Sum of electronic and zero-point Energies= & -1264.608907 \\
Sum of electronic and thermal Energies $=$ & -1264.581486 \\
Sum of electronic and thermal Enthalpies= & -1264.580542 \\
Sum of electronic and thermal Free Energies= & -1264.667259
\end{tabular}

